$b+\div 86739$

17760604

\title{
A FATIGUE DAMAGE MODEL DEVELOPED BASED ON STIFFNESS DEGRADATION IN HUMAN HAVERSIAN CORTICAL BONE
}

\author{
by \\ Hossein Najmi \\ B.Sc. in Mechanical Engineering, \\ Sharif University of Technology, Tehran, Iran, 1996

\begin{abstract}
A thesis
presented to Ryerson University

in partial fulfilment of the

requirements for the degree of

Master of Applied Science

in the program of

Mechanical Engineering
\end{abstract}

Toronto, Ontario, Canada, 2007

(C) Hossein Najmi 
UMI Number: EC53676

\section{INFORMATION TO USERS}

The quality of this reproduction is dependent upon the quality of the copy submitted. Broken or indistinct print, colored or poor quality illustrations and photographs, print bleed-through, substandard margins, and improper alignment can adversely affect reproduction.

In the unlikely event that the author did not send a complete manuscript and there are missing pages, these will be noted. Also, if unauthorized copyright material had to be removed, a note will indicate the deletion.

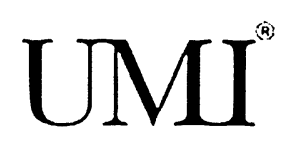

UMI Microform EC53676

Copyright 2009 by ProQuest LLC

All rights reserved. This microform edition is protected against unauthorized copying under Title 17, United States Code.

ProQuest LLC

789 East Eisenhower Parkway

P.O. Box 1346

Ann Arbor, MI 48106-1346 


\section{Author's Declaration}

I hereby declare that I am the sole author of this thesis.

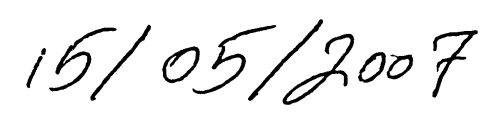

1

I authorize the Ryerson University to lend this thesis to other institutions or individuals for the purpose of scholarly research.
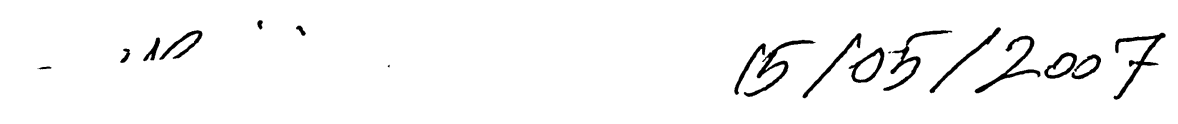

I further authorize the Ryerson University to reproduce this thesis by photocopying or by other means, in total or in part, at the request of other institutions or individuals for the purpose of scholarly research.

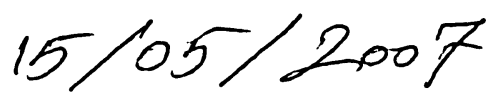


Ryerson University requires the signatures of all persons using or photocopying this thesis. Please sign below, and give address and date. 
To my love,

\section{Parisa}

my beautiful daughter,

\section{Parmis}

and my son,

Adrin 


\section{Abstract}

A Fatigue Damage Model Developed Based on Stiffness Degradation in Human Haversian Cortical Bone, Hossein Najmi, Master of Applied Science Thesis in Mechanical Engineering, Ryerson University, Toronto, Canada, 2007.

The present study intends to develop a fatigue damage model to assess the fatigue response of human cortical bone by incorporating stiffness degradation of bone materials as the number of loading cycles progresses. The proposed fatigue damage model is defined based on mechanical properties and biological parameters of human cortical bone subjected to repeated loads. Stiffness loss in bone and bone constituents was used as a damage index to model the response of fatigue damage. The proposed damage model in this thesis considered bone as a natural composite material consisting of Haversian osteons (fibres) embedded in interstitial bone (matrix) and separated by weak cement-line interfaces.

Predicted fatigue damage results were found in good agreement with many experimentally obtained damage results of human cortical bone. The proposed damage equation also showed a higher degree of success in damage assessment of cortical bone samples tested by different laboratories as compared to other earlier developed damage models.

The proposed damage model, for the first time, successfully correlated the mechanical and histological properties of human cortical bone with damage accumulation of bone constituents. These parameters represent mechanical and histological properties of cortical bone specimens such as osteon volume fraction, donor age, cyclic stress magnitude, secant modulus of osteons, cement line interfacial strength and other bone constituent mechanical properties.

A computer program was also developed to assess fatigue damage of cortical bone by the proposed damage model and evaluate the proposed model with experimental data extracted from the literature. 


\section{Acknowledgements}

I would like to extend my greatest thanks to my advisor Professor A. Varvani-Farahani for allowing me the opportunity to work in this field. He has provided me with the guidance, motivation, inspiration, and constant support I have needed to complete this thesis.

I would like to express my sincerest appreciation to my wife, Parisa, for her love, patience, inspiration, and unconditional support. The completion of this work would not have been possible without her. Also, my deep appreciation is given to my family and relatives who were very much a part in my completion of this work.

I would like to take this opportunity to express my deep appreciation to all the authors whose insightful and inspiring research works helped me accomplish this study. Without these invaluable resources, I would have been unable to fulfill the challenging requirements of this line of research. I would specifically like to thank Dr. Cari Whyne, director of Orthopaedic Biomechanics Laboratory at Sunnybrook Health Science Centre, for her invaluable comments on this investigation.

Finally, the financial support of NSERC of Canada, through Professor A. VarvaniFarahani of the Department of Mechanical and Industrial Engineering, Ryerson University, is greatly appreciated. 


\section{Table of Contents}

Author's Declaration .................................................................................................. ii

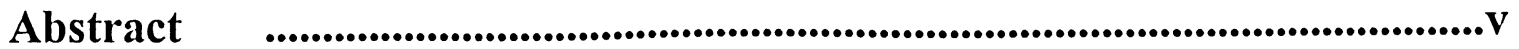

Acknowledgements ...................................................................................................... vi

Table of Contents ................................................................................................ vii

List of Figures.............................................................................................................. $x i$

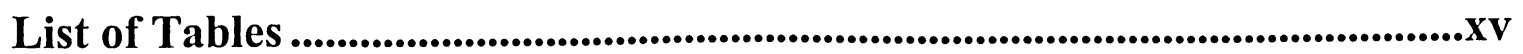

Nomenclature .............................................................................................................. Xvii

Objective and Scope............................................................................................... xviii

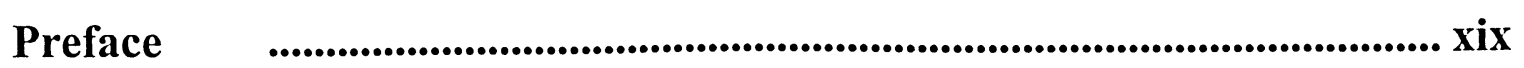

Chapter 1 Cortical Bone Structure .........................................................................1

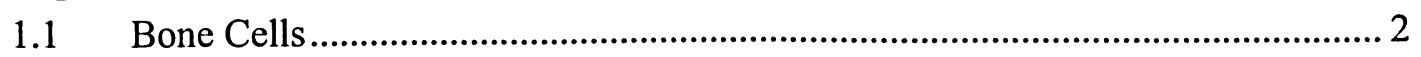

1.2 Cortical versus Trabecular Bone Structure ……….............................................. 3

$1.3 \quad$ Haversian System Cortical Bone ..................................................................... 4

$1.4 \quad$ Hierarchical Structure of Cortical Bone …………........................................ 5

1.4.1 First Level Cortical Bone Structure ............................................................... 6

1.4.1.1 Woven-fibred cortical bone........................................................................6

1.4.1.2 Plexiform Cortical Bone Tissue ................................................................6

1.4.1.3 Primary Osteonal Cortical Bone Tissue ...................................................

1.4.1.4 Secondary Osteonal Cortical Bone Tissue ....................................................7

$1.5 \quad$ Second Level Cortical Bone Structure............................................................... 8

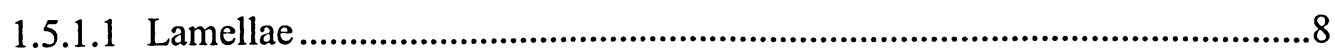

1.5.1.2 Lacunae and Canaliculi ............................................................................9

1.5.1.3 Cement Line .................................................................................................9

1.6 Third Level Cortical Bone Structure - Collagen Fibres ..................................... 9

1.6.1.1 Lamellar Type-I Collagen Orientation ..........................................................9

1.6.1.2 Mineral Packing within Collagen Fibrils ....................................................11

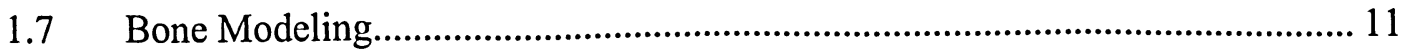


1.8 Bone Remodelling

\section{Chapter 2 Constitutive Relations in Human Cortical Bone under}

\section{Monotonic Loading.}

$2.1 \quad$ Elastic Properties ....................................................................................... 13

$2.2 \quad$ Strength of Cortical Bone ............................................................................... 17

2.3 Parameters Affecting Mechanical Properties of Bone...................................... 18

2.3.1 Strain Rate Effect (Viscoelasticity) ........................................................... 18

2.3.2 Modes of Loading ................................................................................... 19

2.3.3 Microstructure .................................................................................... 20

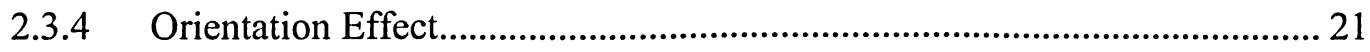

2.3.5 Effect of Mineral Composition ................................................................. 22

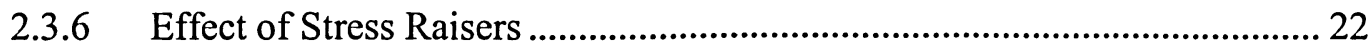

$2.4 \quad$ Bone as a Natural Composite........................................................................... 23

Chapter 3 Fatigue and Microdamage in Cortical Bone .............................25

3.1 Microdamage Mechanisms in Cortical Bone..................................................25

3.1.1 Microcracking Phenomenon ..................................................................... 26

3.1.2 The Mechanical Effects of Microcracking ................................................ 26

3.1.3 Microcracking of Bone as a Composite Material ........................................ 27

3.1.3.1 Osteon Pullout ...........................................................................................29

3.1.4 Remodelling and Repairing Process of Microcracks.................................. 29

3.2 Parameters Affecting Fatigue Damage of Human Cortical Bone ...................... 30

3.2.1 Stress Amplitude................................................................................... 30

3.2.2 Density of Micro-constituents....................................................................... 32

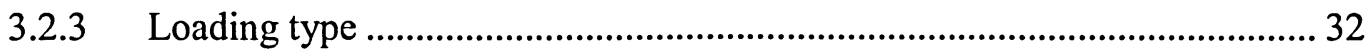

3.2.4 Microstructure and Remodelling .................................................................. 34

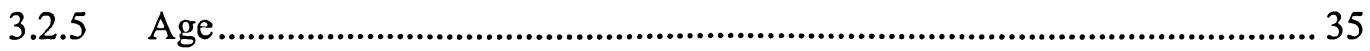

\section{Chapter 4 Analysis and Modeling of Fatigue Damage in Human Cortical} Bone

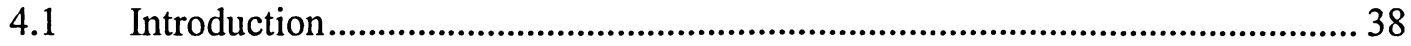

4.1.1 Fatigue in Composite Materials ................................................................... 39

4.1.2 Fatigue of Cortical Bone as a Composite Material ....................................... 40 
4.2 The Proposed Damage Model........................................................................ 42

4.2.1 Fatigue Damage Components ................................................................. 43

4.2.2 Damage Functions ................................................................................. 44

4.2.3 The Proposed Fatigue Damage Model............................................................ 47

4.2.4 Elements Affecting the Damage Assessment of Cortical Bone.................... 49

4.2.4.1 Volume Fraction of Osteons ..................................................................49

4.2.4.2 Secant Modulus of Osteons....................................................................52

4.2.4.3 Applied Cyclic Stress Versus Fatigue Life ..............................................53

4.2.4.4 Cement Line Interfacial Strength ...............................................................54

Chapter 5 Evaluation of the Proposed Fatigue Damage Model.................55

$5.1 \quad$ Evaluation Procedure ................................................................................... 55

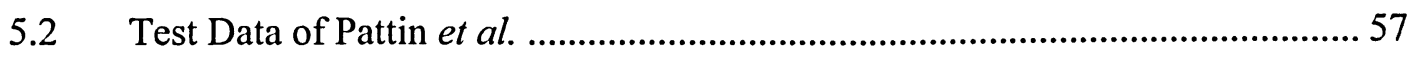

$5.3 \quad$ Test Data by Griffin et al ................................................................................ 60

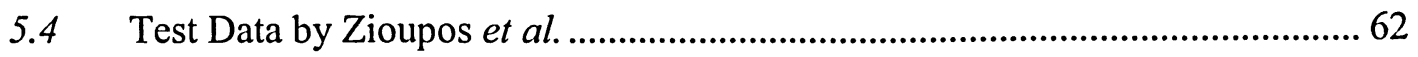

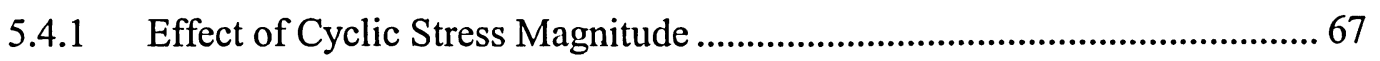

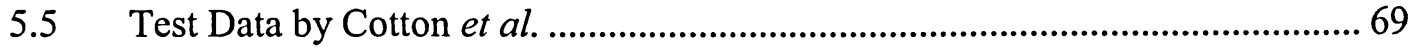

Chapter 6 Discussion of Results...............................................................71

6.1 Evaluation Results and Diagrams ................................................................. 72

6.2 Comparison of Fatigue Damage Models ............................................................. 73

6.2.1 Damage Model by Pattin et al. ..................................................................... 74

6.2.2 Damage Model by Zioupos et al.................................................................... 78

6.2.3 Damage Model by Griffin et al..................................................................... 80

Chapter 7 Conclusion and Recommendations .................................................884

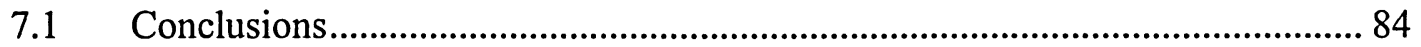

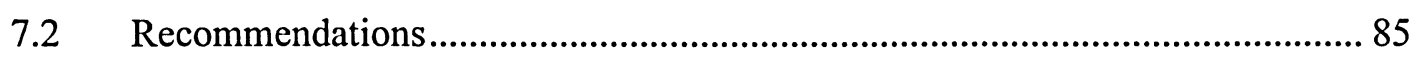

Appendix A Computer Code for Model Evaluation .....................................887

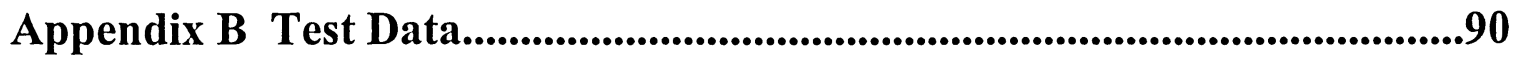

Data of Zioupos et al [25] ........................................................................................ 90

Data of Zioupos and Casinos [25] continued.............................................................. 91

Data of Zioupos and Casinos [25] continued............................................................. 92 
Data of Zioupos and Casinos [25] continued.......................................................... 93

Data of Zioupos and Casinos [25] Continued...................................................... 94

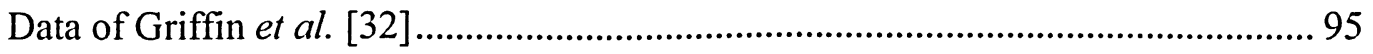

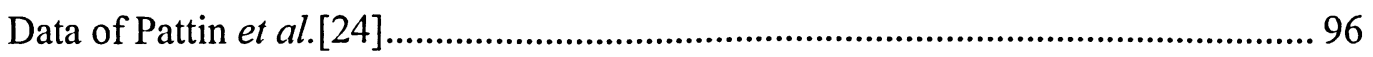

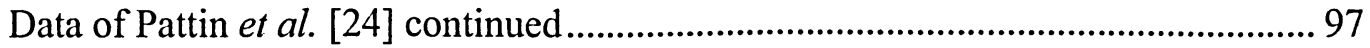

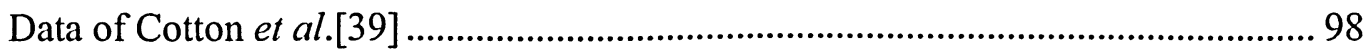

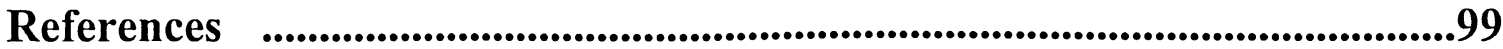




\section{List of Figures}

Fig. 1.1: Illustration of a sample human cortical bone (human femur) [Gray's Anatomy].

1

Fig. 1.2: The sequence of activation, resorption, reversal and formation as stages of the remodelling process in human cortical bone.

Fig. 1.3: Cortical and cancellous (trabecular) bone in human femur. 3

Fig. 1.4: Heversian system cortical bone, bone cells and connecting canals (lacunae).

Fig. 1.5: Hierarchical structure in human cortical bone [4]. 6

Fig. 1.6: Secondary Osteon (Haversian system) Compact bone. Notice the Haversian canals in (b) (large dark circles) and the rings of lamellae that surround them to form an osteon. The smaller dark circles are lacunar spaces within the bone [1]. 8

Fig. 1.7: Classification of osteons: (a) Bright field (Type T) osteon, (b) alternating (Type A) osteon, (c) dark field (Type L) osteon [5].

Fig. 1.8: A model of packing of the collagen fibres mineral crystals (plates) by Weiner and Traub [9].

Fig. 2.1: Preferred directions for measuring mechanical properties of cortical bone. 14

Fig. 2.2: Human femur and human tibia bones 16

Fig. 2.3: A typical stress-strain curve of cortical bone presenting the large variation of resistance to tensile loading (Reilly and Burstein [13]).

Fig. 2.4: (Top left) Stress-strain curve of bone loaded in a single pull to fracture. (Top right) A bone specimen loaded and unloaded three times and then loaded to failure. (Bottom) Mild steel loaded beyond the yield point $\mathrm{Y}$ and then unloaded twice, at A and B (Currey [12]).

Fig. 2.5: Load-deformation curve for cortical bone under compression [2].

Fig. 2.6: Osteon (Haversian system) quantity and density and its ratio to interstitial lamellae and primary bone have a great effect on the mechanical properties of cortical bone.

Fig. 2.7: Haversian osteons consist of layered lamellae with different collagen orientations.

Fig. 2.8: Some of the stress raisers in cortical bone (circled). .23 
Fig. 2.9: Hierarchical levels of structure in a human femur [11].

Fig. 3.1: Schematic representation of microcrack arrested between the cement lines.

Fig. 3.2: A stress-strain hysteresis loops of human cortical bone tested under stress-controlled condition (Pattin et al. [24]).

Fig. 3.3: Crack growth in different materials (image from Currey [12]) ......................................... 28

Fig. 3.4: a) Schematic diagram illustrating the mechanism of osteon pullout [26]. Tensile loads produce a crack that advances transversely across intact osteons embedded in the interstitial matrix. The matrix shears from the osteon cement line, resulting in debonding and osteonal crack bridging. Eventually the bridging osteons fail in tension b) image of an osteon pulled out from bone tissue.

Fig. 3.5: Dimensions of bone specimens tested by Swanson et al. [28] 31

Fig. 3.6: The median s-n curve for all cortical bone samples tested by Swanson et al. [28]. 31

Fig. 3.7: Average stiffness loss profiles for specimens subjected to Zero-Tension (0T), ZeroCompression (0C), and Zero-Torsion (0R) [30].

Fig. 3.8: Fracture surfaces of cortical bone specimens subjected to cyclic loading along with the schematic representation of micro-crack development in specimens subjected to ZeroTension (Mode I) (a), Zero-Compression (Mode II) (b), and Zero-Torsion (Modes II \& III) (c) loading [30].

Fig. 3.9: Age as a function of life cycles to failure. Fatigue life exponentially decreases with age [31].

Fig. 3.10: Modulus degradation curves for young and old donors. Young donors display a continuous drop in stiffness, while old donors show three stages of fatigue damage [31] ... 37

Fig. 4.1: Secondary osteons (Haversian system) type of cortical bone is shown. Osteons are considered to be acting like fibres embedded in interstitial bone as matrix in bone as a natural composite.

Fig. 4.2: A tri-stage fatigue behaviour seen in most composite materials as well as tensile fatigue of cortical bone (data from Pattin et al. [24]).

Fig. 4.3: Cortical bone as a composite material. Complete osteons are considered to act as fibres and interstitial bone and osteon fragments are taken as matrix. Cement lines separate the matrix from the osteon fibres. 
Fig. 4.4: A typical fatigue damage response in the matrix component of cortical bone. Damage (stiffness drop) grows rapidly at the beginning due to sudden microdamage diffusion. Then it merges to a gentle linear growth typical of most cortical bone test results

Fig. 4.5: Damage behaviour in fibres (osteons) component of cortical bone. Damage gently accumulates in a linear mode. Then there is a sudden drop in stiffness leading to the failure of the specimen 46

Fig. 4.6: A typical damage versus life fraction diagram of cortical bone under tensile fatigue loading presents (a) matrix and (b) fibres and (c) the overall fatigue damage. 48

Fig. 4.7: Relationship between the percent primary bone and age. Data from Taylor and Lee [35] (shown with circles). a) Function $\exp (-0.04$. Age) shows good agreement to test results. b) diagram of $V_{f}$ (osteon volume fraction) in terms of 'Age' equation (4.16). .51

Fig. 4.8: Osteon fragments are old osteons partially replaced by new remodelled bone. Osteon fragments (as part of the interstitial bone) are considered the matrix in cortical bone. 52

Fig. 5.1: Process chart for the evaluation of the proposed fatigue damage model against test data....56

Fig. 5.2: Pattin et al. [24] test data compared with predicted damage based on the proposed fatigue damage model, Eq. (4.13). 58

Fig. 5.3: Pattin et al. [24] test data compared with predicted damage based on the proposed fatigue damage model, Eq. (4.13). 59

Fig. 5.4: Griffin et al. [32] test data compared with predicted damage based on the proposed fatigue damage model, Eq. (4.13).

Fig. 5.5: Zioupos and Casinos [25] test data compared with predicted damage based on the proposed fatigue damage model, Eq. (4.13).

Fig. 5.6: Zioupos and Casinos [25] test data compared with predicted damage based on the proposed fatigue damage model, Eq. (4.13).

Fig. 5.7: Zioupos and Casinos [25] test data compared with predicted damage based on the proposed fatigue damage model, Eq. (4.13).

Fig. 5.8: Zioupos and Casinos [25] test data compared with predicted damage based on the proposed fatigue damage model, Eq. (4.13). 66

Fig. 5.9: Effect of stress magnitude on fatigue damage- $n / \mathrm{N}_{\mathrm{f}}$ diagrams for test specimens of a) 27year-old and b) 56-year-old donor ages. 68 
Fig. 5.10: Variation of slope $m$ with cyclic stress magnitudes for cortical bone test specimens from donor age of a) 27 years, b) 56 years, c) presents slope $m$ vs. age diagram for above ages.

Fig. 5.11: Cotton et al. test data [39] compared with predicted damage based on the proposed fatigue damage model, Eq. (4.13).

Fig. 6.1: Proposed damage model is compared to fatigue model of Pattin et al. using their test data in [24]

Fig. 6.2: Proposed damage model is compared to fatigue model of Pattin et al. in [24] using test data from Cotton et al. [39].

Fig. 6.3: Proposed damage model is compared to fatigue model of Pattin et al. in [24] using test data from Griffin et al. [32].

Fig. 6.4: Proposed damage model is compared to fatigue model of Zioupos and Casinos using their test data in [25].

Fig. 6.5: Proposed damage model is compared to fatigue model of Zioupos and Casinos in [25] using test data from Pattin et al. [24].

Fig. 6.6: Proposed damage model is compared to fatigue model of Griffin et al. using their test data in [32] 


\section{List of Tables}

Table 1.1: Cortical bone structural organization along with approximate physical scales.

Table 2.1: Elastic stiffness coefficients for human femur and human tibia cortical bones [27]......... 16

Table 2.2: Elastic moduli (in GPa) for human Haversian cortical bone [2] ..................................... 17

Table 2.3: Strength properties of (strengths in MPa) human Haversian cortical bone [2]................. 17

Table 4.1: Shear Strengths of cortical bone cement lines [1] ......................................................5

Table 5.1: Test data and parameters of Pattin et al......................................................................5

Table 5.2: Test data and parameters from Griffin et al. [14] ............................................................6 60

Table 5.3: Test data and parameters from Zioupos and Casinos [25] ..............................................6 62

Table 5.4: Test data and parameters from Cotton et al. [11].......................................................... 70

Table 6.1: Constants of the equation (6.8) by Zioupos and Casinos [25]........................................... 78 


\section{Nomenclature}

A Donor age

$C_{i j} \quad$ Stiffness coefficients

D Damage $\left(1-E / E_{0}\right)$

$D_{f} \quad$ Fibre (osteon) damage function

$D_{m} \quad$ Matrix (interstitial bone) damage function

$E^{*} \quad$ Initial (prior to fatigue testing) tangent modulus of elasticity

$E_{f}^{*} \quad$ Ratio of osteon volume fraction and osteon modulus of elasticity to initial modulus of elasticity of bone $\left(E_{f} V_{f} / E_{0}\right)$

$E_{0} \quad$ Initial (maximum) secant modulus of elasticity

$E_{c} \quad$ Composite modulus of elasticity

$E_{f} \quad$ Fibre (osteon) modulus of elasticity

$E_{m} \quad$ Matrix (interstitial bone) modulus of elasticity

$f \quad$ Interfacial (cement line) strength parameter

$G \quad$ Shear modulus

$m \quad$ Variation of stage II slope of D-n curve for a given donor age

$n \quad$ Number of cycles

$N_{f} \quad$ Fatigue life of the cortical bone specimen

$r \quad$ Normalized applied cyclic stress

$S_{i j} \quad$ Compliance coefficients

$V_{f} \quad$ Fibre (osteon) volume fraction

$V_{m} \quad$ Matrix (interstitial bone) volume fraction

a Osteon Volume fraction correction coefficient 
$\Delta \sigma \quad$ Applied cyclic stress range

$\sigma_{i} \quad$ Second-rank stress tensor

$\alpha_{n} \quad$ Fraction of the apatite crystallites

$\varepsilon_{i} \quad$ Second-rank infinitesimal strain tensor

$v \quad$ Poison's ratio

$\tau_{d b} \quad$ Maximum debonding shear strength of cement lines

$\tau_{i} \quad$ Maximum interfacial shear strength of cement lines

$\varphi_{n} \quad$ Angle of collagen minerals from the direction of stressing

\section{Definitions}

Canaliculi: Small channels found in ossified bone and used for exchange of nutrients and waste by osteocytes (single canaliculus).

Cartilage: A firm, flexible connective tissue which is the main component of the articulating surfaces of joints and of structures such as the larynx and respiratory tract and the external ear.

Lacunae: $\quad$ Small spaces containing osteocytes in bone (single lacuna).

Osseous: $\quad$ Of or related to bone; composed of bone; resembling bone

Osteoblasts: Mononucleate cells that are responsible for bone formation and produce osteoid. They are also responsible for mineralization of the osteoid matrix.

Osteoclasts: A type of bone cell that removes bone tissue by removing the mineralized matrix.

Osteocytes: Star-shaped cells which are the most abundant cell found in bone. They are used for exchange of nutrients and waste

Osteoid: $\quad$ A protein mixture which is secreted by osteoblasts. When it mineralizes, it becomes bone. Osteoid is primarily composed of Type-I collagen.

Periosteum: Membrane of tough fibrous tissue covering the bones. 


\section{Objective and Scope}

In order to determine the causes of repetitive stress injuries, the mechanism for fatigue damage accumulation in cortical bone is of great interest to researchers and clinicians. Investigations on the fatigue of structural materials and engineering composites are well established. However, the fatigue processes of bone are not well understood. Cyclic loading of cortical bone produces noticeable damage in the form of microcracks which are thought to degrade the mechanical integrity of bone structure.

The present study intends to develop a damage model to assess fatigue response of human cortical bone by incorporating stiffness degradation of bone materials as the number of loading cycles progresses. The damage model is defined based on mechanical properties and biological parameters of human cortical bone subjected to repeated tensile loads. Stiffness loss in bone and bone constituents is used as a damage index to model the response of fatigue damage. To analyze and model the fatigue damage response, bone is considered as a natural composite material consisting of Haversian osteons (fibres) embedded in interstitial bone (matrix) and separated by weak cement lines interfaces.

The proposed damage model consists of parameters which are readily available and very inexpensive to obtain. These parameters represent mechanical and histological properties of cortical bone specimens including osteon volume fraction, donor age, cyclic stress magnitude, secant modulus of osteons, cement line interfacial strength and mechanical properties of bone constituents. 


\section{Preface}

The following provides a brief description of materials covered in the chapters of this thesis.

Chapter 1 presents an introduction to cortical bone hierarchical structure and the important biological processes in cortical bone such as bone remodelling, which have been found to be influential in changing the mechanical properties of cortical bone.

Chapter 2 discusses the constitutive relations in human cortical bone under monotonic loads. The mechanical and biological parameters affecting the mechanical properties of cortical bone are also reviewed. Typical values of major strength properties and bone moduli are presented as well.

In Chapter 3, microdamage and microcracking in cortical bone are discussed. Fatigue damage accumulation and various parameters affecting the fatigue response of cortical bone are also presented. The analogy of bone to composite materials and its effects on the fatigue response of cortical bone are further discussed. In this chapter, a brief overview of the various parameters affecting damage accumulation and fatigue life of cortical bones are also presented. Related test results and theories are also reviewed.

Chapter 4 presents the analysis of fatigue damage of human cortical bone and proposes a mathematical model for predicting the damage behaviour. The fatigue damage analysis presented in this chapter aims to help researchers and clinicians to assess damage progress in micro-constituents of bone structure at various stress magnitudes and different human age groups as the number of loading cycles increases. In this chapter, fatigue damage behaviour in composite materials has been used as a fundamental concept to develop the proposed damage model for cortical bone.

In Chapter 5, the proposed fatigue damage model for human cortical bone is assessed and compared against real test results. The test data (which are from various age groups) are obtained from literature and are for different magnitudes of cyclic stresses. Test data presented in this chapter have been performed by four different laboratories and are described in details. 
Chapter 6 discusses the results of the assessment of the proposed damage model with real test data. Assessment diagrams from Chapter 5 are discussed in detail. In this chapter, the proposed damage model is also compared with some widely known previously developed fatigue damage models of cortical bone by other researchers. The advantages of the proposed damage model over these models are highlighted.

Finally conclusion and recommendations for future investigations on the fatigue damage assessment of human cortical bone are presented in Chapter 7.

Appendix A presents the details of the computer program developed to assess fatigue damage of cortical bone and Appendix B lists fatigue damage data of cortical bone conducted by different laboratories. 


\section{Chapter 1}

\section{Cortical Bone Structure}

Human bone has the ability to withstand thousands of pounds per square inch, to absorb extreme measures of shock, and to act as impenetrable armour. Bones function to move, support, and protect the body, produce red and white blood cells and store minerals. Bones come in a variety of shapes and have a complex internal and external structure, allowing them to be lightweight yet strong and hard, while fulfilling their many other functions. One of the tissues that make up bones is the mineralized osseous tissue, also called bone tissue, that gives bone rigidity and honeycomb-like three-dimensional internal structure. Other tissue types found in bones include marrow, the periosteum, nerves, and blood vessels. Fig. 1.1 illustrates a human femur (cortical bone) taken from Gary's Anatomy.

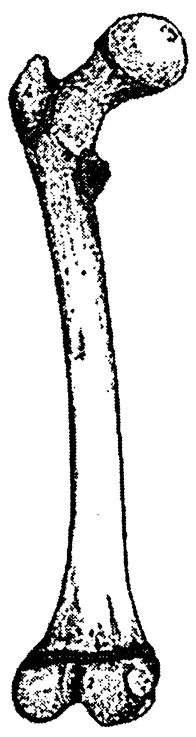

Fig. 1.1: Illustration of a sample human cortical bone (human femur) [Gray's Anatomy].

Bone is a stiff material and resembles a natural composite consisting of organic (collagen protein) and inorganic (mineral) materials. The inorganic component consists of Hydroxyapetite (HA) which is plate-like, 20 to $80 \mathrm{~nm}$ long and 2 to $5 \mathrm{~nm}$ thick. This component gives bone its solid and hard consistency and constitutes 60 to 70 percent of the

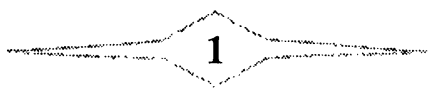


dry weight of bone. Bone also contains water, which is very influential on its mechanical properties [1].

\subsection{Bone Cells}

The cells which form the bone structure are osteoblasts and osteocytes. Osteoblasts derive from bone-lining cells and are responsible for the formation of bone. They initially form the collagenous matrix, osteoid, in which minerals are later deposited. Osteocytes, star-shaped cells, are the most abundant cells found in bone. Once osteoblasts are trapped in the matrix they form, they become osteocytes. Osteocytes are networked to each other and occupy tiny canals of canaliculi, which are used to exchange nutrients and waste. The space that an osteocyte occupies is called a lacuna. Bone lining cells cover all surfaces of bones, including the blood channels, forming a thin continuous sheet that controls the movement of ions between the body and the bone. The layer of the cells on the outside of the bone is called periosteum while the layer of the cells on the inside of the bone is called endosteum [2].

Osteoclasts are bone cells responsible for takin away bone tissue by removing the bone's mineralized matrix. This process is known as bone resorption. Osteoclasts and osteoblasts are instrumental in controlling the amount of bone tissue. Osteoblasts form the bone structure while osteoclasts resorb the bone material. Fig. 1.2 presents the stages of remodelling process in human cortical bone which are: activation, resorption, reversal and formation.

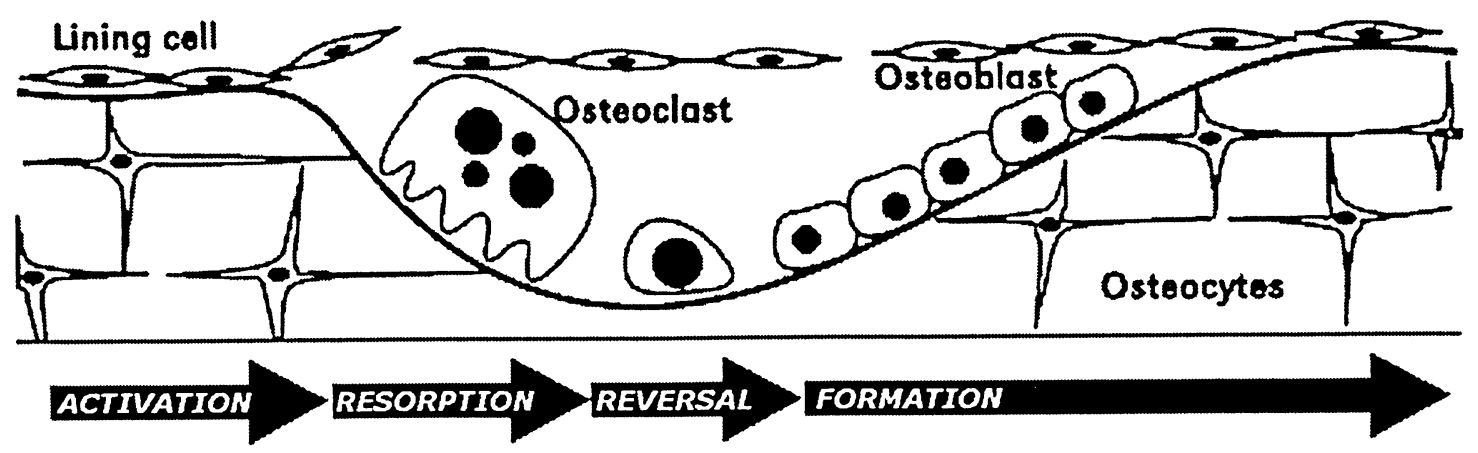

Fig. 1.2: The sequence of activation, resorption, reversal and formation as stages of the remodelling process in human cortical bone [2]. 


\subsection{Cortical versus Trabecular Bone Structure}

Bone in human and other mammal bodies is generally classified into two types 1) Cortical bone, also known as compact bone, and 2) Trabecular bone, also known as cancellous or spongy bone. These two types are classified based on porosity and the unit microstructure. Cortical bone is much denser with a porosity ranging between $5 \%$ and $10 \%$. The word "cortex" or cortical usually refers to the outer layer of a structure. Therefore cortical bone is the outer layer of the skeleton and is found primary in the shaft of long bones and forms the outer shell around cancellous bone at the end of joints and the vertebrae [1]. A cortical (compact) shell around cancellous (spongy) bone of a human femur is shown in Fig. 1.3. This outer shell of bone must withstand physiological forces applied to bone structure during daily activities. The compact bone is extremely high in mineral content and its layers are compact and very tightly connected.

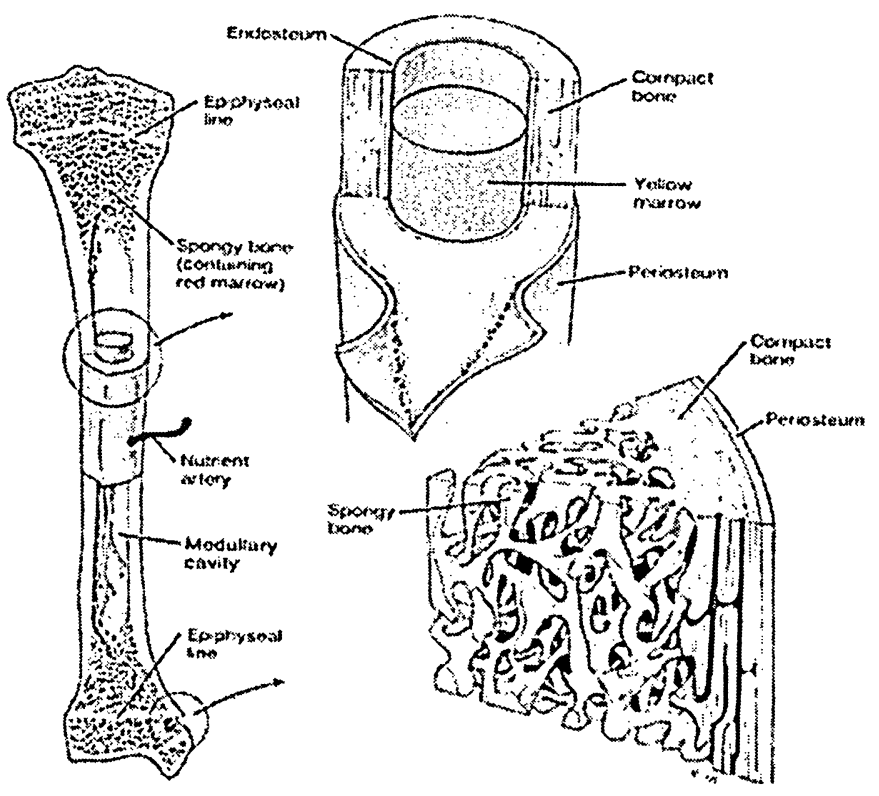

Fig. 1.3: Cortical and cancellous (trabecular) bone in human femur.

[Basic Medical Anatomy, by Alexander Spence]

On the other hand, the structure of trabecular or cancellous bone (also known as spongy bone) is porous. The spaces in the cancellous bone act as supporting struts (see Fig. 1.3). Spongy or cancellous bone is lighter than cortical bone, but cannot take as much strain. Thus, bones made of primary cortical bone possess a greater risk of fractures while strategically 
supporting the surrounding bone cortex. In another word, the structure of the bone shaft gains a high strength due to cortical bone surrounding light spongy structure of trabecular bone in the core of the bone shaft.

\subsection{Haversian System Cortical Bone}

Cortical Bone is made up of Haversian system. In simple terms, a Haversian system can be thought of as a pillar which runs parallel to the long axis of a bone acting as column in bone structure. The system, however, is made up of several layers. An outstanding analogy is to think of a tree. A tree grows vertically and its cross-section contains many "rings". Rings in bone hug each other; just as tree rings do (see Fig. 1.4). There are several reasons for this particular architecture [3]:

- Similar to other cells, osteoblasts and osteocytes need nutrients from the blood. Diffusion of nutrient materials for life can never take place through a calcified matrix. Bone is programmed to create a pattern of canals, which allow for the passage of nutrients.

- As stated earlier, the Haversian system is parallel to the long axis of a bone. It is made of circular layers, or rings of bone. These layers are called Lamellae. A hollow canal passing through these layers is referred as the central Haversian canal. Within this canal, there is a major blood vessel supplying nutrients.

- There are several small spaces (cavities) within each lamella. One can think of them as a living housing for bone cells. Each space is called a lacuna (plural is lacunae).

- From these lacunae, small canals lead to the central Haversian canal. Osteocytes have a cell body and, like spiders, they have several legs. These legs lie within the canals, and nutrients can enter them, and pass into the cell body to the bone structure. Additionally, these legs or extensions, not only communicate with the blood vessels, but with other cells. Therefore, cells can exchange nutrients with each other, and with the life-giving blood vessels. 


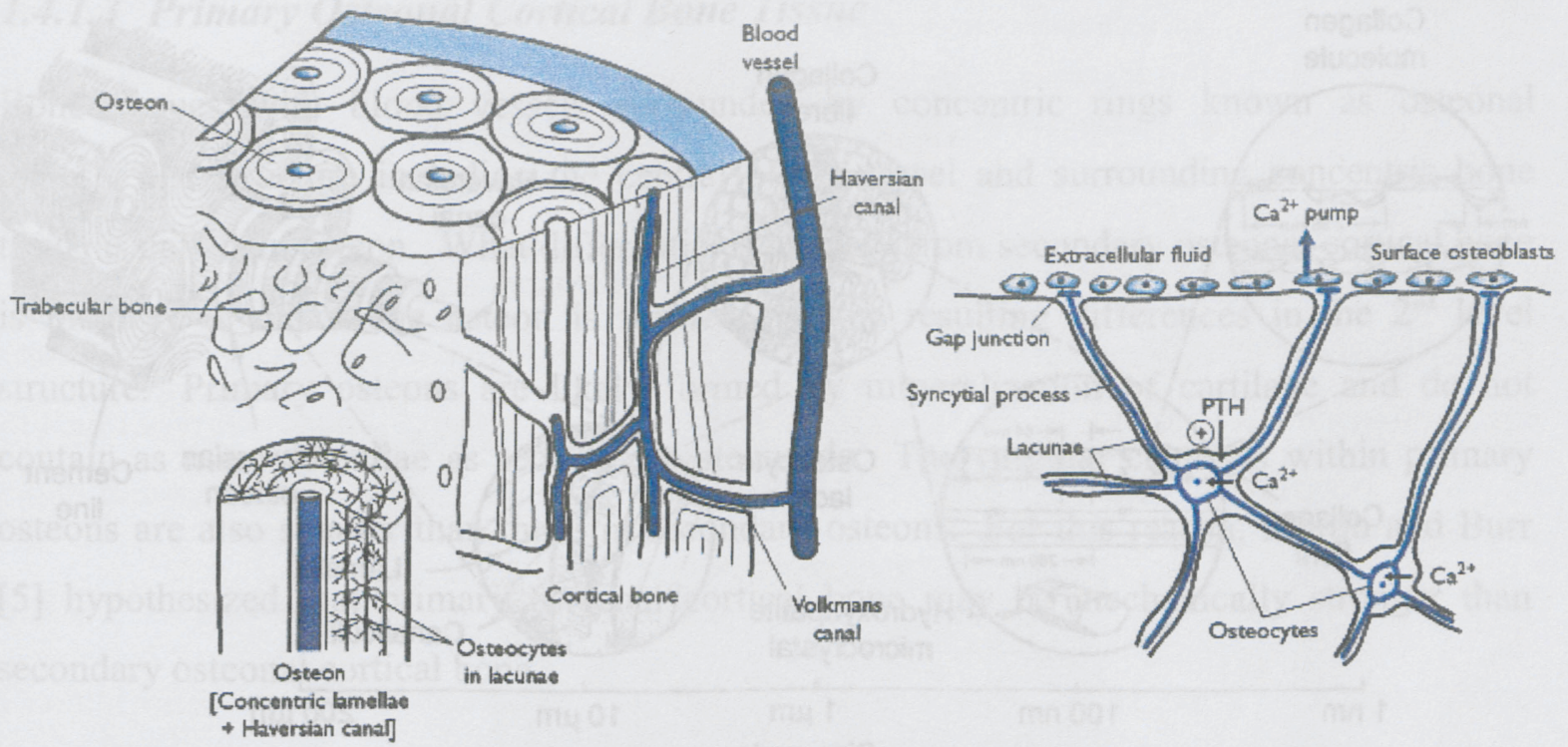

Fig. 1.4: Haversian system cortical bone, bone cells and connecting canals (lacunae). [2].

\subsection{Hierarchical Structure of Cortical Bone}

As with all biological tissues, cortical bone has a hierarchical structure. This means that cortical bone contains many different structures that exist on many levels of scale. The hierarchical organization of cortical bone is given in Table 1.1. Fig. 1.5 schematically represents the hierarchical structure of human cortical bone and the size of its microconstituents. The first and second organization levels verify that there are different types of cortical bones for different species and age groups. Note that the third level bone is composed of type-I collagen fibre-mineral composite.

Table 1.1: Cortical bone structural organization along with approximate physical scales.

\begin{tabular}{|c|l|c|}
\hline Level & \multicolumn{1}{|c|}{ Cortical Structure } & Size Range \\
\hline 0 & Solid Material & $>3000 \mu \mathrm{m}$ \\
\hline 1 & $\begin{array}{l}\text { Secondary Osteons } \\
\text { Primary Osteons } \\
\text { Plexiform } \\
\text { Interstitial Bone }\end{array}$ & 100 to $300 \mu \mathrm{m}$ \\
\hline 2 & $\begin{array}{l}\text { Lamellae } \\
\text { Lacunae } \\
\text { Cement Lines }\end{array}$ & 3 to $20 \mu \mathrm{m}$ \\
\hline 3 & Collagen-Mineral Composite & 0.06 to $0.6 \mu \mathrm{m}$ \\
\hline
\end{tabular}




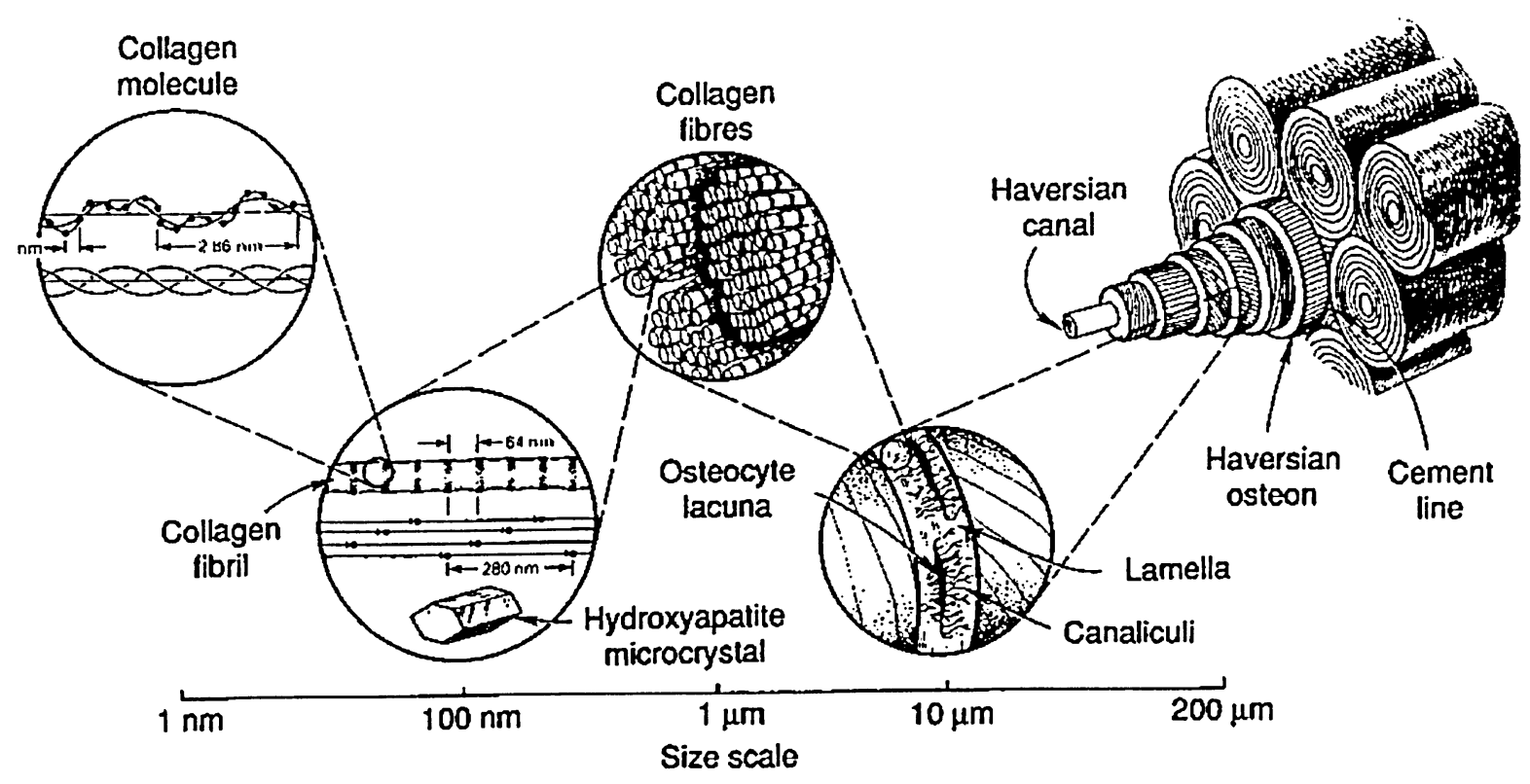

Fig. 1.5: Hierarchical structure in human cortical bone [4].

\subsubsection{First Level Cortical Bone Structure}

Martin and Burr [5] categorized four types of structures with the first structural level. These types are woven bone, primary bone, plexiform bone, and secondary bone.

\subsubsection{Woven-fibred cortical bone}

Woven bone does not contain osteons like primary and secondary bone, nor does it contain the brick-like structure of plexiform bone. Woven bone tissue is often found in very young growing skeletons under the age of 5 and is the most disorganized bone tissue. It is only found in the adult skeleton in cases of trauma or disease, most frequently occurring around bone fracture sites. Woven bone is believed to be less dense because of the loose and disorganized packing of the type-I collagen fibres [5].

\subsubsection{Plexiform Cortical Bone Tissue}

Like woven bone, plexiform bone is formed more rapidly than primary or secondary lamellar bone tissues. However, unlike woven bone, plexiform bone offers better mechanical support for longer periods of time. Because of this, plexiform bone is primarily found in large rapidly growing animals such as cows or sheep. Plexiform bone is rarely seen in human. 


\subsubsection{Primary Osteonal Cortical Bone Tissue}

Bone tissues with blood vessels surrounded by concentric rings known as osteonal bones. The structure including the central blood vessel and surrounding concentric bone tissue is called an osteon. What differentiates primary from secondary osteonal cortical bone is the way in which the osteon is formed and the resulting differences in the $2^{\text {nd }}$ level structure. Primary osteons are likely formed by mineralization of cartilage and do not contain as many lamellae as secondary osteons do. The vascular channels within primary osteons are also smaller than those in secondary osteons. For this reason, Martin and Burr [5] hypothesized that primary osteonal cortical bone may be mechanically stronger than secondary osteonal cortical bone.

\subsubsection{Secondary Osteonal Cortical Bone Tissue}

Secondary osteons are formed by replacement of existing bone. Secondary bone results from a process known as remodelling. The Haversian canal in the center of the osteon has a diameter ranging between 50 to $90 \mu \mathrm{m}$. The Haversian canal contains a blood vessel typically $15 \mu \mathrm{m}$ in diameter [1]. In secondary bones, osteons are separated from each other by cement lines. Collagen fibres and canaliculi do not cross cement lines. Osteons are connected to each other and the periosteum by oblique channels called Volkmann's canals. Fig. 1.6 shows the cortical bone structure and its secondary osteons. This level of bone structure is further discussed in the next chapters as it is the cornerstone of the proposed fatigue damage model in this thesis. 


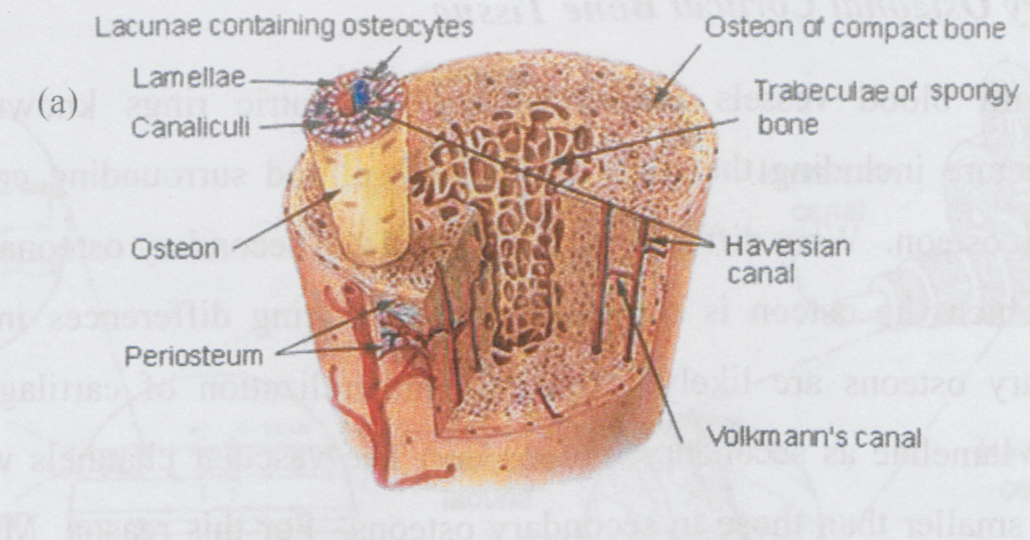

(b)

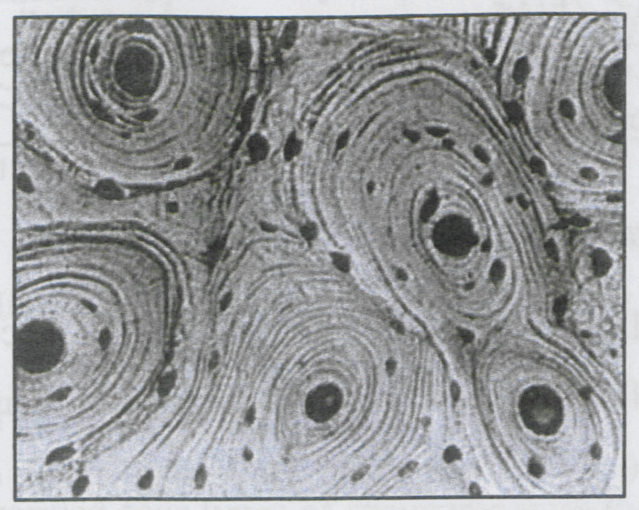

Fig. 1.6: Secondary Osteon (Haversian system) Compact bone. Notice the Haversian canals in (b) (large dark circles) and the rings of lamellae that surround them to form an osteon. The smaller dark circles are lacunar spaces within the bone [1].

\subsection{Second Level Cortical Bone Structure}

The second level cortical bone structure consists of those entities constructing the osteons in primary and secondary bones and the "bricks" in plexiform bone. Within osteonal bone (primary and secondary) the four major matrix second level structural entities are lamellae, osteocyte lacunae, osteocyte canaliculi, and cement lines.

\subsubsection{Lamellae}

Lamellae are bands or layers of bone generally between 3 and $7 \mu \mathrm{m}$ in thickness. The lamellae are arranged concentrically around the central Haversian canal in osteonal bone (see Fig. 1.6a). In plexiform bone, the lamellae are sandwiched in between non-lamellar bone layers. The lamellae in osteonal bone are separated by thin inter-lamellar layers in which the 
orientation of bone mineral may be altered. Lamellae contain type-I collagen fibres and minerals.

\subsubsection{Lacunae and Canaliculi}

The osteocyte lacunae and canaliculi are actually holes within the bone matrix containing osteocyte cells. Osteocytes evolve from osteoblasts which become entrapped in bone matrix during the mineralization process. Osteocyte lacunae have ellipsoidal shapes. Canaliculi are small tunnels which connect one lacuna to another lacuna (as shown in Fig. 1.6a).

\subsubsection{Cement Line}

Cement lines are only found in secondary bone because they are the result of a remodelling process. The cement line occurs where the bone resorption ends and bone formation begins (see Fig. 1.5). Cement lines are about 1 to 5 microns in thickness. These lines are believed to be type-I collagen deficient structures. Schaffler et al. [6] found that cement lines were less mineralized than the surrounding bone tissues. Cement lines can also arrest crack growth in bone [1].

\subsection{Third Level Cortical Bone Structure - Collagen Fibres}

Third level contains the basic type-I collagen fibres and the mineral composite. This level of bone structure has the most impact on mechanical properties such as fatigue resistance of bone.

\subsubsection{Lamellar Type-I Collagen Orientation}

Ascenzi and Bonucci [7] reported that fibres within the same lamella are predominantly parallel to one another and have a preferred orientation within the lamellae. The orientation of collagen fibres between lamellae may change up to $90^{\circ}$ in adjacent lamellae. Accordingly, three types of osteons containing three different types of lamellar sub-structures have been defined: 
a) Bright field (Type T) osteons contain lamellae with fibre bundles that are oriented parallel to the plane of the section. These bundles are oriented in a transverse spiral or circumferential hoop perpendicular to the center of the osteon.

b) Alternating (Type A) osteons contain alternating fibre bundle orientations and thus give an alternating light and dark pattern under polarized light.

c) Dark field (Type L) osteons contain lamellae with collagen fibres which are oriented perpendicular to the plane of the section, or parallel to the osteon axis. This type of osteons appears dark under polarized light.

Fig. 1.7 illustrates various osteons of Type T, Type A, and Type L with schematic of their collagen fibre orientations. Martin et al. [8] characterized a hooped osteon (type $O$ ) using circularly polarized light. They found that complex structure of osteons is mainly related to collagen fibre orientation and its mineral arrangement and that osteons affect bone mechanical properties significantly.

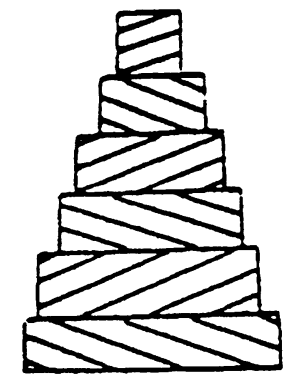

a

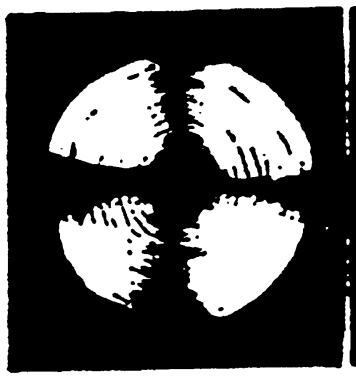

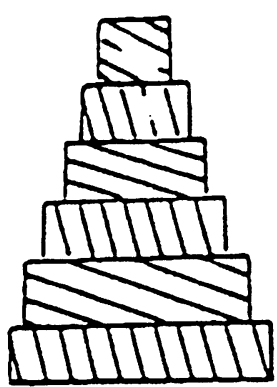

b

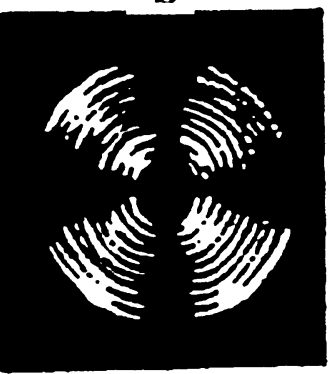

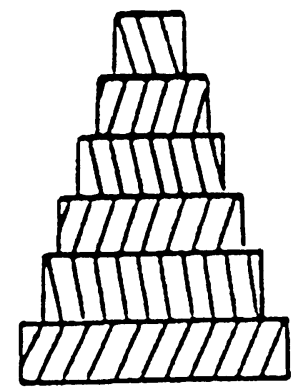

c

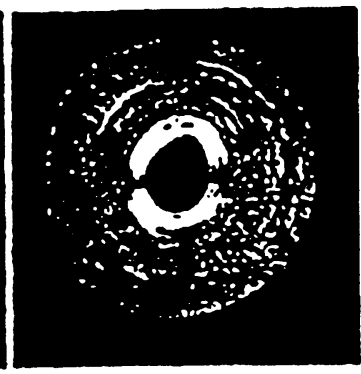

Fig. 1.7: Classification of osteons: (a) Bright field (Type $T$ ) osteon, (b) alternating (Type A) osteon, (c) dark field (Type L) osteon [5]. 


\subsubsection{Mineral Packing within Collagen Fibrils}

A very substructural review of bone from the angstrom level (mineral crystal) to the micron level (lamellae) was recently presented by Weiner and Traub [9]. They reviewed mineral structure, the mineral collagen composite, and how the mineral collagen composite fit into lamellae. Collagen fibres, with a typical width of $0.015 \mu \mathrm{m}$ and a length of $3 \mu \mathrm{m}$, packed together to form collagen fibrils. Weiner and Traub [9] observed distinct gaps within the collagen fibres where mineral crystals are formed. These gaps with collagen mineral crystals are schematically presented in Fig. 1.8. The minerals naturally form like plates and are typically 0.5 by $0.25 \mu \mathrm{m}$ in length and width respectively and have a thickness of 0.02 to $0.03 \mu \mathrm{m}$. These plates are packed into the type-I collagen fibrils. Because of the nature of the packing, the orientation of the collagen fibrils will determine the orientation of the mineral crystals.

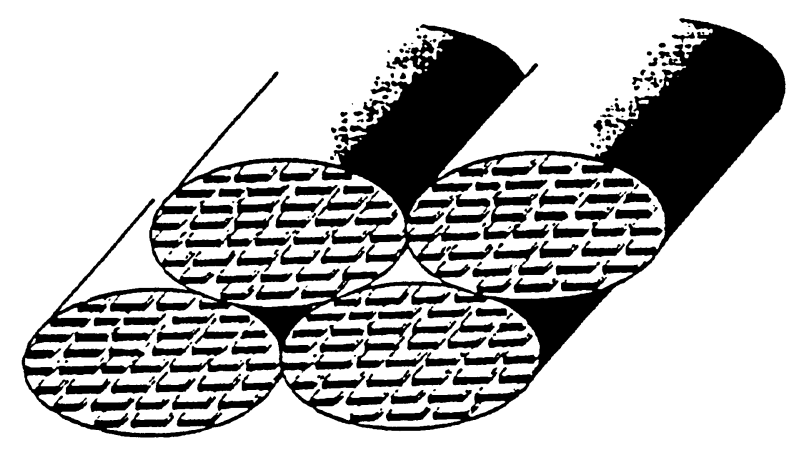

Fig. 1.8: A model of packing of the collagen fibres mineral crystals (plates) by Weiner and Traub [9].

\subsection{Bone Modeling}

In the initial bone formation and in the initial healing stage, large changes in the bone shape may be needed. This, for example, may occur because the child is growing rapidly, or because the initial hard tissue must be changed to a shape that is more functional. Modeling may cause large changes in bone structure [1]. Modelling usually results in the formation of primary osteons. 


\subsection{Bone Remodelling}

Once bone formation has completed in the first 28 years of human life, peak bone mass is maintained by a process called "remodelling". Bone adapts and remodels in response to the stress applied. Wolff's law [10] states that bones develop a structure most suited to resist the forces acting upon them, adapting both the internal architecture and the external conformation to the change under external loads. When a change in loading pattern occurs stress and strain fields in the bone change accordingly. Bone tissue seems to be able to detect the change in strain on a local base and then adapts accordingly. Haversian system is the result of bone remodelling process.

Remodelling is a process which occurs 24 hours a day, 7 days a week and involves the continuous breakdown and re-formation of bone. Bone is a live tissue just like the kidneys, heart, and other organs of the body. This live tissue continuously remodels itself to maintain maximal bone mineral density (BMD) and to repair any damage in its early stage of growth.

Remodelling consists of tearing down small parts of the bones, and then re-forming them. The breaking down portion is termed "resorption" and is performed by large cells within the bones called "osteoclasts". Osteoclasts live in the central portion of the bone. They are continually removing small (microscopic) portions of bone at the edge of the bone surface. Nearby, bone forming cells called "osteoblasts" begin to fill in the holes left behind. However, remodelling does not seem to be a perfect give and take of bone mass. The osteoblasts are less efficient at making bone than the osteoclasts are at removing it. Although the difference is slight, this is what accounts for the gradual loss of bone density (BMD) as a person ages. Any factor which causes a higher rate of bone remodelling will ultimately lead to a more rapid loss of bone mass and thus more fragile bones. 


\section{Chapter 2}

\section{Constitutive Relations in Human Cortical Bone under Monotonic Loading}

There are several factors which affect the mechanical properties of bone. For example, bone in vivo contains water which affects its elastic and strength properties than when it is dry or in vitro. In addition, the orientation of the specimen in relation to the bone and the strain rate has a great effect on the measured mechanical properties of bone specimens. It is reasonable to model cortical bone as an anisotropic, linear elastic solid with Hook's law as the appropriate constitutive equation. There are two methods of testing bone to determine the elastic properties: (1) a tensile test and constructing stress-strain curves and (2) an ultrasonic test by measuring the velocity of sound waves in bone.

\subsection{Elastic Properties}

Bone is a viscoelastic material which means its stress-strain curve is time dependent. Under quasi-static mechanical and ultrasonic tests, cortical bone responsed as an anisotropic, linear elastic solid which can be described based on Hook's law as an appropriate constitutive equation. Tensor notation for the elastic response of cortical bone is generally given as [11]:

$$
\sigma_{i}=C_{i j} \varepsilon_{j} \quad i, j=1,2,3, \ldots, 6
$$

Where $\sigma_{i}$ and $\varepsilon_{j}$ are the second-rank stress and infinitesimal strain tensors, respectively, and $C_{i j}$ is the stiffness coefficients (elastic constants). The inverse of the $C_{i j}$ (i.e. $S_{i j}$ ), is known as the compliance coefficients. Bone structure can be characterized by three main directions of 1,2 and 3 referring to radial, circumferential and longitudinal, respectively as shown in Fig. 2.1. 


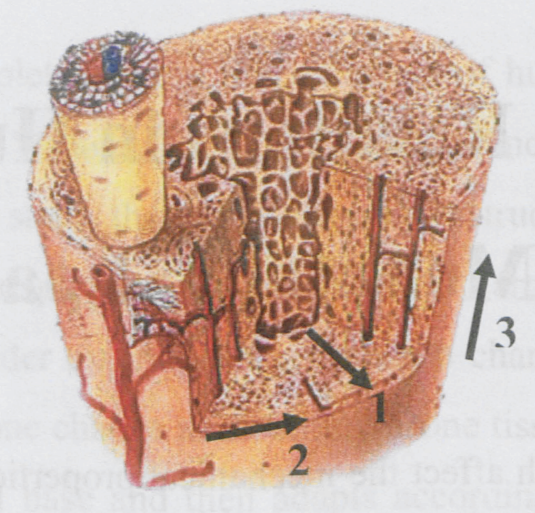

Fig. 2.1: Preferred directions for measuring mechanical properties of cortical bone.

The structure of bone is assumed to be transversely isotropic with respect to the bone longitudinal axis (the direction 3). Small difference in elastic properties between the radial axis (along the direction 1) and the transverse axis (along the direction 2) are due to the gradient in porosity from the periosteal to the endosteal sides of bone. For a transverse isotropic material, the stiffness matrix $\left[C_{i j}\right]$ is given by:

$$
\left[C_{i j}\right]=\left[\begin{array}{cccccc}
C_{11} & C_{12} & C_{13} & 0 & 0 & 0 \\
C_{12} & C_{11} & C_{13} & 0 & 0 & 0 \\
C_{13} & C_{13} & C_{33} & 0 & 0 & 0 \\
0 & 0 & 0 & C_{44} & 0 & 0 \\
0 & 0 & 0 & 0 & C_{44} & 0 \\
0 & 0 & 0 & 0 & 0 & C_{66}
\end{array}\right]
$$

where $C_{66}=1 / 2\left(C_{11}-C_{12}\right)$. Of the 12 nonzero coefficients, only 5 coefficients of $\left(C_{11}, C_{12}\right.$, $C_{13}, C_{33}$ and $\left.C_{44}\right)$ are independent due to small differences in elastic properties between the radial and transverse directions one can postulate that bone is an orthotropic material. This requires that 9 of the 12 nonzero elastic constants be independent, that is:

$$
\left[C_{i j}\right]=\left[\begin{array}{cccccc}
C_{11} & C_{12} & C_{13} & 0 & 0 & 0 \\
C_{12} & C_{22} & C_{23} & 0 & 0 & 0 \\
C_{13} & C_{23} & C_{33} & 0 & 0 & 0 \\
0 & 0 & 0 & C_{44} & 0 & 0 \\
0 & 0 & 0 & 0 & C_{55} & 0 \\
0 & 0 & 0 & 0 & 0 & C_{66}
\end{array}\right]
$$

Corresponding matrices can be written for the compliance coefficients, $S_{i j}$, based on the inverse of Eq. (2.1): 


$$
\varepsilon_{i}=S_{i j} \sigma_{j} \quad i, j=1,2,3, \ldots, 6
$$

The following relations exist between the stiffness matrix $\left[C_{i j}\right]$ components and compliance matrix $\left[S_{i j}\right]$ components:

$$
\begin{array}{ll}
C_{11}=\frac{S_{22} S_{33}-S_{23}^{2}}{S} & C_{12}=\frac{S_{13} S_{23}-S_{12} S_{33}}{S} \\
C_{22}=\frac{S_{33} S_{11}-S_{13}^{2}}{S} & C_{12}=\frac{S_{12} S_{13}-S_{23} S_{11}}{S} \\
C_{33}=\frac{S_{11} S_{22}-S_{12}^{2}}{S} & C_{12}=\frac{S_{12} S_{23}-S_{13} S_{22}}{S} \\
C_{44}=\frac{1}{S_{44}} \quad C_{55}=\frac{1}{S_{55}} & C_{66}=\frac{1}{S_{66}}
\end{array}
$$

where $S$ in above equations is given as:

$$
S=\left|\begin{array}{lll}
S_{11} & S_{12} & S_{13} \\
S_{12} & S_{22} & S_{23} \\
S_{13} & S_{23} & S_{33}
\end{array}\right|
$$

In the compliance matrix, although $S_{33}=1 / E_{3}$, where $E_{3}$ is Young's modulus in the bone axial direction, $C_{33} \neq E_{3}$, since components $C_{33}$ and $S_{33}$, are not reciprocals of one another even for an isotropic material. The relationship between the compliance matrix with technical constants measured under uniaxial or pure shear tests such as Young's modulus, $E_{i}$, shear modulus, $G_{i}$, and Poisson's ratio, $v_{i j}$, is expressed as:

$$
\left[S_{i j}\right]=\left[\begin{array}{cccccc}
\frac{1}{E_{1}} & -\frac{v_{21}}{E_{2}} & -\frac{v_{31}}{E_{3}} & 0 & 0 & 0 \\
-\frac{v_{12}}{E_{1}} & \frac{1}{E_{2}} & -\frac{v_{32}}{E_{3}} & 0 & 0 & 0 \\
-\frac{v_{13}}{E_{1}} & -\frac{v_{23}}{E_{2}} & \frac{1}{E_{3}} & 0 & 0 & 0 \\
0 & 0 & 0 & \frac{1}{G_{31}} & 0 & 0 \\
0 & 0 & 0 & 0 & \frac{1}{G_{31}} & 0 \\
0 & 0 & 0 & 0 & 0 & \frac{1}{G_{12}}
\end{array}\right]
$$

For an orthotropic material, only 9 of the above 12 nonzero terms are independent. In equation (2.7), symmetric components are: 


$$
\frac{v_{12}}{E_{1}}=\frac{v_{21}}{E_{2}} \quad \frac{v_{13}}{E_{1}}=\frac{v_{31}}{E_{3}} \quad \frac{v_{23}}{E_{2}}=\frac{v_{32}}{E_{3}}
$$

For the transverse isotropic case, Eq. (2.4) reduces to only 5 independent coefficients, since

$$
\begin{array}{lc}
E_{1}=E_{2} & v_{12}=v_{21} \quad v_{31}=v_{32}=v_{13}=v_{23} \\
G_{23}=G_{31} & G_{12}=E_{1} / 2\left(1+v_{12}\right)
\end{array}
$$

To determine the five transverse isotropic elastic constants, at least five independent measurements are required. The technical moduli must then be calculated from the full set of $C_{i j}$. Correspondingly, for orthotropic symmetry, enough independent measurements must be made to obtain all $9 C_{i j}$ coefficients. Table 2.1 presents the elastic stiffness coefficients for human tibia and human femur (shown in Fig. 2.2).

Table 2.1: Elastic stiffness coefficients for human femur and human tibia cortical bones [13].

\begin{tabular}{|l|c|c|c|c|c|c|c|c|c|}
\hline $\begin{array}{l}\text { Experiments } \\
\text { (Bone Type) }\end{array}$ & $\begin{array}{c}C_{11} \\
(\mathbf{G P a})\end{array}$ & $\begin{array}{c}\boldsymbol{C}_{22} \\
(\mathbf{G P a})\end{array}$ & $\begin{array}{c}\boldsymbol{C}_{33} \\
\mathbf{( G P a})\end{array}$ & $\begin{array}{c}\boldsymbol{C}_{44} \\
\mathbf{( G P a})\end{array}$ & $\begin{array}{c}\boldsymbol{C}_{55} \\
(\mathbf{G P a})\end{array}$ & $\begin{array}{c}\boldsymbol{C}_{66} \\
\mathbf{( G P a )}\end{array}$ & $\begin{array}{c}\boldsymbol{C}_{12} \\
(\mathbf{G P a})\end{array}$ & $\begin{array}{c}\boldsymbol{C}_{13} \\
(\mathbf{G P a})\end{array}$ & $\begin{array}{c}\boldsymbol{C}_{23} \\
(\mathbf{G P a})\end{array}$ \\
\hline Human tibia & 11.6 & 14.4 & 22.5 & 4.91 & 3.56 & 2.41 & 7.95 & 6.10 & 6.92 \\
\hline Human femur & 20.0 & 21.7 & 30.0 & 6.56 & 5.85 & 4.74 & 10.9 & 11.5 & 11.5 \\
\hline
\end{tabular}

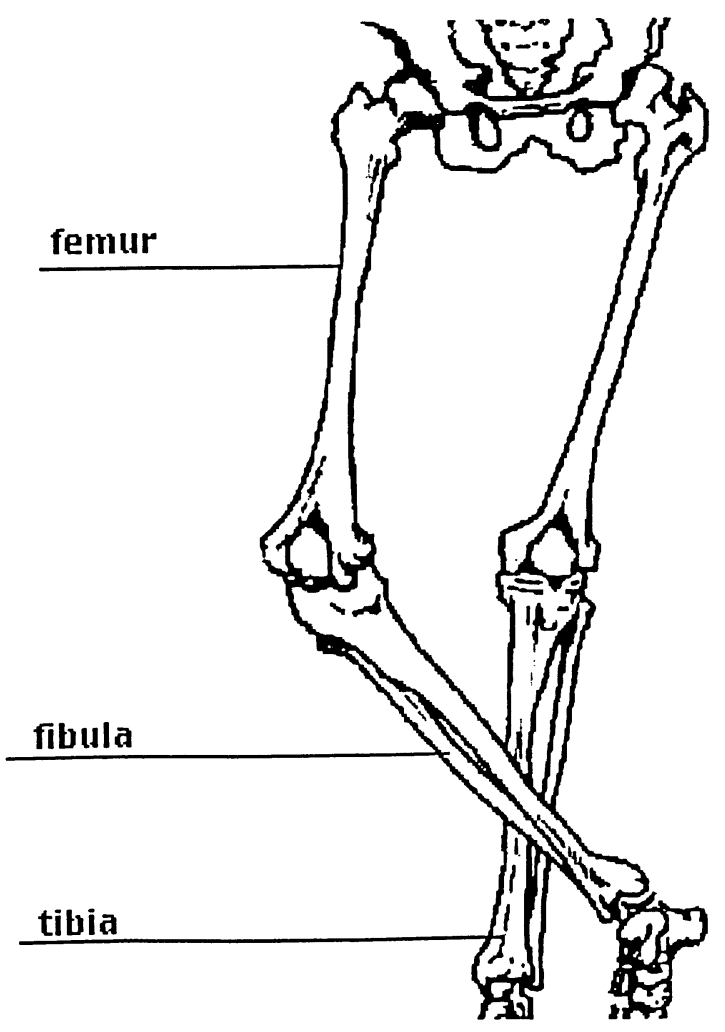

Fig. 2.2: Human femur and human tibia bones 
Table 2.2 lists the elastic mechanical properties of human Haversian cortical bone measured mechanically under tension and compression. Subscripts 1, 2, and 3 refer to the radial, circumferential and longitudinal directions relative to the long axis of the bone (See Fig. 2.1).

Table 2.2: Elastic moduli for human Haversian cortical bone [12].

\begin{tabular}{|l|c|c|c|c|c|c|c|c|c|}
\hline Property & $\begin{array}{c}E_{1} \\
(\mathbf{G P a})\end{array}$ & $\begin{array}{c}E_{2} \\
(\mathbf{G P a})\end{array}$ & $\begin{array}{c}\boldsymbol{E}_{3} \\
(\mathbf{G P a})\end{array}$ & $\boldsymbol{G}_{13}$ & $\boldsymbol{G}_{23}$ & $\boldsymbol{v}_{12}$ & $\boldsymbol{v}_{13}$ & $\boldsymbol{v}_{21}$ & $\boldsymbol{v}_{31}$ \\
\hline Tension & 12.8 & 12.8 & 17.7 & 3.3 & 3.3 & 0.53 & 0.41 & 0.53 & 0.41 \\
\hline Compression & 11.7 & 11.7 & 18.2 & 3.3 & 3.3 & 0.63 & 0.38 & 0.63 & 0.38 \\
\hline
\end{tabular}

\subsection{Strength of Cortical Bone}

Bone material is anisotropic and its properties depend on its spatial architecture and composition. Bone spatial organization is related to bone density and is characterized by the degree of bone mineralization and bone microstructural features. The mechanisms of bone failure are different from those well established for conventional isotropic materials. The average typical tensile and compressive strength values of human Haversian cortical bone are presented in Table 2.3 .

Table 2.3: Strength properties of (strengths in MPa) human Haversian cortical bone [12].

\begin{tabular}{|l|c|}
\hline Property & Human Haversian \\
\hline Tensile Strength & 158 \\
\hline Tensile yield stress & 128 \\
\hline Ultimate tensile strain & 0.042 \\
\hline Compressive strength & 213 \\
\hline Compressive yield stress & 180 \\
\hline Ultimate compressive strain & 0.26 \\
\hline Shear strength & 71 \\
\hline Shear yield stress & 53 \\
\hline
\end{tabular}

As mentioned earlier, bone mechanical properties vary in different directions (bone anisotropy). As shown in Fig. 2.3 the stress-strain response is very different in major directions for a typical long human femur cortical bone. 


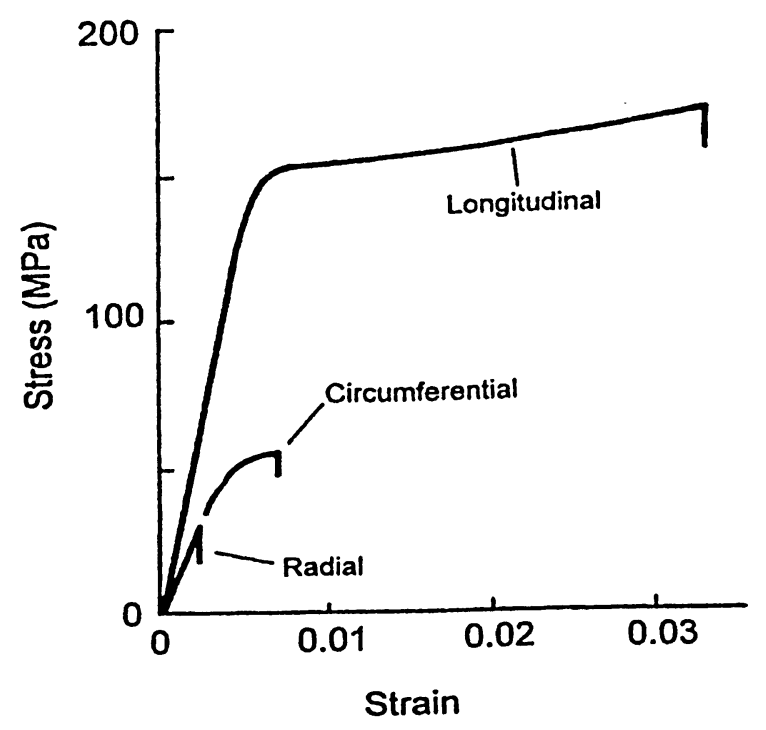

Fig. 2.3: A typical stress-strain curve of cortical bone presenting the large variation of resistance to tensile loading (Reilly and Burstein [13]).

\subsection{Parameters Affecting Mechanical Properties of Bone}

In this section, the effects of various parameters on changing the mechanical properties of bone are discussed. Bone acts like a viscoelastic natural composite. The difference in volume fractions of organic and inorganic constituents of bone and constant changing of them in the course of human life has a great effect on the mechanical properties of bone. Remodelling in bone is a well known phenomenon that through destruction and reconstruction processes constantly change the microstructure and the mechanical properties of bone. Some of the main parameters affecting the mechanical properties of cortical bone are described in the following sections.

\subsubsection{Strain Rate Effect (Viscoelasticity)}

Bone is slightly viscoelastic meaning its Young's modulus is to some extent strain-rate dependent. Carter and Caler [14] suggested that Young's modulus is affected by strain rate non-linearly (i.e. a thousand-fold increase in strain rate will result in a $40 \%$ apparent increase of Young's modulus). Hence, mechanical tests should be conducted as close to real-life strain rates as possible. 
The effect of strain rate is more prominent in the plastic region. Bone does not undergo plastic deformation like metals. The bone behaves elastically in the first region but if it is unloaded and reloaded after the stress-strain curve is flattened out, the curves are no longer super-imposable. As shown in Fig. 2.4, the slopes of the loading curves are different; the specimen is becoming more compliant each time it is loaded into the yield region. It is also clear that most of the strain is not fully recovered under the limited time. This presents the viscoelastic (time dependant) nature of bone under cyclic loading. The stress achieved does not increase much with strain, the envelope of the curves being rather flat-topped.
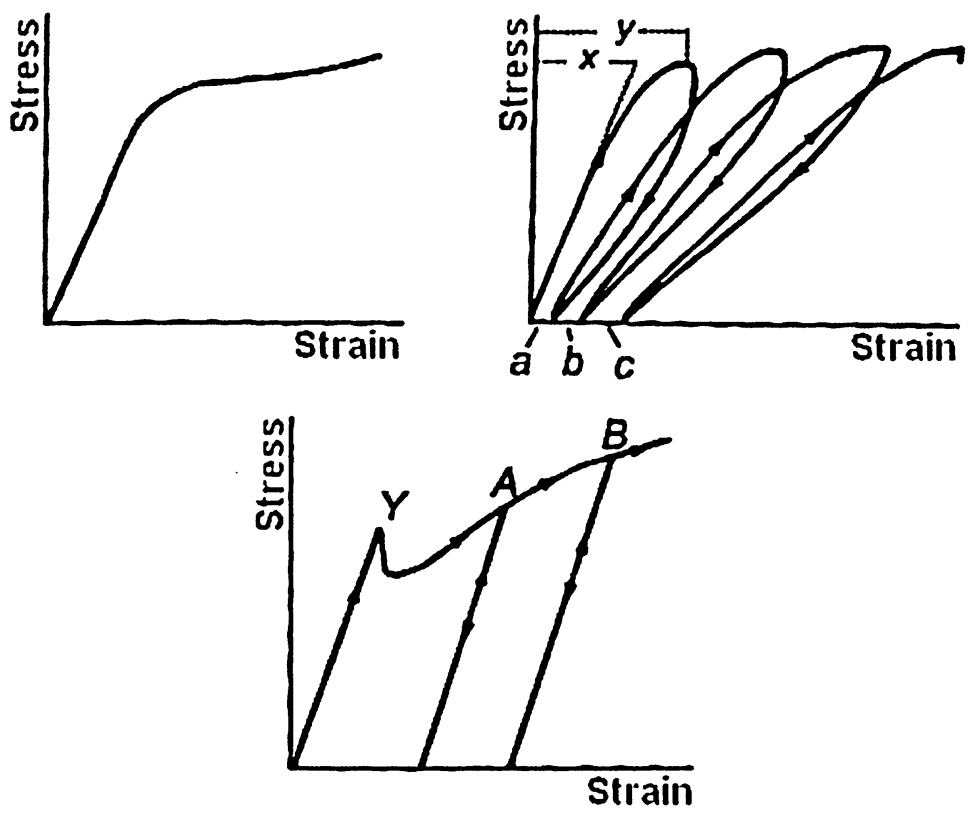

Fig. 2.4: (Top left) Stress-strain curve of bone loaded in a single pull to fracture. (Top right) A bone specimen loaded and unloaded three times and then loaded to failure. (Bottom) Mild steel loaded beyond the yield point $Y$ and then unloaded twice, at $A$ and $B$ (Currey [12]).

\subsubsection{Modes of Loading}

Bone is stronger when loaded in compression than loaded in tension. In general, bending strength values are greater than those in tension and compression. If the stress at failure is calculated using beam equations, which do not evaluate the effect of post-yield deformation, bending strength for bones are $50 \%$ greater than the corresponding tensile strengths [15]. The mode of compressive fracture is rather different from tensile failure. The loaddeformation curve of specimens tested under compression show linear portion followed by a 
short yield region, the load soon dropping slightly and then continuing at roughly the same level until the specimen finally breaks up. This final breakup may occur only after considerable deformation. Yield stress is higher in compression than in tension (see Table 2.3). Yielding and deformation under compression is microscopically quite different from yielding under tension. Under compression, the shear lines show a strong tendency to initiate at large stress raisers such as blood channels, while this is not always the case for the small microcracks generated under tensile loading [3].

Bone undergoes failure when subjected to tensile loads. However, if it is loaded in bending the bone may well start to show little fracture on the compression side before the fatal crack starts on the tensile side. Fig. 2.5 compares bone load-deformation responses subjected to tensile and compressive loads.

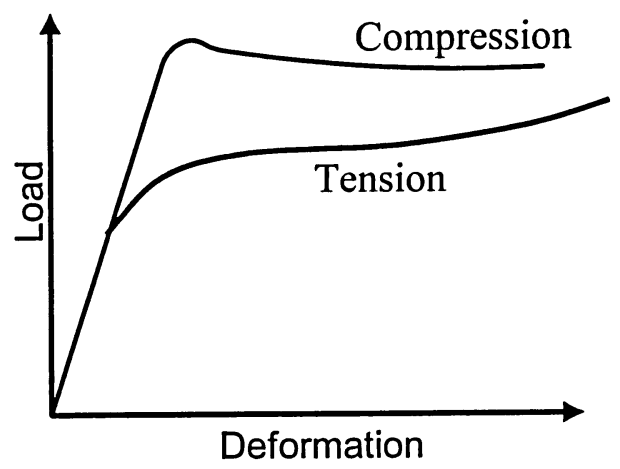

Fig. 2.5: Load-deformation curve for cortical bones under tension and compression [2].

\subsubsection{Microstructure}

Carter et al. [16] examined the relations between the mechanical properties and the structure of embalmed human cortical bone specimens. They demonstrated a negative correlation between ultimate tensile strength and the percentage of osteons in the specimen fracture surface. It was also found that both the percentage of osteons on the fracture surface and the number of osteons $/ \mathrm{mm}^{2}$ affect the hardness of bone structure. The percentage of osteons on the specimen fracture surface also affected the compressive strength of bone. Carter et al. [16] also found a positive correlation between bone compressive strength and the percentage of osteons in the specimen break area. Fig. 2.6 presents a typical microstructure found in Haversian system cortical bone. 


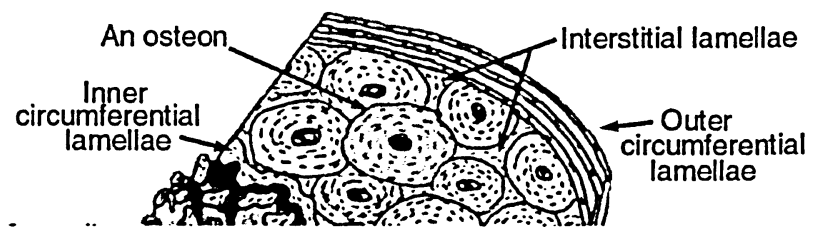

Fig. 2.6: Osteon (Haversian system) quantity and density and its ratio to interstitial lamellae and primary bone have a great effect on the mechanical properties of cortical bone.

\subsubsection{Orientation Effect}

Properties of bone considerably depend on stiffness measured along and perpendicular to the longitudinal axis of the bone. Values of stiffness measured along the bone longitudinal axis were found to be 1.6 to 2.4 times greater than those measured in transverse direction. In particular, bone has a much higher strain to failure when it is loaded longitudinally rather than circumferentially [12]. The orientation effect is more important in bones with the Haversian system as usually the osteons are laid out along the long loading axis (see Fig. 2.1).

As discussed earlier, osteon lamellae in cortical bone have different collagen orientations. The orientation of collagen fibres is more influential on the bone mechanical properties than the rate of mineralization (remodelled bone is usually less mineralized than primary bone). To determine the predominant orientation of the collagen in the specimen sections with respect to the long axis of the bone, Riggs et al. [17] used circularly polarized light images of bone structures. They found that collagen fibres arranged transversely showed a lower Young's modulus as compared with the collagen fibres which were longitudinally arranged when tested under tension and compression. Fig. 2.7 presents a typical Haversian osteon in cortical bone consisting of many lamellae (layers) with different collagen fibre orientations. 


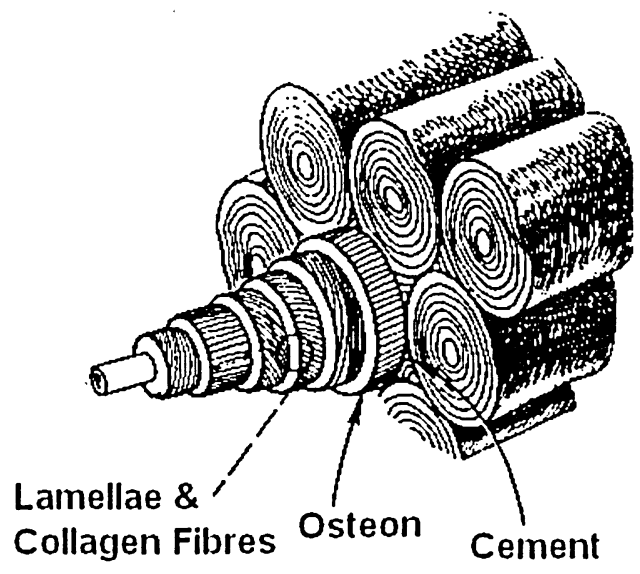

Line

Fig. 2.7: Haversian osteons consist of layered lamellae with different collagen orientations.

\subsubsection{Effect of Mineral Composition}

The mineral composition of bone structure largely influences the mechanical properties of cortical bone. The reason is that minerals (hydroxyapetite) possess a high Young's modulus of $110 \mathrm{GPa}$ (five times greater than Young's modulus of cortical bone) which drastically affect the mechanical properties of bone. Kotha et al. [18] found that the elastic modulus of bone increases with an increase in the aspect ratio of mineral platelets (length/thickness) and with an increase in organic matrix elastic modulus. They also found that the elastic modulus of bone tissue increases with an increase in the volume fraction of bone mineral.

\subsubsection{Effect of Stress Raisers}

Bone structure consists of potential stress raisers such as cavities for blood vessels, osteocyte lacunae and canaliculi. Fig. 2.8 shows some of the stress raisers in cortical bone structure. Currey [19] showed that the stress-raising effects of the flattened osteocytes could be as great as $7 \mathrm{~S}$, where $\mathrm{S}$ is general level of stress. Blood channels are the largest potential stress concentrators. However, they run predominantly in a very gently spiralling course through long bones, at quite a small angle to the bone longitudinal axis. Canaliculi spread right through bone tissue at all angles and, therefore, theoretically must produce stress concentrations. However, they are very small, about $0.3 \mu \mathrm{m}$ in diameter, and so may not be effective in helping cracks to spread. 


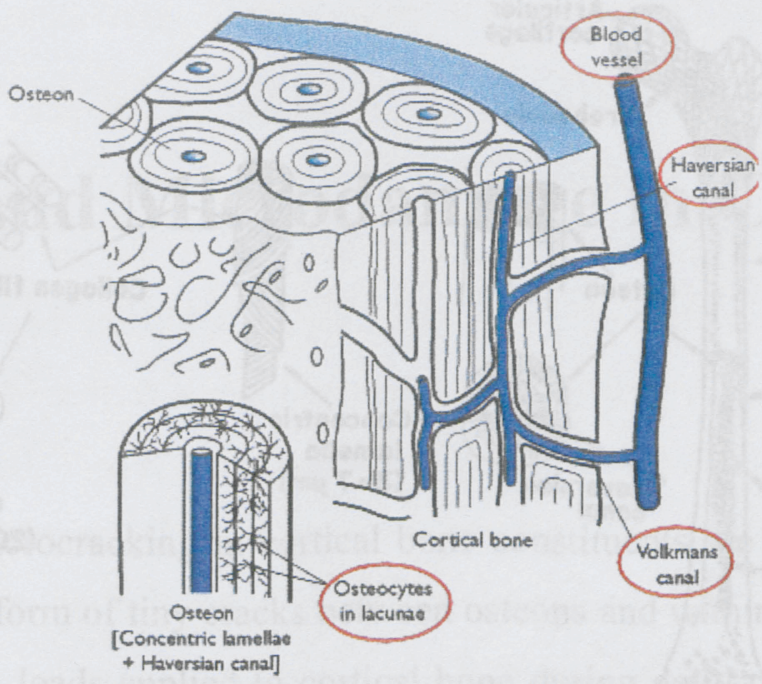

Fig. 2.8: Some of the stress raisers in cortical bone (circled).

\subsection{Bone as a Natural Composite}

A material like bone, with a strong hierarchical structure, consists of phase changes and material/mineral direction changes over the length of the cortical bone shaft, analogous with composite materials. Such structure contains several microcracks over constituents of bone structure.

Bone micro-scale structure consists of the collagen as matrix in which the mineral fibres are embedded. The structure of the bone can also be characterized with the collagen fibrils, with their associated minerals, as fibres, each more or less loosely connected to its neighbouring fibres, all of them making lamellae. The lamellae are separated from their neighbours by a change in the predominant orientation of the collagen fibres and their associated plates. This discontinuity will be a line of weakness.

On a larger scale, the structure of bone is constructed from the osteons as fibres, again more or less loosely connected to each other by cement lines as matrix. Fig. 2.9 schematically illustrates the structure of bone and its features as a natural composite. 


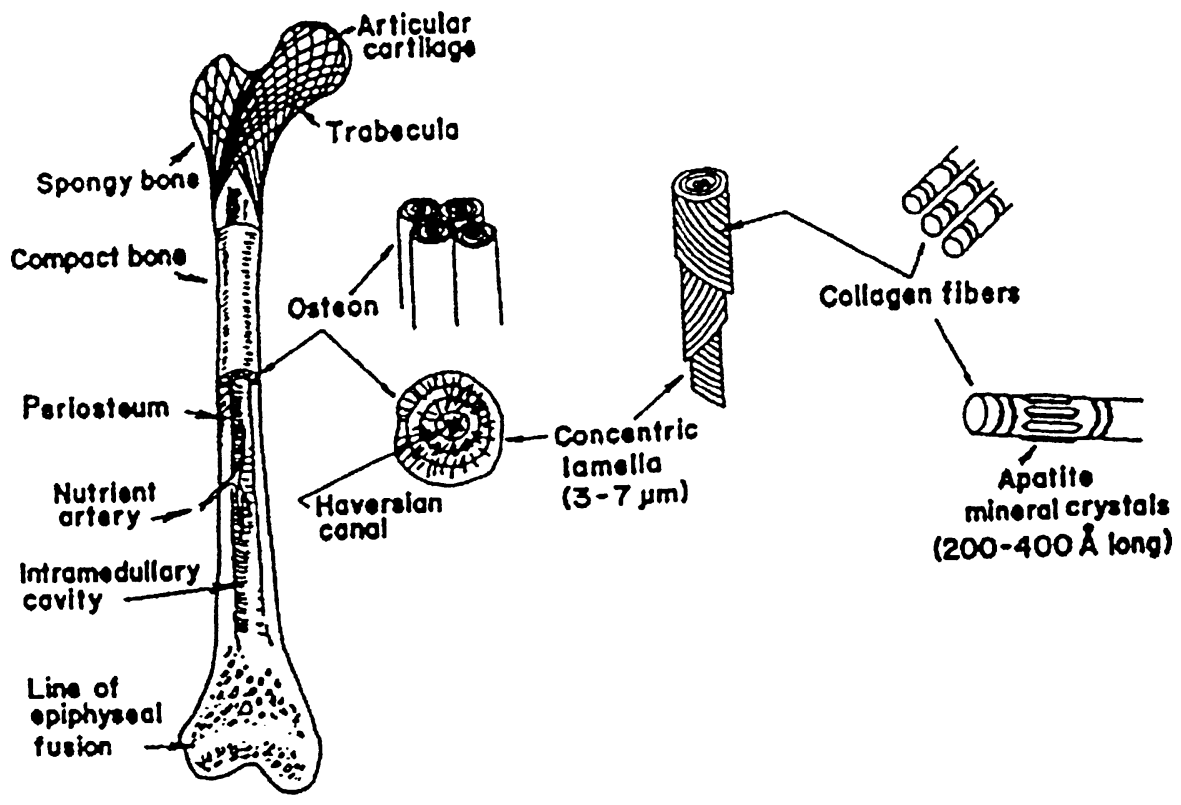

Fig. 2.9: Hierarchical levels of structure in a human femur [11]. 


\section{Chapter 3}

\section{Fatigue and Microdamage in Cortical}

\section{Bone}

Fatigue damage and microcracking in cortical bone constituents are inevitable phenomena. Damage appears in the form of tiny cracks between osteons and within cement lines. Osteons take most physiological loads applied to cortical bone during daily physical activities. This very much resembles fatigue damage response in composites. Cortical bone is a natural composite material with secondary osteons as fibres and the interstitial bone as the matrix. The resistance of any material to fatigue failure is a function of its resistance to either or both the initiation and propagation of cracks. Osteonal bone can be compared to composite materials and to metals whereby discontinuities within the material (e.g. fibres, laminae, voids) may provide stress concentration sites for crack initiation, but they also serve as barriers to crack growth which may slow down or even halt crack propagation [20]. In this chapter the analogy of bone to composite materials and its effects on the fatigue response of cortical bone are further discussed.

\subsection{Microdamage Mechanisms in Cortical Bone}

The main difference between the behaviour of a metal and a bone is that in post-yield region, bone undergoes damage, in the form of microcracking reducing the stiffness of bone structure. Microcracks within interstitial bone are created during normal physiological activities. These tiny cracks in bone are too small to be dangerous, in their early stage [12]. Based on bone health, age, nutrition and other biological parameters, remodelling process can repair microcracks through on-time cell communications. 


\subsubsection{Microcracking Phenomenon}

Bone under daily physiological loads produces several microcracks each corresponding to the release of some energy. Such tiny cracks were observed by Currey and Brear [21] when they loaded bone samples under three-point-bending and immersed them in stain. They have also reported that the number of microcracks significantly increases when bone samples under tensile loading exceed yield point. As the specimen is further loaded into the post-yield region the microcracking becomes increasingly pervasive. Fig. 3.1 represents microcracks formed between osteons in cortical bone. Burr et al. [22], based on the results of their experiments concluded that microcrack accumulation impairs the mechanical properties of bone by reducing its elastic modulus (stiffness reduction). They also showed that there were significantly more microcracks in tensile cortices, but on average cracks were significantly longer in compressive cortices.

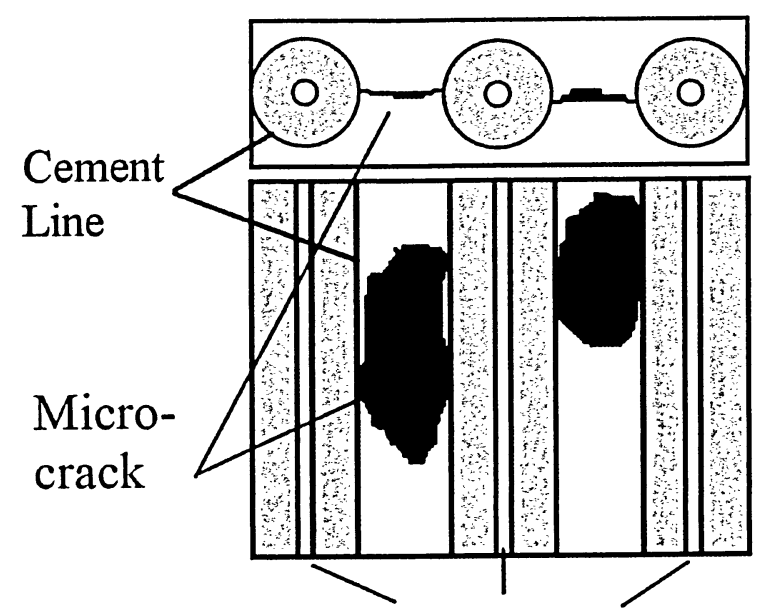

Osteons

Fig. 3.1: Schematic representation of microcrack arrested between the cement lines.

\subsubsection{The Mechanical Effects of Microcracking}

In post-yield region, individual microcracks do not get longer; instead, more and more of them appear. There is no really consistent pattern to where the cracks form. Sometimes they form near blood channels, and frequently little arrays of cracks are seen associated with osteocyte lacunae. In compression and shear the microcracks seem to form preferentially in the direction of lamellae, and also to follow around the cement lines of secondary osteons 
[23]. In bone, microcracks form rather easily and spread a little, but then require a considerably greater stress to spread further. This will explain the increase in strain to failure in stress-strain curve of bone and large flattened part of it. Fig. 3.2 shows an example of cyclic stress-strain hysteresis loops for human cortical bone. This figure shows that as the number of cycles increases, hysteresis loops shows a decay in stiffness (loss is secant modulus) of bone structure.

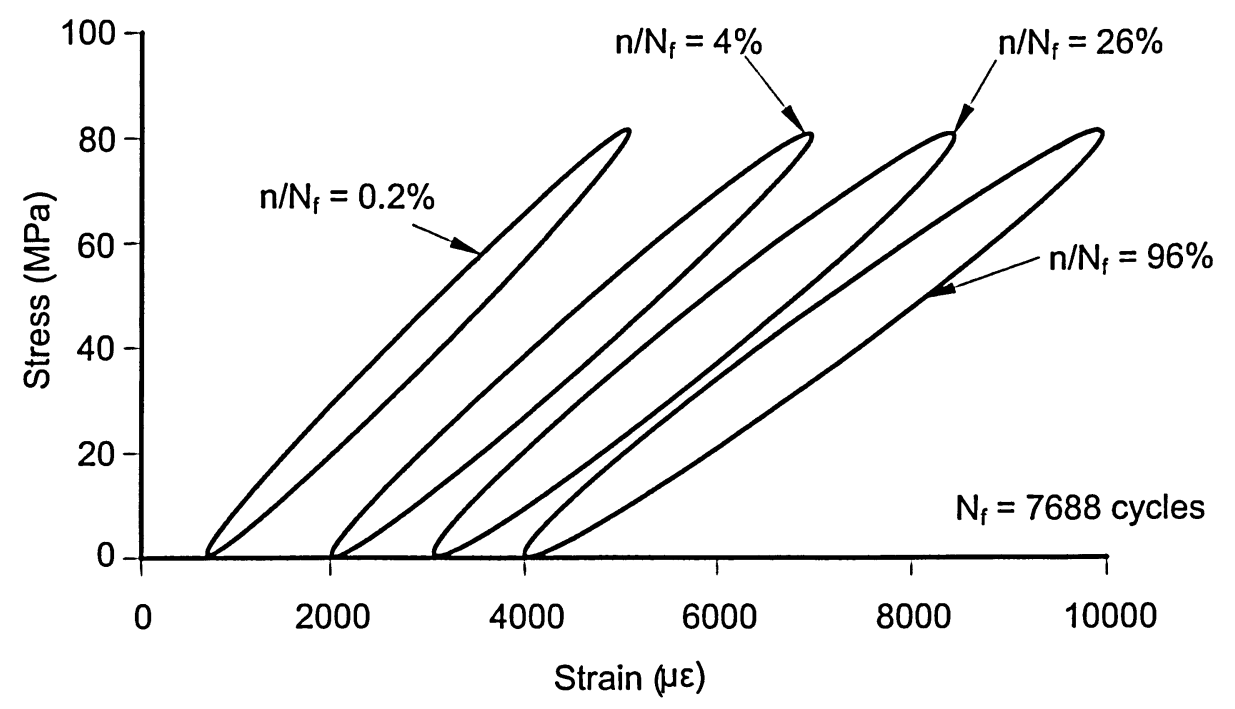

Fig. 3.2: A stress-strain hysteresis loops of human cortical bone tested under stress-controlled condition (Pattin et al. [24]).

Zioupos and Casinos [25] pointed out that bone, due to its specific tomographic structure impedes a crack to grow appreciably in length. This is in contrast to the nature of brittle materials, in which the resistance to crack growth decreases as cracks get longer resulting in released strain energy. More damage accumulation and progress as a consequence of forming of microcracks in bone structure leads to a higher strain energy and stiffness reduction in bone structure becomes more pronounced.

\subsubsection{Microcracking of Bone as a Composite Material}

In a well designed composite material any crack, in its early stage of growth, is forced to change its direction, or to pass into a void that makes it blunt-ended, or to give up the strain energy, as a driving force is required to pull strong fibres out of matrices [12]. Fig. 3.3 presents crack growth morphology in different materials. In brittle material, once crack starts 
to grow it is unimpeded (Fig. 3.3A). The crack passes into a set of layers in which the crack propagation along two inclined directions is possible. The two solid lines from the end of the crack (Fig. 3.3B) represent cracks at two different depths. This mechanism of crack stopping is found in tooth enamel. Fig. 3.3C represents cracked material with voids (or often weak interfaced) causing a crack running into them with difficulty in further propagation, as the crack tip is blunted. Fig. 3.3D shows fibres embedded in the material. As the crack advances it has to break through the strong fibres. It also has to extend energy overcoming frictional forces in pulling the fibres out of composite material.
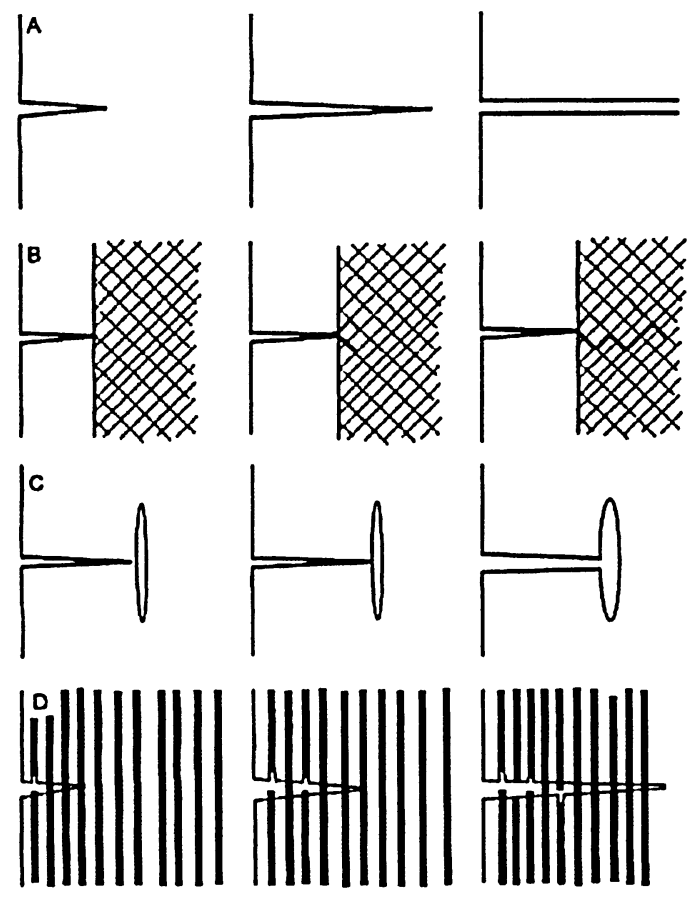

Fig. 3.3: Crack growth in different materials (image from Currey [12]).

Under cyclic loading, bone behaves like a composite material. Fatigue loading results in matrix microdamage and concurrent degradation of bone stiffness [6]. Fatigue and resulting microdamage occur readily even at the modest stresses or strains to which bone is subjected to its habitual physiological loads. However, at low stress/strain levels, microdamage does not readily progress to failure. Unlike synthetic engineering materials, bone is capable of microscopic repair at the level of matrix microdamage, through a coordinated multi-cellular process (i.e., bone remodelling) which can remove and replace damage foci before they coalesce and lead to catastrophic failure of bone. 


\subsubsection{Osteon Pullout}

Osteon pullout is thought to occur when the tensile strength of an osteon exceeds the shear strength at its cement line or interlamellar interfaces, causing the osteon to debond and bridge a propagating crack, and then be pulled out of the fracture surface in a telescoping fashion. This phenomenon has been shown in Fig. 3.4 in cortical bone samples from various species fractured in tension or bending at slow strain rates.

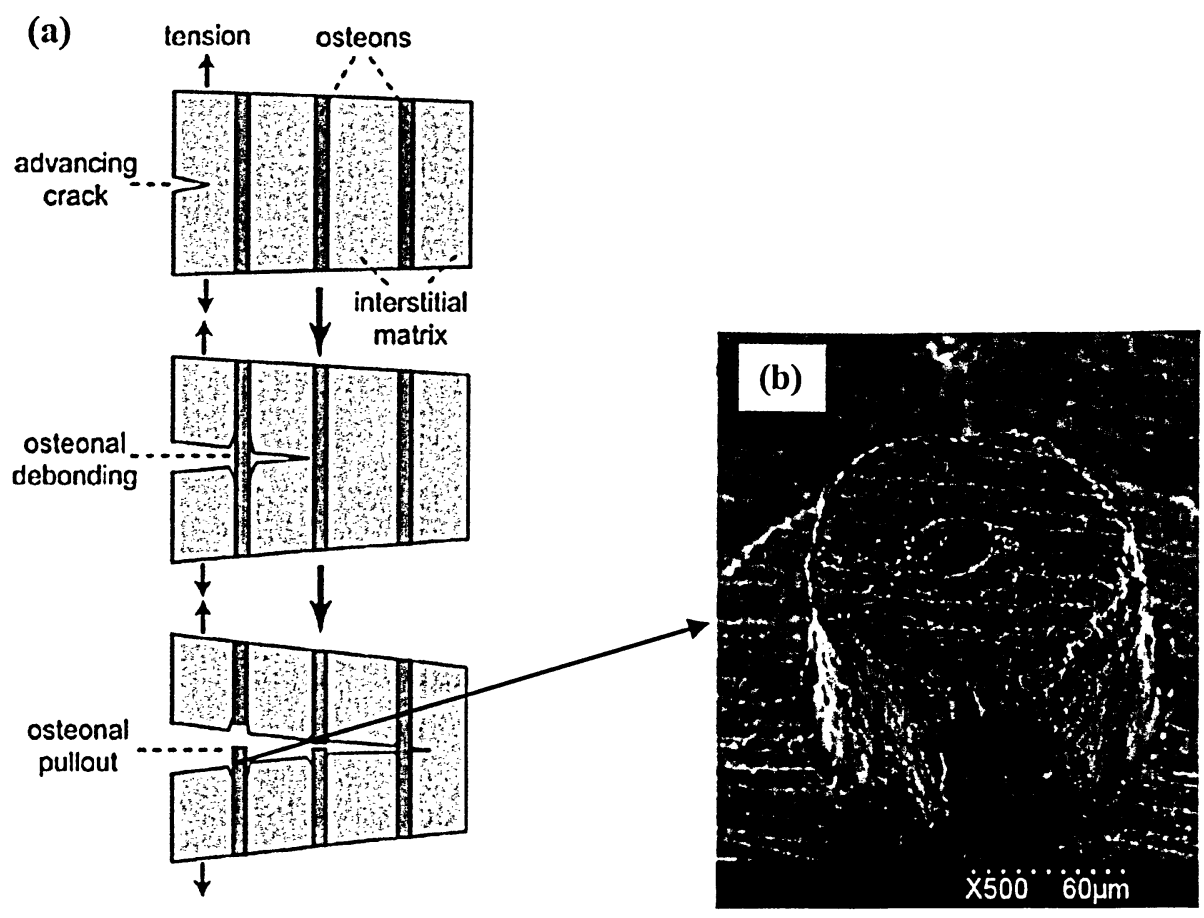

Fig. 3.4: a) Schematic diagram illustrating the mechanism of osteon pullout [26]. Tensile loads produce a crack that advances transversely across intact osteons embedded in the interstitial matrix. The matrix shears from the osteon cement line, resulting in debonding and osteonal crack bridging. Eventually the bridging osteons fail in tension b) image of an osteon pulled out from bone tissue.

\subsubsection{Remodelling and Repairing Process of Microcracks}

Haversian bones are weaker (less stiff) than the primary osteon bone, particularly under tensile loads. Their relatively high toughness makes them potentially capable to arrest cracks within cement lines [12]. When bone is loaded heavily, it undergoes more remodelling process. Martin and Burr [27] presented a theory stating that cyclic stress initiates 
remodelling in compact bone. The theory is based on the observation that the lamellar structure of osteons arrests and traps microcracks in bone produced by cyclic loading.

Once a microcrack approaches an osteon, it enters the cement line and follows it for some distance. Alternatively, the crack may pass through the cement line to follow an interface between lamellae within an osteon. In either situation, the crack would substantially debond or separate the Haversian canal from the surrounding bone. It was hypothesized that this osteon debonding causes physiologic changes to cells within the Haversian canal which lead to the repair of the damaged regions. The repair is affected by a new osteonal tunnelling and refilling process emanating from the wall of the Haversian canal. This repair prevents the accumulation of microdamage due to cyclic loading and increases the fatigue life of cortical bone [12].

\subsection{Parameters Affecting Fatigue Damage of Human Cortical Bone}

The damage process in cortical bone is very much dependent on (i) physiological loads applied to the bone and resistance of bone micro-constituents against applied loads and (ii) biological parameters such as bone density, age and osteon volume fraction. An understanding of these parameters in cortical bone further helps to realistically analyze damage of cortical bone subjected to cyclic loading conditions.

\subsubsection{Stress Amplitude}

Swanson et al. [28] examined specimens taken from 22 human cadaver femora, with ages at death ranging from 35 to 98 years. Specimens were taken from the mid-third of the bone along the longitudinal axis of bone shaft. Fig. 3.5 shows a schematic drawing of bone specimens tested by Swanson et al. [28]. 


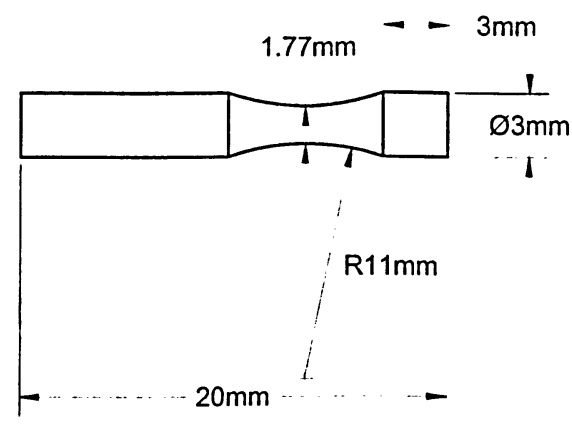

Fig. 3.5: Dimensions of bone specimens tested by Swanson et al. [28]

Throughout the experiment, the surface of the specimens were kept moist by contact with a wick supplied from a reservoir of Ringer's solution. The importance of keeping the bone specimens wet throughout the tests have been repeatedly emphasised by almost all researchers in order to resemble the in vivo condition as close as possible.

The amplitude of the stress was taken, in the usual way, as being the bending stress at the top and the bottom surfaces of the waisted portion. Swanson et al. [28] further discussed the importance of testing conditions such as bone dryness and surface treatment of samples. But more importantly their test results showed a logarithmic relation between the applied stress amplitude and the median fatigue life of bone specimens. This relation which is presented in Fig. 3.6 can be seen in the work of many other researchers.

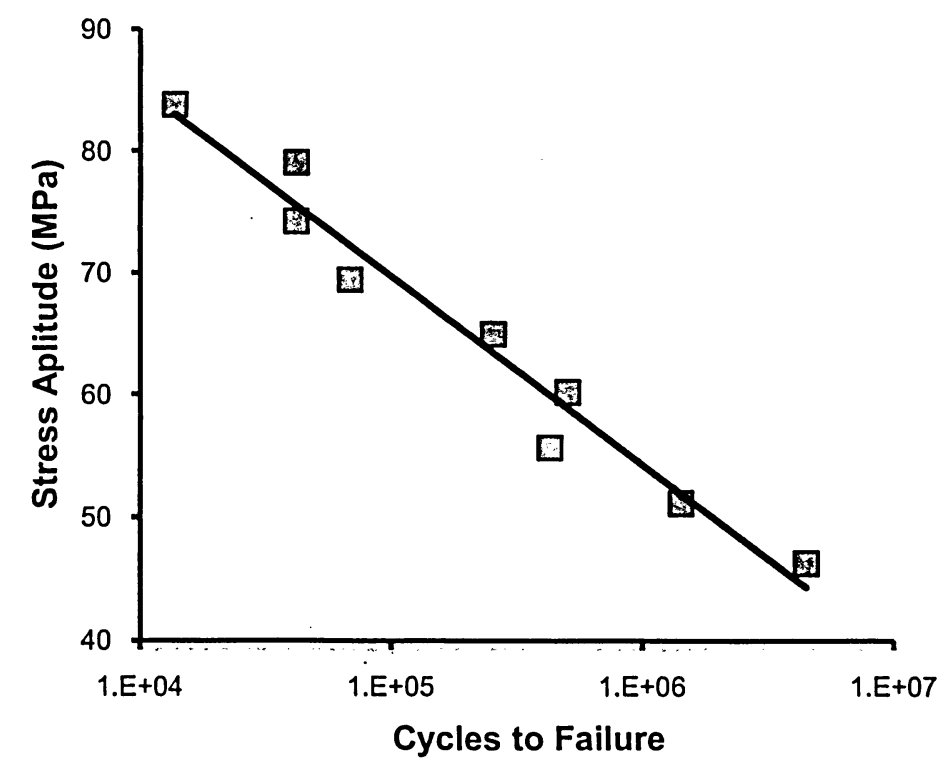

Fig. 3.6: The median s-n curve for all cortical bone samples tested by Swanson et al. [28]. 


\subsubsection{Density of Micro-constituents}

Shaffler et al. [29] studied compact bone microdamage from human femoral cortical bone under a wide age range. They obtained femoral diaphyses from 25 individuals ( 16 males, 1673 years old; 9 females, 28-63 years old). All specimens were taken from individuals who had died acutely and had no history of chronic illness. They observed that the vast majority of the microcracks were found running within interstitial bone tissue. Interstitial bone is the oldest tissue, comprised of fragments of osteonal and other lamellar bone fragments from previous modeling and remodelling cycles. Interstitial bone is less fracture resistant than osteonal tissue. They showed that cement lines are less mineralized than lamellar bone. As such they present weak interfaces within the bone microstructure, and have been postulated to serve as potential sites for both crack initiation and as low stiffness interfaces in bone structure. Their results showed that the density of bone micro-constituents such as osteons and interstitial bone greatly affected the fatigue life of cortical bone samples.

\subsubsection{Loading Type}

George and Vashishth [30] investigated fatigue damage development in cortical bone in vitro under different mechanical components of physiological loading including tension, compression, and torsion. During each test, they continuously collected stress and strain data to monitor and statistically determine the occurrence of the primary (stage I), secondary (stage II), and tertiary (stage III) phases associated with fatigue failure of bone. The tensile group demonstrated Mode I cracking and the three classic stages of fatigue suggesting a low crack initiation threshold, steady crack propagation and final failure by coalescence of microcracks.

In contrast, the compressive group displayed Mode II cracking and a two-stage fatigue behaviour suggesting a high crack initiation threshold followed by a sudden fracture. The torsion group also displayed a two-stage fatigue profile but demonstrated extensive damage from mixed mode (Modes II and III) microcracking and predominant time-dependant damage.

The loading histories for the three groups of specimens that George and Vashishth [30] have tested included: 
I. Zero to Tension at $50 \%$ of the Ultimate Tensile Strength (UTS) $=0 \mathrm{~T}$

II. Zero to Compression at $50 \%$ of Ultimate Compressive Strength (UCS) $=0 \mathrm{C}$

III. Zero to Torsion at $30 \%$ Ultimate Shear Strength (USS) $=0 \mathrm{R}$

Stiffness degradation profiles between above three groups are shown in Fig. 3.7.

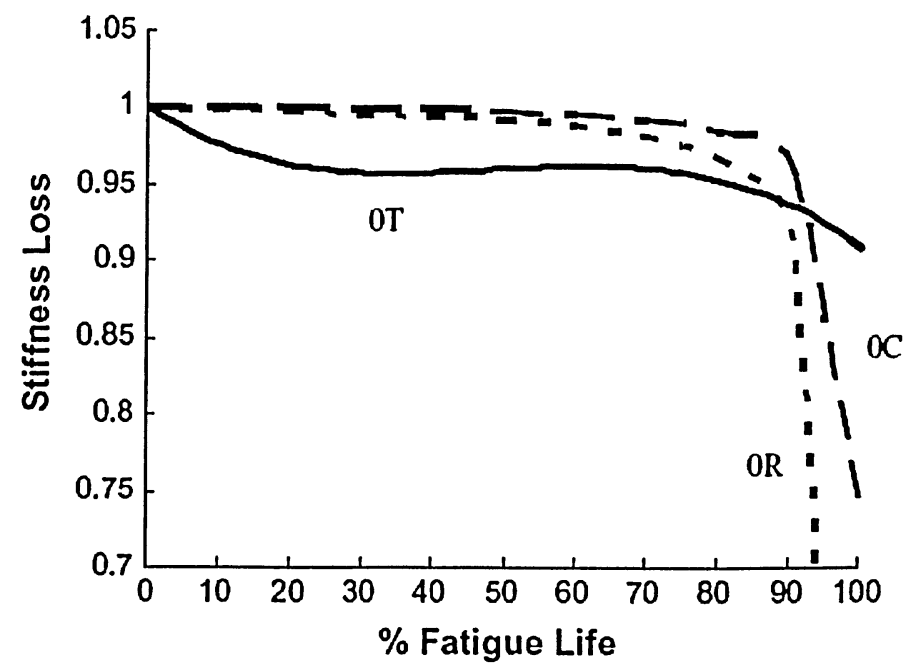

Fig. 3.7: Average stiffness loss profiles for specimens subjected to ZeroTension (0T), Zero-Compression (0C), and Zero-Torsion (0R) [30].

They also observed that compressive loading produced fewer but longer microcracks on cortical bone samples. Cracks in compression were therefore difficult to initiate but easy to propagate. They postulated that these microcracks were likely to result from the shearing of longitudinally oriented osteons under the action of mode II loading. In their studies the relatively weak cement line interface between the osteon and interstitial matrix provided a weak interface where microcracks under shear were easily produced.

Furthermore, the relatively smoother oblique fracture profile of $0 \mathrm{C}$ specimens (Fig. 3.8b) corresponded to a shear-dominated compressive fatigue failure. Crack orientation has been shown to be an important factor in determining mechanical properties and damage accumulation. Transverse microcracks, however, showed marginal effect on compressive stiffness due to crack closure that occurred during the compressive loading.

George and Vashishth [30] have also demonstrated that $0 \mathrm{R}$ specimens underwent the greatest stiffness loss (73\%) and, therefore, had the most aggressive damage mechanism. In contrast 
to the $0 \mathrm{C}$ group, the $0 \mathrm{R}$ group demonstrated spiral fracture revealing mixed mode failure (Fig. 3.8c).

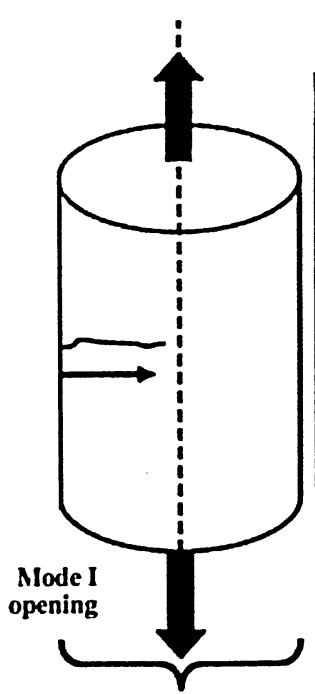

(a) Tension

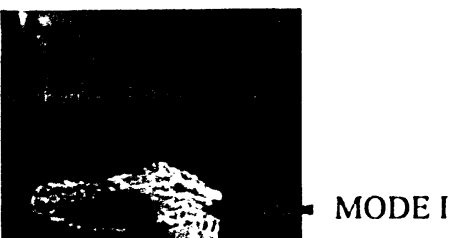

(a)

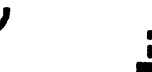

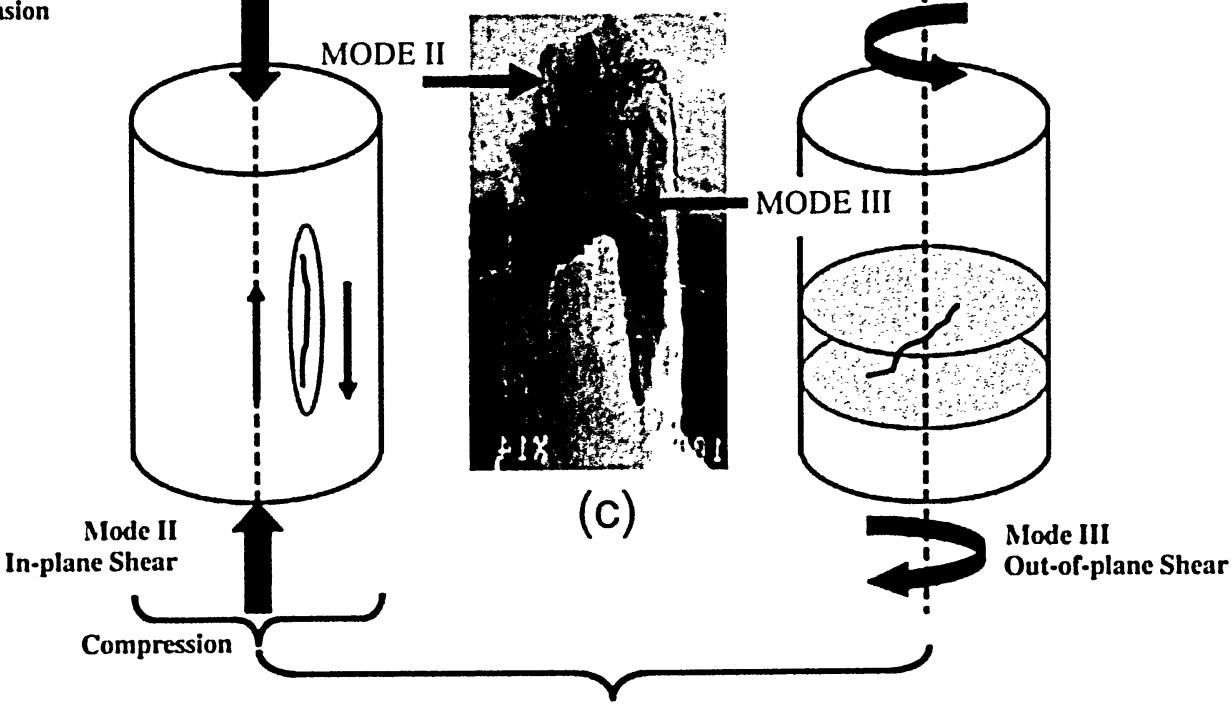

(c) Torsion

Fig. 3.8: Fracture surfaces of cortical bone specimens subjected to cyclic loading along with the schematic representation of micro-crack development in specimens subjected to Zero-Tension (Mode I) (a), Zero-Compression (Mode II) (b), and Zero-Torsion (Modes II \& III) (c) loading [30].

\subsubsection{Microstructure and Remodelling}

Carter et al. [16] examined the relation between fatigue life and the microscopic structure of adult human cortical bone. Bone samples were machined from unembalmed tibias and 
consisted principally of secondary osteons, osteon remnants, and interstitial bone. After flexural fatigue tests, frozen cross sections of the specimens were taken to examine fracture surface of cortical bone. Using enlarged photomicrographs the area of the section representing Haversian canals, resorption areas, and of the blood vessels was determined and subtracted from the total area to yield a "corrected break area". Significant positive correlations were found between fatigue life and per cent of osteonal area and fragments in the corrected break area.

\subsubsection{Age}

Diab et al. [31] demonstrated that young donors ( $38 \pm 9$ years) had a four-fold longer fatigue life and formed more diffuse damage (scattered microcracks) than the older donors ( $82 \pm 5$ years). In contrast, old donors had a shorter fatigue life and formed more linear microcracks than the younger donors. Linear microcracks were longer in older than in younger donors and were associated with weak lamellar interfaces. Areas of diffuse damage were, however, larger in younger than in older donors. Their findings showed that the tendency of bone to form a particular damage morphology is subject to change with age and that the propensity of young donors to form diffuse damage over inter-lamellae linear microcracks plays a critical role in the ability of bone to dissipate energy and resist a catastrophic fracture. Age-related changes in damage morphology may therefore be an important contributor to the increased bone fragility in the elderly.

Microdamage accumulation, in vivo, depends on the imbalance between the rate at which the remodelling process repairs fatigue damage and the rate at which microdamage is initiated. Microdamage occurs in the form of linear microcracks and diffuse damage. Linear microcracks form under compressive loading in the interstitial bone tissue and stop at osteonal boundaries. In contrast, diffuse damage forms under tensile loading as submicroscopic cracks in the interstitial bone.

Shaffler et al. [29] also found that the morphology of microcracks differed in young and old bone specimens. Microdamaged regions in the younger specimens often showed numerous small microcracks. Microdamage in older specimens typically appeared more sharply with 
less evidence of collateral damage. They suggested a strong, age-related increase in microcrack density in both males and females.

Diab et al. [31] concluded that the formation of diffuse damage occurred at an early stage in the fatigue life, while the formation of linear microcracks occurred towards the end of the fatigue life. The ability of cortical bone to compartmentalize damage morphology in different stages of fatigue damage, therefore, played a critical role in dissipating energy and resisting a catastrophic fracture. The bones donated by older donors showed a reduced tendency to form diffuse damage that dissipated energy. More significantly, older donor bones demonstrated a higher tendency to form linear microcracks that are associated with rapid crack propagation and catastrophic fracture in stage III. Fig. 3.9 shows decay in fatigue life of cortical bone as age increases. The modulus degradation curves were observed to be different for the younger and the older age groups as shown in Fig. 3.10. Old donors displayed the three phases of modulus loss. They underwent an immediate loss of stiffness in the primary phase followed by a secondary phase that was both short and associated with greater loss of stiffness over life cycles. In contrast, young donors showed a continuous drop in modulus degradation throughout the fatigue life. Furthermore, these specimens lost stiffness gradually in the primary phase, and displayed a longer secondary phase.

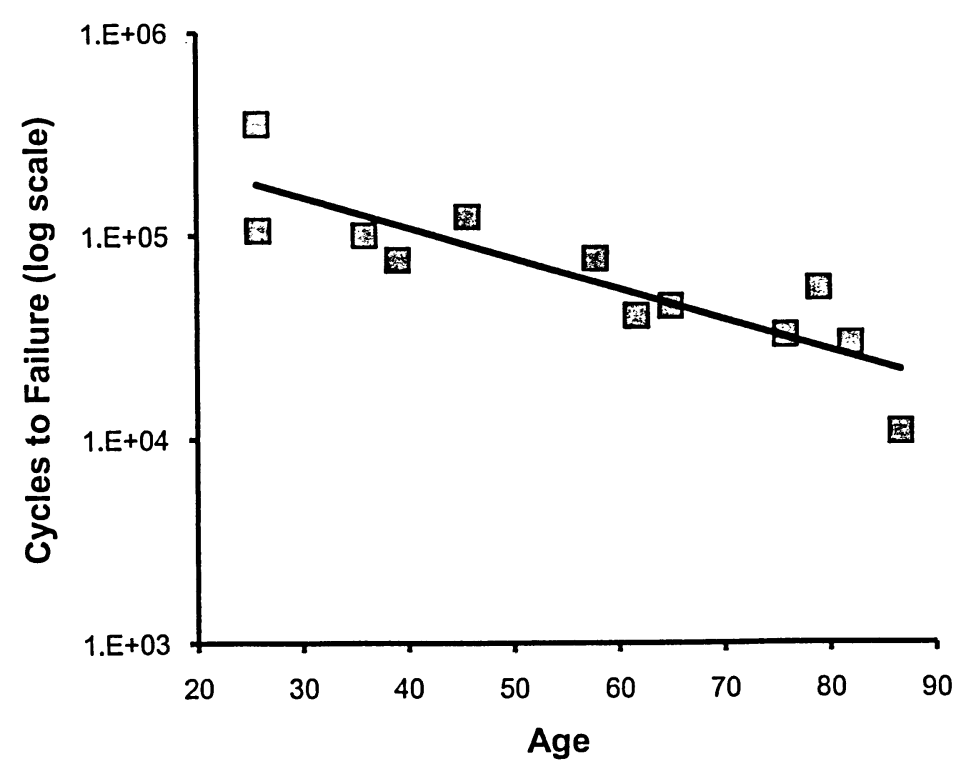

Fig. 3.9: Age as a function of life cycles to failure. Fatigue life exponentially decreases with age [31]. 


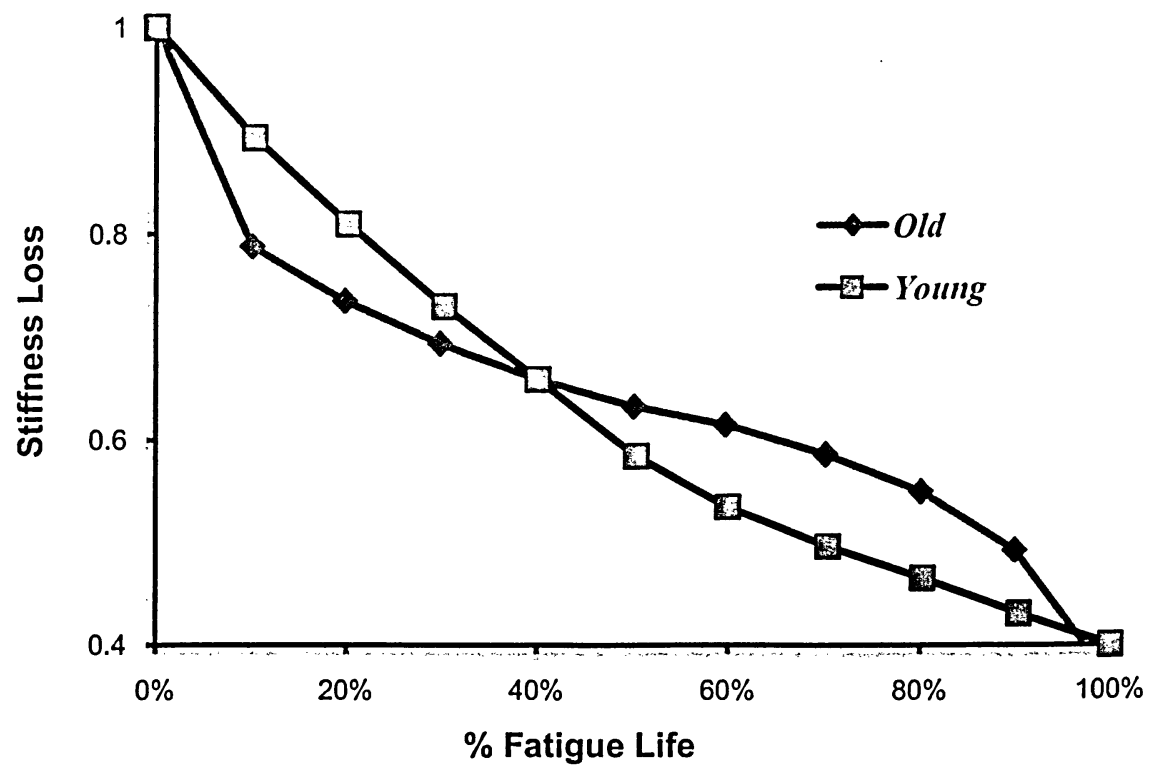

Fig. 3.10: Modulus degradation curves for young and old donors. Young donors display a continuous drop in stiffness, while old donors show three stages of fatigue damage [31]. 


\section{Chapter 4}

\section{Analysis and Modeling of Fatigue Damage in Human Cortical Bone}

\subsection{Introduction}

Cyclic loading of bone, as in all materials, leads to incremental failure through a process known as fatigue. This incremental failure process corresponds to the damage accumulation within structure and micro-constituents of bone. Mechanically, the accumulation of microdamage is correlated to loss of material stiffness as the fatigue cycles progress [6].

In order to determine the causes of repetitive stress injuries, the mechanism for fatigue damage accumulation in cortical bone is of great interest to researchers and clinicians. Investigations on the fatigue of structural materials and engineering composites are well established. However, the fatigue processes of bone are not as well understood. Cyclic loading of cortical bone produces noticeable damage in the form of microcracks which are thought to degrade mechanical integrity of bone structure [32].

In this chapter, fatigue damage of human cortical bone is analyzed and a mathematical model for predicting the damage behaviour is proposed. The fatigue damage analysis presented in this chapter aims to help researchers and clinicians to assess damage progress in microconstituents of bone structure at various stress magnitudes and different human age groups as the number of loading cycles increases. Fatigue damage behaviour in composite materials has been used as a fundamental concept to develop the proposed damage model for cortical bone. 


\subsubsection{Fatigue in Composite Materials}

In order to determine the overall damage in composite, contributions from individual constituents: the fibres, the matrix and the interface must be accounted for. The response of these constituents will greatly affect the mechanical properties of composite as a whole [33].

Rule of mixture is a fundamental principle in considering the effect of each constituent on the mechanical properties of a composite material. For stiffness of a continuous fibre-reinforced composite, the rule of mixture is given as:

$$
E_{c}=E_{m} V_{m}+E_{f} V_{f}
$$

where terms $E$ and $V$ corresponds to the modulus of elasticity (stiffness) and the volume fraction, respectively. Indices $f, m$ and $c$ denote the fibre component, the matrix component and the composite material, respectively. Dividing both sides of equation (4.1) by $E_{c}$ the rule of mixture is written as:

$$
1=\frac{E_{m} V_{m}}{E_{c}}+\frac{E_{f} V_{f}}{E_{c}}
$$

where $E_{m}, E_{f}$ : modulus of elasticity of matrix and fibres, respectively.

$V_{m}, V_{f}$ : volume fraction of matrix and fibres, respectively.

$E_{c}$ : initial secant modulus of composite material.

Equation (4.2) has been the backbone relation in damage analysis of composites. Ramakrishnan et al. [33] proposed a mathematical description of a stiffness-based damage model for fibre-reinforced glass-ceramic matrix composite. Based on terms of equation (4.2), the model predicts the drop in the composite stiffness as a function of fatigue cycles using mechanical properties of the composite constituents. Based on equation (4.2), one might suspect that one of the terms on the right of equation (4.2) might be due to the damage in the matrix and the other one due to the damage in fibres.

Stiffness loss as a damage index is characterized by $E / E_{0}$ which is the normalized secant modulus at any given loading cycle. $E_{0}$ is the maximum secant modulus for a given fatigue test which is approximately equal to the initial secant modulus measured prior to fatigue testing. To represent the accumulated damage at any load cycle, a new parameter can be defined based on stiffness loss as: 


$$
D=1-\frac{E}{E_{o}}
$$

where $D$ is the accumulated damage in fatigue loading. $D$ is zero at the beginning of fatigue life (no damage state) and increases to a maximum value of unity at the fracture point.

Assume that the stiffness $\left(E / E_{0}\right)$ of a specimen prior to damage is unity. Upon loading of specimen the stiffness of material is reduced and $\left(E / E_{0}\right)$ falls less than unity. Drop in stiffness of entire composite corresponds to $(1-D)$. Similarly, if drop in stiffness of both matrix and fibre phases, respectively correspond to $\left(1-D_{m}\right)$ and $\left(1-D_{f}\right)$, equation (4.4) is written as:

$$
(\mathbf{1}-\boldsymbol{D})=\frac{E_{m} V_{m}}{E_{c}}\left(\mathbf{1}-\boldsymbol{D}_{m}\right)+\frac{E_{f} V_{f}}{E_{c}}\left(\mathbf{1}-\boldsymbol{D}_{f}\right)
$$

Above equation presents the relation between the damage in composite and the damage in its constituents. Note that when there is no damage $\left(D, D_{m}\right.$ and $D_{f}$ are equal to 0$)$ and equation (4.4) will turn back into equation (4.2). Equation (4.4) can be rephrased as:

$$
D=1-\left\{\left[\frac{E_{m} V_{m}}{E_{c}}+\frac{E_{f} V_{f}}{E_{c}}\right]-\left(\frac{E_{m} V_{m}}{E_{c}} D_{m}+\frac{E_{f} V_{f}}{E_{c}} D_{f}\right)\right\}
$$

Therefore the fatigue damage equation in a composite material is yielded as:

$$
D=\frac{E_{m} V_{m}}{E_{c}} D_{m}+\frac{E_{f} V_{f}}{E_{c}} D_{f}
$$

where $D_{m}$ and $D_{f}$ are damage functions describing fatigue damage in matrix and fibres respectively. $D_{f}$ and $D_{m}$ have values that range from 0 , in an undamaged state, to unity, at failure point. It is assumed that at a time just prior to final failure, the matrix is entirely degraded and the fibres are degraded up to a point where the composite can no longer withstand the applied load.

\subsubsection{Fatigue of Cortical Bone as a Composite Material}

Cortical bone can be modeled as a fibre-matrix composite where fibres are complete osteons and the matrix is the interstitial bone (see Fig. 4.1). 


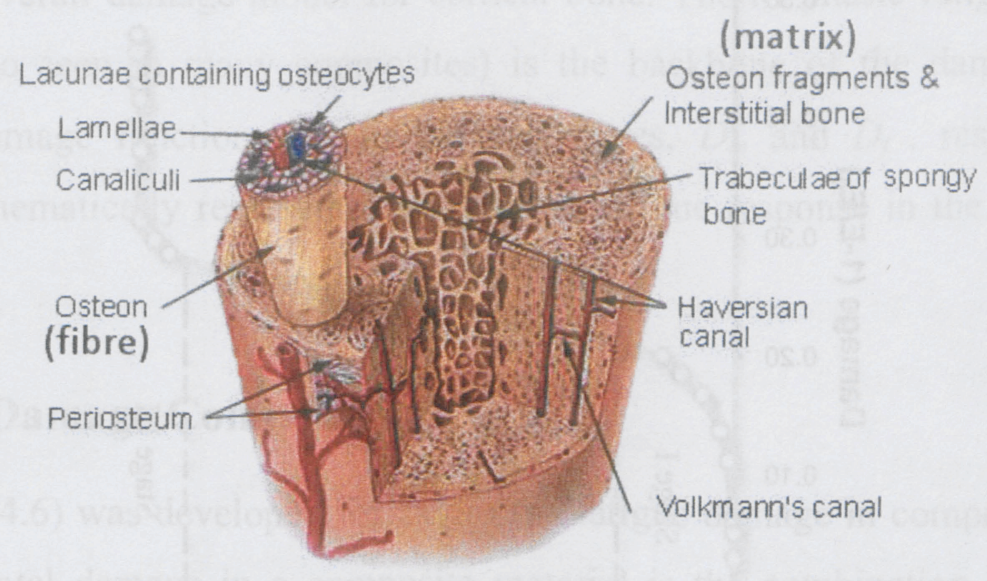

Fig. 4.1: Secondary osteons (Haversian system) type of cortical bone is shown. Osteons are considered to be acting like fibres embedded in interstitial bone as matrix in bone as a natural composite.

Considering other levels of bone structure (Fig. 2.9), fibres can also be taken as collagens embedded in bone cells as matrix. Even further down the structural hierarchy of bones one can assume fibres to be the minerals, with very high stiffness, as fibres embedded in collagen as matrix. At these micro-levels relating the fatigue behaviour of the bone to the mechanical and physiological properties of the fibre/matrix components is a very difficult subject matter. The difficulty with analysing bone as a composite material in its nano-size structure is mostly due to the lack of fully understood physical, structural and mechanical properties of constituents in bone hierarchical structure.

Fatigue response of human cortical bone has been reported to be analogues to that of a fibrereinforced composite material and has been characterized by three stages of fatigue damage under axial loading condition [12]. Fatigue diagram of composite materials show a tri-phasic failure behaviour as shown in Fig. 4.2. In this chapter cortical bone refers to human cortical bone unless noted otherwise. 


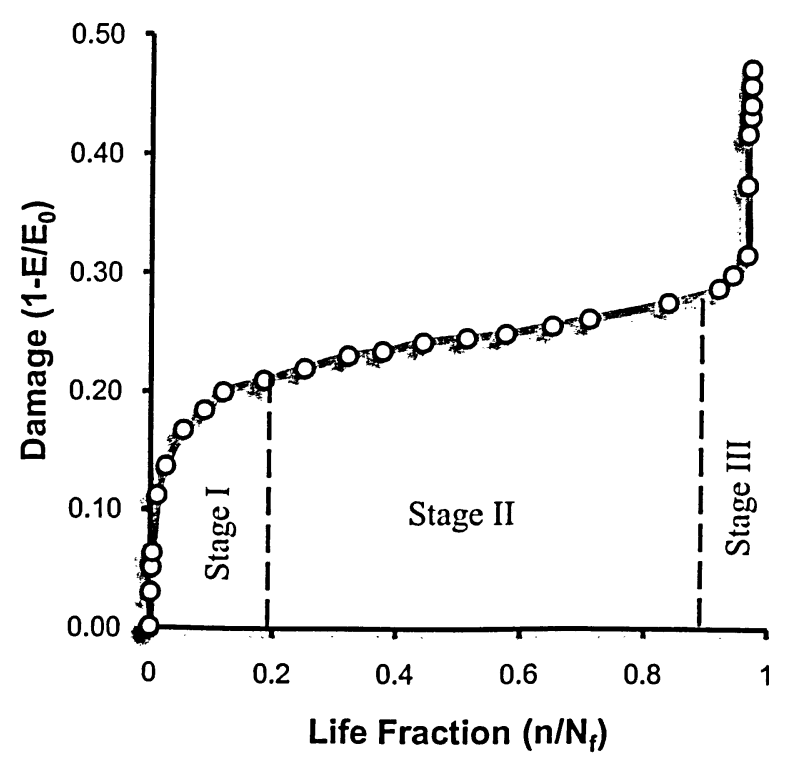

Fig. 4.2: A tri-stage fatigue behaviour seen in most composite materials as well as tensile fatigue of cortical bone (data from Pattin et al. [24]).

In stage I, a rapid but limited degradation in stiffness occurs which is associated with the initiation of microdamage. Stage II experiences the transition to a period of linear and gentle damage accumulation in which stiffness loss stabilizes. In the third stage, stiffness drops rapidly due to the coalescing of microcracks leading to rapid crack propagation and failure [24], [25], [31] and [32].

This type of fatigue response in cortical bone is representative of the fact that cortical bone acts like a fibre-reinforced composite under tensile fatigue loading. In the next section, a fatigue damage model is proposed for the prediction of fatigue response in cortical bone. This model is based on the fact that bone is a natural composite consists of osteons as fibres and interstitial bone as matrix.

\subsection{The Proposed Damage Model}

In this section, the steps in the development of the proposed fatigue damage model are presented. The proposed fatigue damage model has been developed for the cortical bone as a natural composite material considering secondary osteons as fibres and interstitial bone as matrix. Fig. 4.3 schematically represents the features of cortical bone as a natural composite. Separate damage functions were developed for fibres and matrix, respectively, and then 
integrated to an overall damage model for cortical bone. The tri-phasic fatigue response of cortical bone (also seen in many composites) is the backbone of the damage functions. Therefore, the damage functions for matrix and fibres, $D_{m}$ and $D_{f}$, respectively were developed to mathematically represent the tri-phasic fatigue response in the overall fatigue damage model.

\subsubsection{Fatigue Damage Components}

Earlier, equation (4.6) was developed for assess the fatigue damage in composites. One can assume that the total damage in a composite material is the combination of the damage accumulated in matrix and the damage incurred to the fibres. Replacing $E_{c}$ by $E_{0}$ (as both denote the initial secant modulus of composite) in equation (4.6) yields:

$$
D=\frac{E_{m} V_{m}}{E_{0}} D_{m}+\frac{E_{f} V_{f}}{E_{0}} D_{f}
$$

where $D_{m}$ and $D_{f}$ are damage to matrix and fibres, respectively. Equation (4.7) is the relation between the overall damage in cortical bone to damage in its constituents. Thus, in order to develop the damage model in cortical bone, damage functions $D_{m}$ and $D_{f}$ need to be mathematically described as functions of both mechanical and physiological parameters.

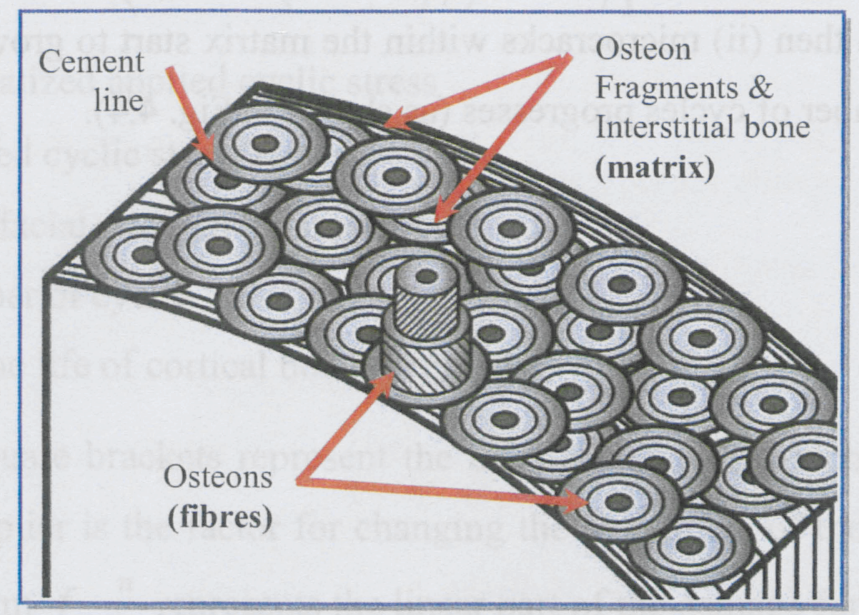

Fig. 4.3: Cortical bone as a composite material. Complete osteons are considered to act as fibres and interstitial bone and osteon fragments are taken as matrix. Cement lines separate the matrix from the osteon fibres. 
Considering bone as a natural composite material consisting of two phases of osteons and the interstitial bone around the osteons, $E_{m} V_{m} / E_{c}$ in equation (4.7) can be substituted by the following equation:

$$
E_{m} V_{m} / E_{0}=1-E_{f} V_{f} / E_{0}
$$

Additionally, in order to mathematically simplify the fatigue damage model and further reduce the number of input parameters $E_{f}^{*}$ is introduced as:

$$
E_{f}^{*}=E_{f} V_{f} / E_{0}
$$

By substituting equation (4.9) into equation (4.7) the short form of the proposed damage model for human cortical is achieved as:

$$
D=\left(1-E_{f}^{*}\right) D_{m}+E_{f}^{*} D_{f}
$$

Secant modulus and volume fraction of fibres (osteons) in cortical bone are primary terms of the parameter $E_{f}^{*}$ which are fully described later in this chapter.

\subsubsection{Damage Functions}

Damage in the matrix is characterized by two stages: (i) first microdamage within the interstitial bone initiates and grows non-linearly at a fast rate, but limited extent, with the number of cycles and then (ii) microcracks within the matrix start to grow linearly at a more gentle rate as the number of cycles progresses (as shown in Fig. 4.4). 


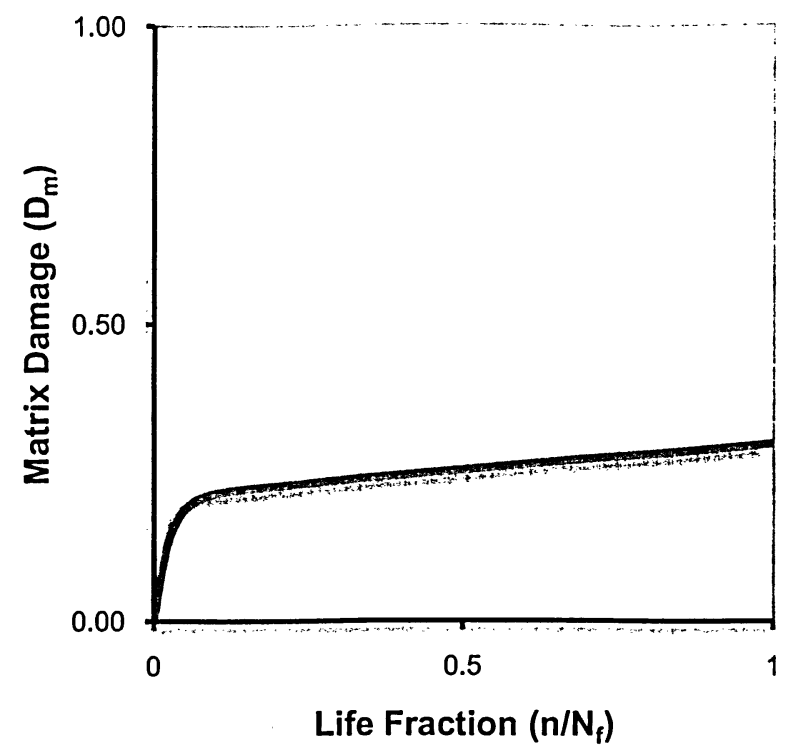

Fig. 4.4: A typical fatigue damage response in the matrix component of cortical bone.

In order to mathematically describe the response of matrix damage in cortical bone shown in Fig. 4.4, the following equation is proposed which presents the damage in matrix as the number of cycles increases:

$$
D_{m}=r \cdot\left[(1-f) \cdot\left(1-\exp \left(-\frac{\Delta \sigma}{2} \cdot \frac{n}{N_{f}}\right)\right)+f \cdot \frac{n}{N_{f}}\right]
$$

where $\quad r:$ normalized applied cyclic stress

$\Delta \sigma:$ applied cyclic stress range

$f: \quad$ interfacial strength parameter

$n$ : number of cycles

$N_{f}$ : fatigue life of cortical bone specimen

The terms in the square brackets represent the damage function which varies from zero to unity. The ' $r$ ' multiplier is the factor for changing the magnitude of the damage in different stress levels. The term $f \cdot \frac{n}{N_{f}}$ represents the linear part of the damage function with a slope of $f$. The first part of damage response (rapid stiffness loss) seen in Fig. 4.4 is best described by an exponential expression. Both parts of the matrix damage function are in terms of the ratio of numbers of cycles to fatigue life $\left(n / N_{f}\right)$ varying between zero to unity. 
The same approach has been taken to develop the damage function in fibres (osteons). However, damage accumulation in fibres follows a different trend and shape. Like many other composite materials, microcracks are arrested and stopped at the cement lines (interface between osteons and matrix) when fatigue loading begins. As the number of fatigue cycles progresses, microcracks slowly start to appear and linearly increase in osteons. The level of damage in matrix reaches a point that results in a sudden fracture of osteons (osteon pullouts). This evidence has been also reported by Zioupos and Casinos [25]. Fig. 4.5 presents damage progress in osteons as the number of fatigue cycles increases.

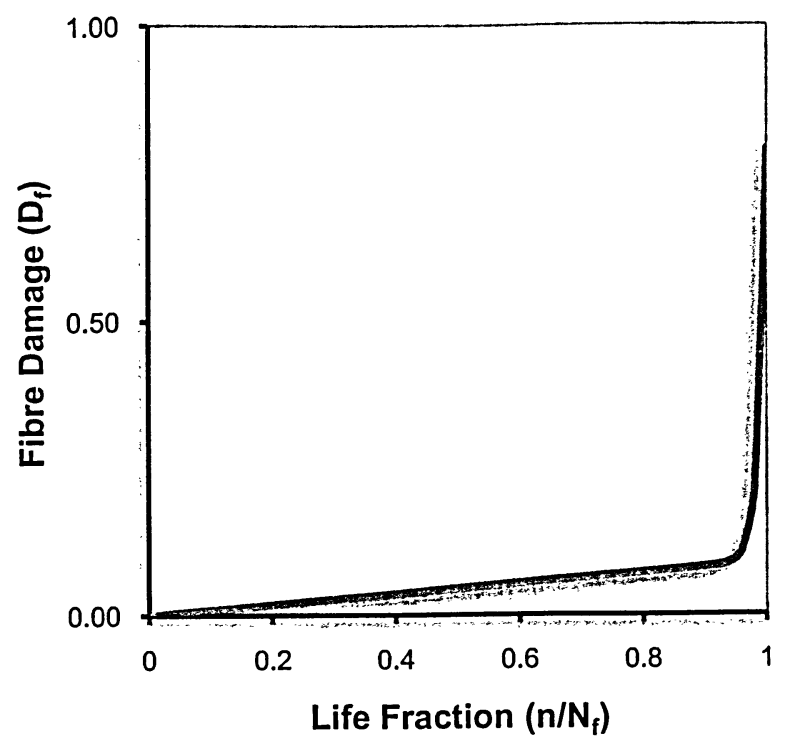

Fig. 4.5: Damage behaviour in fibres (osteons) component of cortical bone. Damage gently accumulates in a linear mode.

The following equation describes the damage accumulation in fibres (osteons) as the number of cycles progresses:

$$
D_{f}=r \cdot f \cdot \frac{n}{N_{f}}+(1-f) \cdot \exp \left(\Delta \sigma\left(\frac{n}{N_{f}}-1\right)\right)
$$

where $\quad r:$ normalized applied cyclic stress

$\Delta \sigma:$ applied cyclic stress range

$f$ : interfacial strength parameter

$n:$ number of cycles

$N_{f}$ : fatigue life of cortical bone specimen 
The first term $\left(r \cdot f \cdot \frac{n}{N_{f}}\right)$ in fibre damage function also represents the linear part of the damage accumulation. The second term which is an exponential term best describes the sudden increase in damage leading to fracture at the end of fatigue life of cortical bone.

\subsubsection{The Proposed Fatigue Damage Model}

The overall fatigue damage in cortical bone is the combined effect of the damage components in matrix and fibres. Equation (4.10) presents the amount of contribution of each damage component to the overall damage in cortical bone. Substituting equations (4.11) and (4.12) into equation (4.10) results in equation (4.13) for total damage assessment in human cortical bone.

$$
\begin{array}{r}
D=\left(1-E_{f}^{*}\right) \cdot r \cdot\left[(1-f) \cdot\left(1-\exp \left(-\frac{\Delta \sigma}{2} \cdot \frac{n}{N_{f}}\right)\right)+f \cdot \frac{n}{N_{f}}\right]+ \\
E_{f}^{*} \cdot\left[(1-f) \cdot \exp \left(\Delta \sigma \cdot\left(\frac{n}{N_{f}}-1\right)\right)+r \cdot f \cdot \frac{n}{N_{f}}\right]
\end{array}
$$

where $\quad r:$ normalized applied cyclic stress

$\Delta \sigma:$ applied cyclic stress range

$f:$ interfacial strength parameter

$E_{f}^{*}$ : ratio of osteon volume fraction and osteon modulus of elasticity to the initial modulus of elasticity of bone

$n$ : number of cycles

$N_{f}$ : fatigue life of cortical bone specimen

This model predicts the total fatigue damage accumulation in human cortical bone under a tensile cyclic loading. Integration of the damage in both constituents of fibres (osteons) and matrix (interstitial bone) and their result in an overall damage in cortical bone is presented in Fig. 4.6.

The proposed damage model includes no curve fitting constants. This makes the proposed model readily applicable to assess fatigue damage of cortical bone with no extra effort to determine coefficients other than mechanical properties of cortical bone and its constituents. 


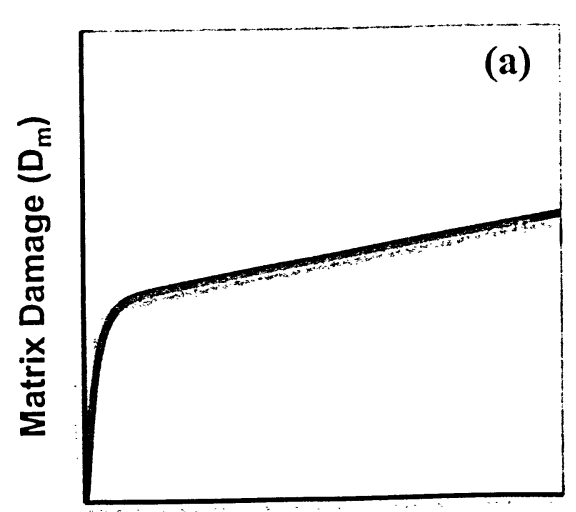

Life Fraction $\left(\mathrm{n} / \mathrm{N}_{\mathrm{f}}\right)$

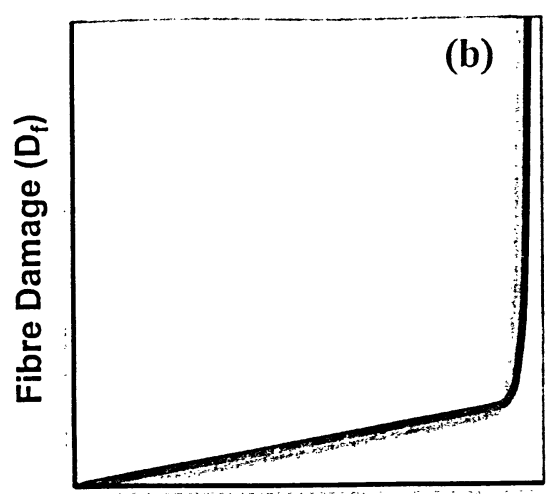

Life Fraction $\left(\mathbf{n} / \mathrm{N}_{\mathrm{f}}\right)$

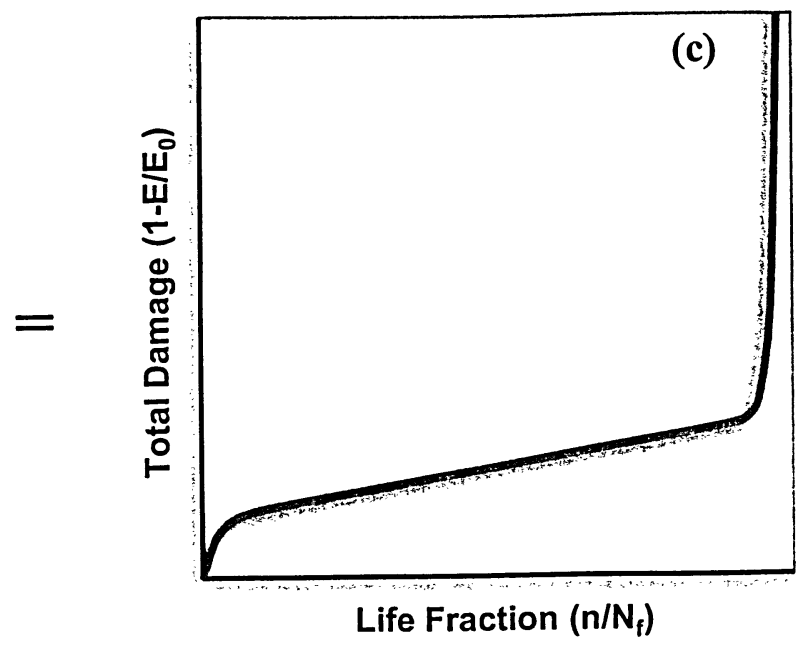

Fig. 4.6: A typical damage versus life fraction diagram of cortical bone under tensile fatigue loading presents (a) matrix and (b) fibres and (c) the overall fatigue damage. 


\subsubsection{Elements Affecting the Damage Assessment of Cortical Bone}

Euqtion (4.13) is developed to assess fatigue damage in cortical bone realistically as bone structure is subjected to cyclic stresses. The proposed damage model uniquely addresses various parameters affecting damage in cortical bone such as osteon volume fraction, mechanical properties of bone constituents, donor age, magnitude of applied cyclic stresses and the interfacial strength in cortical bone.

The following section will describe the elements affecting the damage assessment of cortical bone and develop their pertinent relationships in order to realistically assess fatigue damage of cortical bone using the proposed damage model (equation (4.13)).

\subsubsection{Volume Fraction of Osteons}

Volume fraction of osteons can basically be measured as the ratio of the total osteon area to the cross section area of the cortical bone. It has been shown [34] that age can be estimated from the percentage of non-remodelled cortical bone. Secondary osteon (fibres) are the direct result of remodelled bone. In fact, percentage of non-remodelled bone is the complement of osteon density in making the cortical bone. However, there are many factors affecting the number of osteons in bone specimens. Some of these factors are as follows:

- Regional variations have an effect on the quantity of osteons in each cross section of the bone. These regions are Anterior (front), Posterior (rear), Lateral, and Medial. This variable also affects the mechanical properties of bone structure and its microconstituents.

- Remodelling directly changes the quantity and density of the osteons in bone. This phenomenon has a substantial effect on the number of secondary osteons. The rate of remodelling can be influenced by physical activities, weight and bone disease.

- Age and gender also have a great effect on the number of osteons in bone specimens. Age has a detrimental effect on the strength of the bone. However, to comply with and react to different loading conditions of real life, cortical bone undergoes remodelling and changes its primary osteon type areas to secondary or Haversian system type. This phenomenon results in better crack arresting mechanism discussed in this thesis. 
Amongst the above mentioned effects on osteon volume fraction, age is the most influential biological parameter that has been quantitatively studied. Taylor and Lee [35] measured the percentage of primary bone for different donor ages. These data are presented in Fig. 4.7a. This figure shows the percent primary bone (non-remodelled bone) left in specimens from different human ages. Through mathematical evaluation and comparison to other test results it has been found that a function like "exp $(-0.04 \times$ Age $)$ " closely resembles the trend in the amount of primary bone drop, or in another word, increase in the amount of remodelled bone (secondary osteons) in terms of age.

Primary bone is replaced by secondary osteons through the remodelling process during the life of human being starting from zero secondary osteons at birth to an all remodelled secondary osteon type bone in older ages. Therefore, reduction in primary bone directly increases the quantity and density of the secondary osteons.

On the other hand, the process of remodelling constantly destroys and recreates new bone, resulting in some osteon fragments which cannot be considered as full osteons (fibres). Matrix in bone composite is considered to be the interstitial bone consisting of nonremodelled bone and osteon fragments separated from the full osteons by cement lines. The amount of osteon fragments in different age groups is not well known. To approximately account for osteon fragments as part of the matrix, the term "0.002xAge", which has a maximum value of 0.20 at the age of 100 , is added to the amount of non-remodelled bone. This term guarantees a minimum value of 0.20 for the matrix volume fraction. The term for osteon fragments is linearly reduced to zero at early years of life, as does the amount of secondary osteons. Therefore, matrix volume fraction can be calculated as:

$$
V_{m}=\exp (-0.04 \cdot A)+0.002 \cdot A
$$

where $A$ is donor age. Assuming bone is consisted of two phases: i) full osteons and ii) nonremodelled primary bone and osteon fragments, then the osteon volume fraction at any age can be calculated from the following function:

$$
V_{f}=1-V_{m}
$$

The proposed equation to calculate the osteon density (fibre volume fraction) is then given as: 


$$
V_{f}=1-[\exp (-0.04 \cdot A)+0.002 \cdot A]
$$

Fig. $4.7 \mathrm{~b}$ represents the variation of osteon volume fraction with donor age.
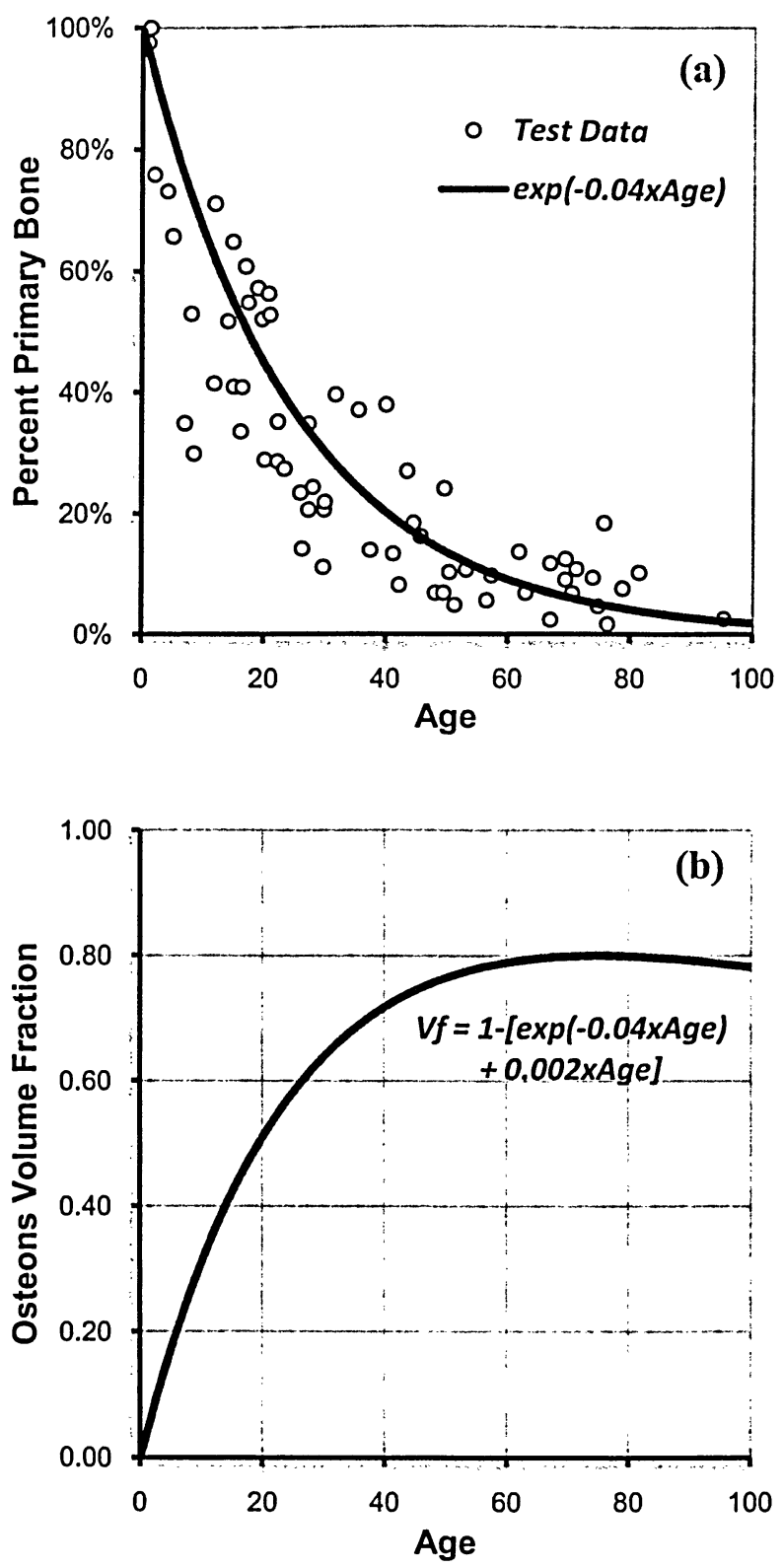

Fig. 4.7: Relationship between the percent primary bone and age. Data from Taylor and Lee [35] (shown with circles). a) Function exp(-0.04.Age) shows good agreement to test results. b) diagram of $V_{f}$ (osteon volume fraction) in terms of 'Age' equation (4.16). 
Equation (4.16) can also be used for age estimation by measuring the volume fraction of osteons using imaging instruments and techniques. In this equation it is assumed that at the very early ages there is no secondary osteons (Haversian system) type of bone. Fig. 4.8 schematically shows osteons and osteon fragments in cortical bone.

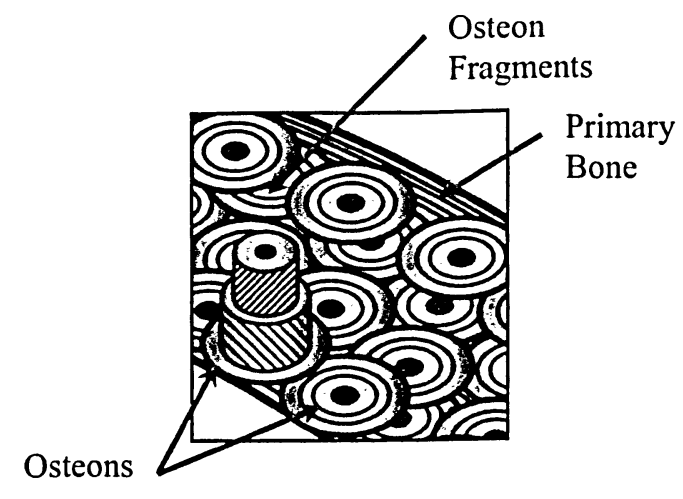

Fig. 4.8: Osteon fragments are old osteons partially replaced by new remodelled bone. Osteon fragments (as part of the interstitial bone) are considered the matrix in cortical bone.

There are factors other than age which affects the osteon density. Taking all these factors into account for the exact calculation of osteon volume fraction needs more research work and laboratory tests. To quantify the effect of other factors in the value of the osteon volume fraction an arbitrary coefficient of $\alpha$ is introduced into equation (4.16) as:

$$
V_{f}=\alpha \cdot\{1-[\exp (-0.04 \cdot A)+0.002 \cdot A]\}
$$

The coefficient, $\alpha$, has a value of unity for most bone test specimens.

\subsubsection{Secant Modulus of Osteons}

Modulus of elasticity of osteons $\left(E_{f}\right)$ can be measured by osteon push-out and monotonic tensile tests on a single osteon which is a delicate process. There have been some efforts to relate the stiffness of osteons to its constituents' mechanical and physical properties [12]. Most of the models take into account the effect of mineralization of collagen fibres and the arrangement type and orientation of these collagen fibres. Recent studies have shown that osteon structural differences may be related to collagen fibre orientation, and are likely to affect bone mechanical performance [36]. 
Katz [37] developed a model to calculate bone stiffness. This model which can also be used to calculate secant modulus of osteons is given as:

$$
E_{f}=\frac{E_{c o} V_{c o}\left(1-v_{f} v_{c o}\right)}{1-v_{c o}^{2}}+\sum E_{h a} V_{h a} \alpha_{n}\left(\cos ^{4} \varphi_{n}-v_{f} \cos ^{2} \varphi_{n} \sin ^{2} \varphi_{n}\right)
$$

where subscript $c o$ refers to collagen and subscript $h a$ refers to hydroxyapatite minerals, $v$ is the Poisson's ratio; $\alpha_{n}$ is the fraction of the apatite crystallites that line at an angle $\varphi_{n}$ with respect to loading axis. The first part of the equation refers to collagen, which is assumed to have the same modulus in all directions. The second part gives the effect of apatite minerals. Equation (4.18) can be used to estimate the value of $E_{f}$. However, values of $E_{f}$, used in this study, were extracted from sources of fatigue damage data for cortical bone samples [24], [25], [32], and [39].

\subsubsection{Applied Cyclic Stress versus Fatigue Life}

Applied cyclic stress has a direct impact on the fatigue behaviour of cortical bone. Cortical bone fatigue life can be related to the cyclic stress range by a power-law equation as [38]:

$$
N_{f}=0.5 \cdot\left(\frac{\Delta \sigma}{118.96}\right)^{-18.2}
$$

where $\Delta \sigma$ is the cyclic stress range in MPa. Constants and the exponent in equation (4.19) were obtained by Pattin et al. [24] conducting fatigue tests on human cortical bone under tensile loading.

In the proposed damage model, both fibre and matrix damage terms were defined as functions of cyclic stress magnitudes. Damage functions vary between zero to a maximum of unity. Thus, the effect of stress magnitudes is also incorporated to both damage functions of fibres (osteons) and matrix (interstitial bone) as a function of the normalized applied stress:

$$
r=100 \cdot \frac{\Delta \sigma}{E^{*}}
$$

where $E^{*}$ is the tangent modulus of cortical bone prior to fatigue test. Ratio $r$ also varies between zero to one. $\Delta \sigma / E^{*}$ is referred as the effective strain range in the cyclic loadings. 
Note that $E_{0}$ in equations (4.8) and (4.9) denotes secant modulus measured from the first loading cycle while $E^{*}$ value is the tangent modulus of the elastic region of stress-strain curve obtained under monotonic tensile test.

\subsubsection{Cement Line Interfacial Strength}

Interfacial strength between the osteons (fibres) and interstitial bone (matrix) is used as an influential parameter in the proposed fatigue damage model of cortical bone. Parameter $f$, the interfacial strength, in equations (4.13) is greatly dependent on the shear and deboning strength of the cement lines separating the osteons and the interstitial bone [36]. In this model, the parameter ' $f$ ' is defined as the ratio of the maximum debonding shear strength, $\tau_{d b}$, to the maximum interfacial shear strength of the cement lines, $\tau_{i}$ and varies between zero and unity:

$$
f=\frac{\tau_{d b}}{\tau_{i}}
$$

Table 4.1 represents maximum debonding shear strength, $\tau_{d b}$, and maximum interfacial shear strength, $\tau_{i}$, at various regions, osteon types, and failure modes.

Table 4.1: Shear Strengths of cortical bone cement lines [36].

\begin{tabular}{|c|c|c|c|c|}
\hline \multicolumn{2}{|r|}{ Subject } & $\begin{array}{l}\text { Maximum interfacial } \\
\text { shear strength, } \tau_{i}(\mathrm{MPa})^{*}\end{array}$ & $\begin{array}{c}\text { Maximum debonding } \\
\text { shear strength, } \tau_{d b}(\mathrm{MPa})^{*}\end{array}$ & $\begin{array}{l}\text { Ratio of } \\
f=\tau_{d b} / \tau_{i}\end{array}$ \\
\hline \multirow{4}{*}{ Region } & Dorsal & 86.3 & 40.5 & 0.47 \\
\hline & Lateral & 69.1 & 35.4 & 0.51 \\
\hline & Medial & 69.1 & 26.8 & 0.39 \\
\hline & Palmar & 68.0 & 24.0 & 0.35 \\
\hline \multirow{4}{*}{$\begin{array}{l}\text { Osteon } \\
\text { Type }\end{array}$} & Alternating & 76.3 & 22.8 & 0.30 \\
\hline & Bright field & 82.6 & 40.3 & 0.49 \\
\hline & Dark field & 63.6 & 32.1 & 0.50 \\
\hline & Hooped & 71.0 & 31.4 & 0.44 \\
\hline Failure & Cement line & 70.0 & 30.7 & 0.44 \\
\hline Mode & Interlamellar & 76.7 & 32.7 & 0.43 \\
\hline
\end{tabular}

* Values of $\tau_{d b}$ and $\tau_{i}$ have variations of \pm 7.7 .

The values of $f$ used in the proposed damage model have been determined by Bigley et al. [36] who conducted interfacial strength and osteon push-out tests on cortical bone samples. 


\section{Chapter 5}

\section{Evaluation of the Proposed Fatigue Damage Model}

In this chapter, the proposed fatigue damage model for human cortical bone is assessed and compared against the actual test results. The test data is from various human age groups and have been obtained under different magnitudes of cyclic stresses. The test data were extracted from the literature and used to evaluate fatigue damage $\left(1-E / E_{0}\right)$ as the number of cycles progressed. Test data presented in the this chapter have been performed in four different laboratories and are described in detail.

\subsection{Evaluation Procedure}

A computer program was developed to analyze fatigue damage of cortical bone as number of cycles increased through several steps:

i. Determine mechanical properties of cortical bone $\left(E_{0}, V_{f}, E_{f}, \Delta \sigma\right.$, and Age).

ii. Calculate cyclic loading variables of, $r$ and $f$.

iii. Calculate number of cycles to failure, $N_{f}$.

iv. Compute damage components for osteons and interstitial bone as the number of cycles progresses.

The flowchart in Fig. 5.1 presents the steps in the evaluation of the proposed fatigue damage model. Test methods and procedures for each test data source are briefly explained. 


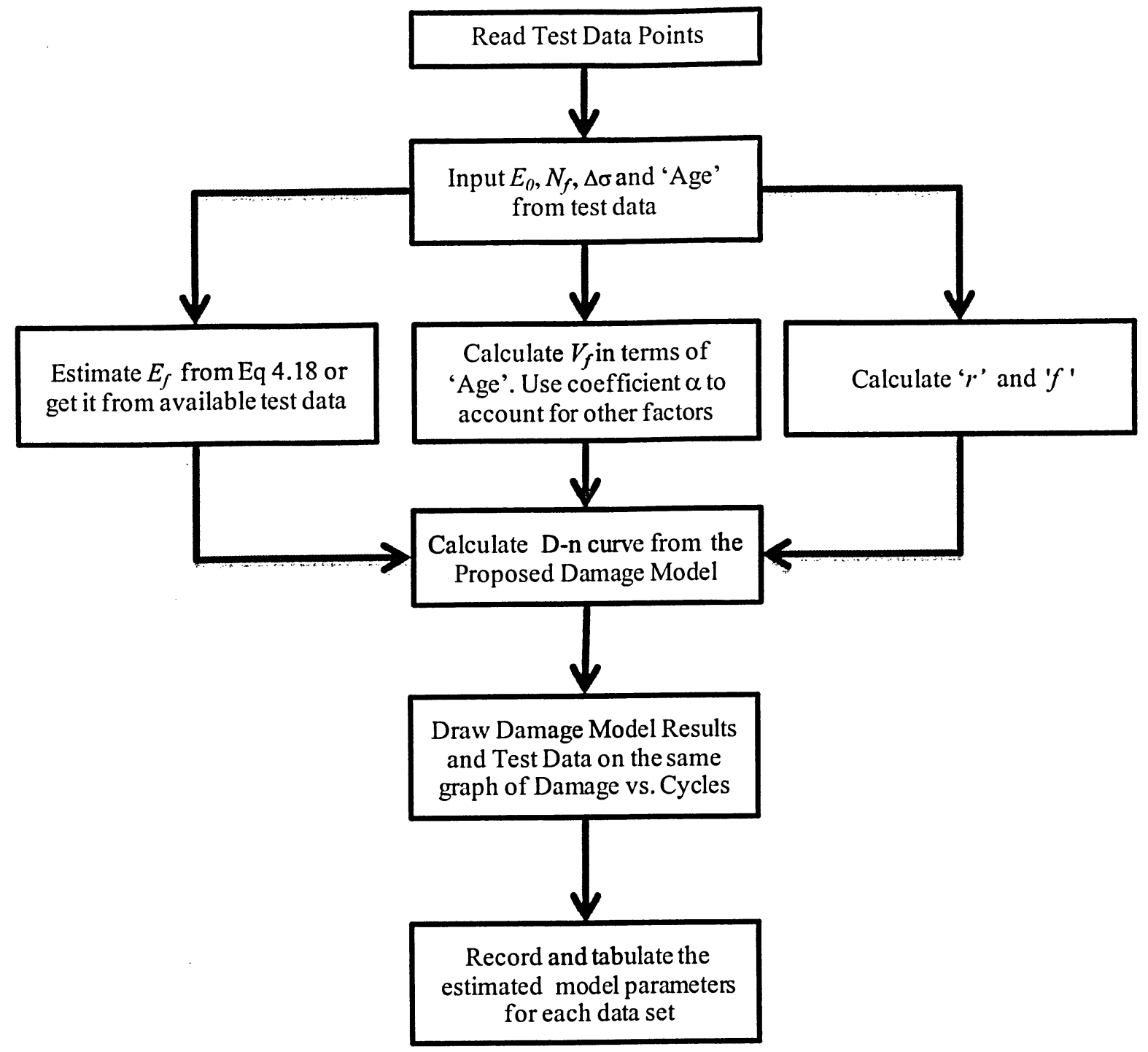

Fig. 5.1: Process chart for the evaluation of the proposed fatigue damage model against test data. 


\subsection{Test Data of Pattin et al.}

Pattin et al. [24] machined and tested several specimens from femoral mid-shafts of male cadavers (ages 18-49) to study the fatigue damage in human cortical bone based on the mechanical property degradation. Cylinders of $4 \mathrm{~mm}$ diameter and 25-30 $\mathrm{mm}$ in length oriented along the long axis of the bone were prepared. The central $8 \mathrm{~mm}$ of these specimens were then turned down to $3 \mathrm{~mm}$ on a lathe. Specimens were kept frozen in wet paper towels in plastic bags at $-20^{\circ} \mathrm{C}$ except during testing and machining. The specimens were then tested using a MTS 810 servo-hydraulic testing machine. Specimens were maintained at $37^{\circ} \mathrm{C}$ and kept wet throughout testing. The loading waveform was a $2 \mathrm{~Hz}$ haversine for the tensile fatigue tests under load-controlled fatigue to fracture at a single stress level.

Table 5.1 lists the mechanical properties of four sample test specimens along with the input parameters used in the proposed fatigue damage model to assess the fatigue damage of cortical bone samples. Values of $r, V_{f}, E_{f}^{*}$ are listed this table. Figures 5.2 and 5.3 compare experimental D-n data reported by Pattin et al. [24] with D-n curves predicted based on the proposed damage model.

Table 5.1: Test data and parameters of Pattin et al [24].

\begin{tabular}{|c|c|c|c|c|c|c|c|c|c|c|}
\hline \multirow{3}{*}{$\begin{array}{c}\text { Data } \\
\#\end{array}$} & \multicolumn{4}{|c|}{ Test Specimen Parameters } & \multirow{2}{*}{\multicolumn{3}{|c|}{ Input Parameters }} & \multirow{2}{*}{\multicolumn{3}{|c|}{ Calculated Parameters }} \\
\hline & \multirow{2}{*}{$\begin{array}{c}\text { Age } \\
\text { (years) }\end{array}$} & \multirow{2}{*}{$\underset{(M P a)}{\Delta \sigma}$} & \multirow{2}{*}{$\begin{array}{c}\boldsymbol{E}_{0} \\
(\mathrm{MPa})\end{array}$} & & & & & & & \\
\hline & & & & & $\overline{E_{f}(M P a)}$ & $\alpha$ & $f$ & $\boldsymbol{r}$ & $V_{f}$ & $E_{f}^{*}$ \\
\hline 1 & 39 & 83 & 18.04 & 417 & 9.02 & 0.60 & 0.40 & 0.408 & 0.47 & 0.12 \\
\hline 2 & 21 & 82.3 & 19.261 & 7688 & 14.45 & 1.00 & 0.22 & 0.355 & 0.56 & 0.28 \\
\hline 3 & 18 & 70.7 & 19.98 & 18658 & 19.98 & 1.10 & 0.17 & 0.331 & 0.56 & 0.54 \\
\hline 4 & 39 & 55.0 & 20.62 & 16331 & 20.62 & 1.05 & 0.0 & 0.267 & 0.82 & 0.82 \\
\hline
\end{tabular}

Figures 5.2 and Fig. 5.3 show how closely the proposed damage model can predict the fatigue damage of cortical bone samples at various cyclic stress magnitudes. 


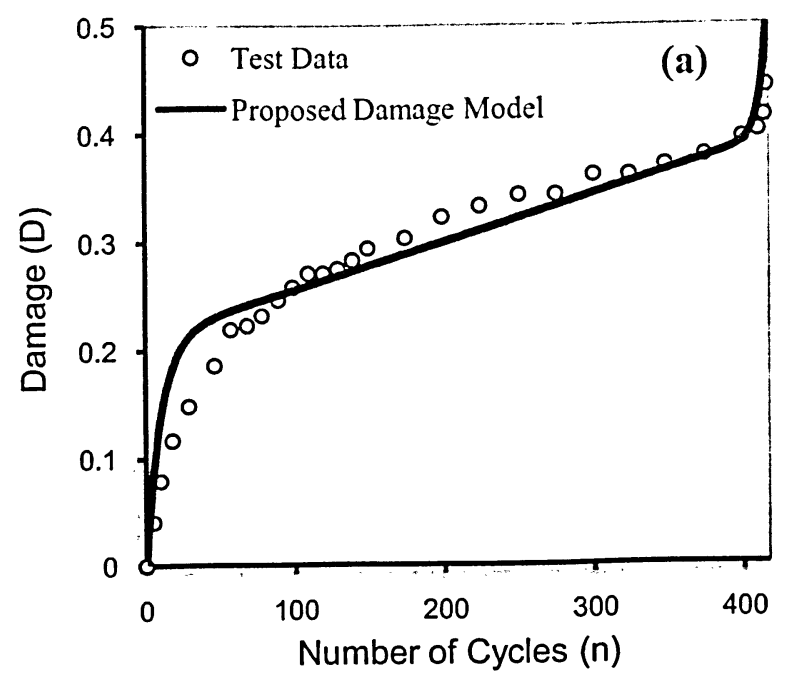

\begin{tabular}{|l|r|}
\hline$\triangle \sigma(M P a)$ & 83.00 \\
\hline$E_{0}(G P a)$ & 18.04 \\
\hline$N_{f}$ & 417 \\
\hline Age & 39 \\
\hline \hline$E_{f}(G P a)$ & 9.02 \\
\hline$V_{f}$ & 0.47 \\
\hline$f$ & 0.4 \\
\hline
\end{tabular}

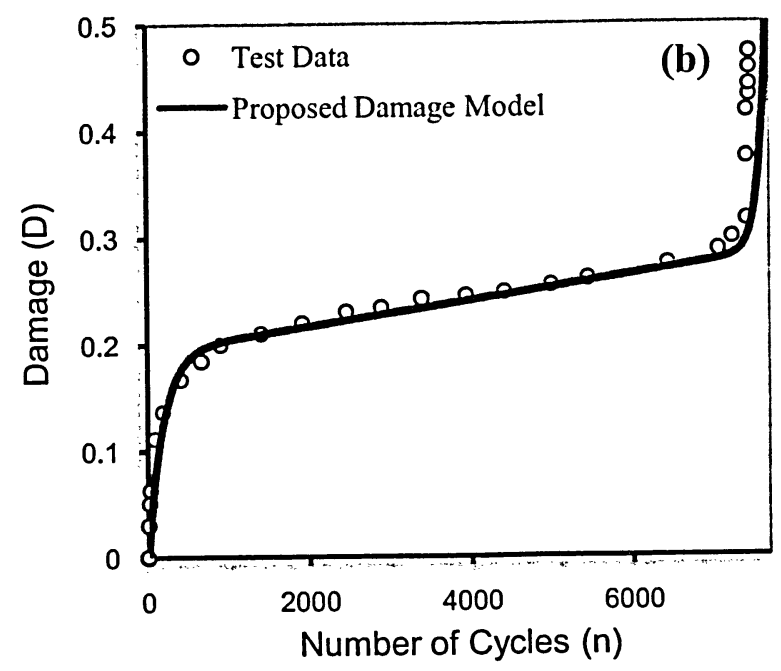

\begin{tabular}{|l|r|}
\hline$\triangle \sigma(M P a)$ & 82.30 \\
\hline$E_{o}(G P a)$ & 19.26 \\
\hline$N_{f}$ & 7688 \\
\hline$A g e$ & 21 \\
\hline \hline$E_{f}(G P a)$ & 14.45 \\
\hline$V_{f}$ & 0.56 \\
\hline$f$ & 0.22 \\
\hline
\end{tabular}

Fig. 5.2: Pattin et al. [24] test data compared with predicted damage based on the proposed fatigue damage model, Eq. (4.13). 


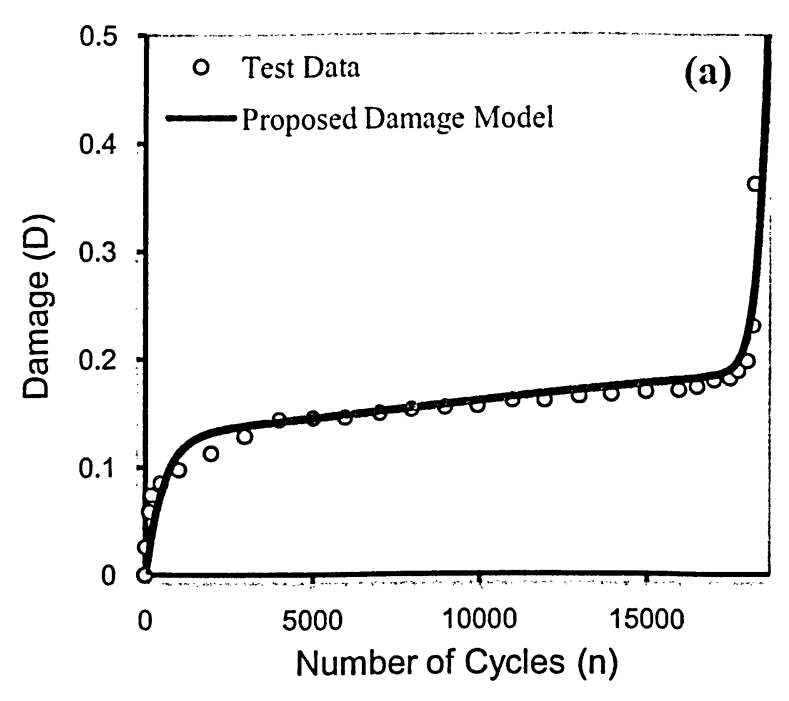

\begin{tabular}{|l|r|}
\hline$\Delta \sigma(M P a)$ & 70.70 \\
\hline$E_{o}(G P a)$ & 19.98 \\
\hline$N_{f}$ & 18658 \\
\hline Age & 18 \\
\hline \hline$E_{f}(G P a)$ & 19.98 \\
\hline$V_{f}$ & 0.56 \\
\hline$f$ & 0.17 \\
\hline
\end{tabular}

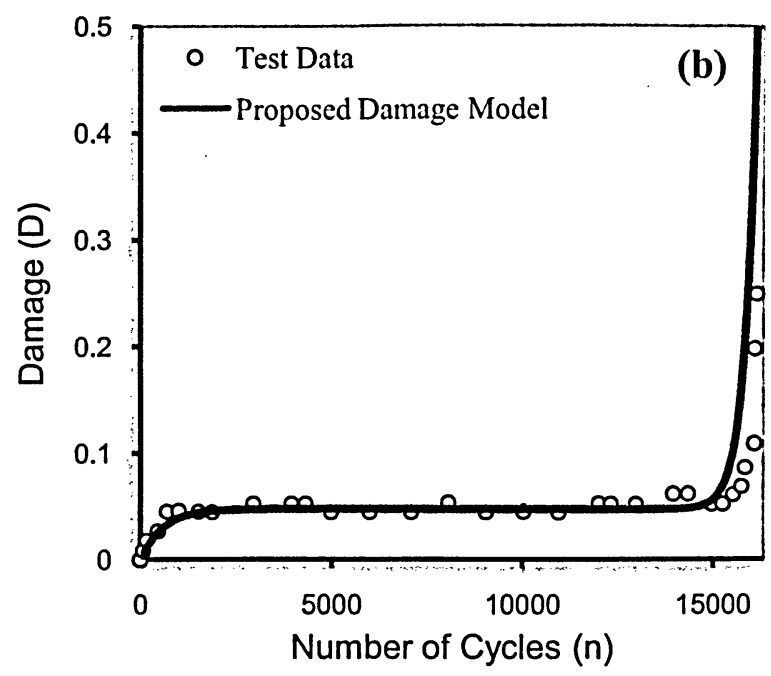

\begin{tabular}{|l|r|}
\hline$\Delta \sigma(M P a)$ & 55.00 \\
\hline$E_{o}(G P a)$ & 20.62 \\
\hline$N_{f}$ & 16331 \\
\hline$A g e$ & 39 \\
\hline \hline$E_{f}(G P a)$ & 20.62 \\
\hline$V_{f}$ & 0.82 \\
\hline$f$ & 0 \\
\hline
\end{tabular}

Fig. 5.3: Pattin et al. [24] test data compared with predicted damage based on the proposed fatigue damage model, Eq. (4.13). 


\subsection{Test Data by Griffin et al.}

In the tests performed by Griffin et al. [32], beams with nominal dimensions of $4 \times 10 \times 100 \mathrm{~mm}$ were machined from the mid-diaphysis of six human femurs obtained from unembalmed adult cadavers and stored frozen at $-4^{\circ} \mathrm{C}$. The means and standard deviations of their porosity, initial elastic modulus, and basic fuchsin-stained microcrack density were $6.9 \pm 1.5 \%$. $15.9 \pm 1.4 \mathrm{GPa}$ and $2.37 \pm 1.95 / \mathrm{mm}^{2}$, respectively. The beams were tested to fatigue failure in four-point bending in accordance with American Society for Testing and Materials Standard. The tests were done in load-control with the specimens submerged in saline solution at $37^{\circ} \mathrm{C}$ and the periosteal side of the beam in tension.

Tests were performed with peak strains of 6000,6500 , and 7500 micro-strain. In all cases, the low end of the load range was $10 \mathrm{~N}$. Specimens from the medial, lateral and anterior cortices were randomly distributed among the experimental groups.

Table 5.2 presents three sets of test results extracted from the work of Griffin et al. [32]. Age, cyclic stress range, secant modulus and fatigue life values are listed for each test. Calculated values of $r, V_{f}, E_{f}^{*}$ are also listed. The results of the proposed damage model are compared with the fatigue test data of cortical bone in a 50 -year-old female tested under various cyclic stresses. The predicted and experimental data are presented in Fig. 5.4. The predicted D-n curve is in good agreement with experimental data presented in this figure.

Table 5.2: Test data and parameters from Griffin et al. [32]

\begin{tabular}{|c|c|c|c|c|c|c|c|c|c|c|}
\hline \multirow{3}{*}{$\begin{array}{c}\text { Data } \\
\#\end{array}$} & \multicolumn{4}{|c|}{ Test Specimen Parameters } & \multirow{2}{*}{\multicolumn{3}{|c|}{ Input Parameters }} & \multirow{2}{*}{\multicolumn{3}{|c|}{ Calculated Parameters }} \\
\hline & \multirow{2}{*}{$\begin{array}{c}\text { Age } \\
\text { (years) }\end{array}$} & \multirow{2}{*}{$\begin{array}{c}\Delta \sigma \\
(M P a)\end{array}$} & \multirow{2}{*}{$\begin{array}{c}E_{0} \\
(\mathrm{MPa})\end{array}$} & \multirow{2}{*}{$N_{f}$} & & & & & & \\
\hline & & & & & $E_{f}(M P a)$ & $\alpha$ & $f$ & $\boldsymbol{r}$ & $V_{f}$ & $E_{f}^{*}$ \\
\hline 1 & 50 & 69.2 & 15.9 & 21500 & 17.49 & 1.00 & 0.25 & 0.435 & 0.85 & 0.94 \\
\hline 2 & 50 & 73 & 15.9 & 6150 & 16.70 & 1.00 & 0.25 & 0.459 & 0.85 & 0.90 \\
\hline 3 & 50 & 80.9 & 15.9 & 900 & 15.90 & 1.00 & 0.55 & 0.509 & 0.85 & 0.85 \\
\hline
\end{tabular}




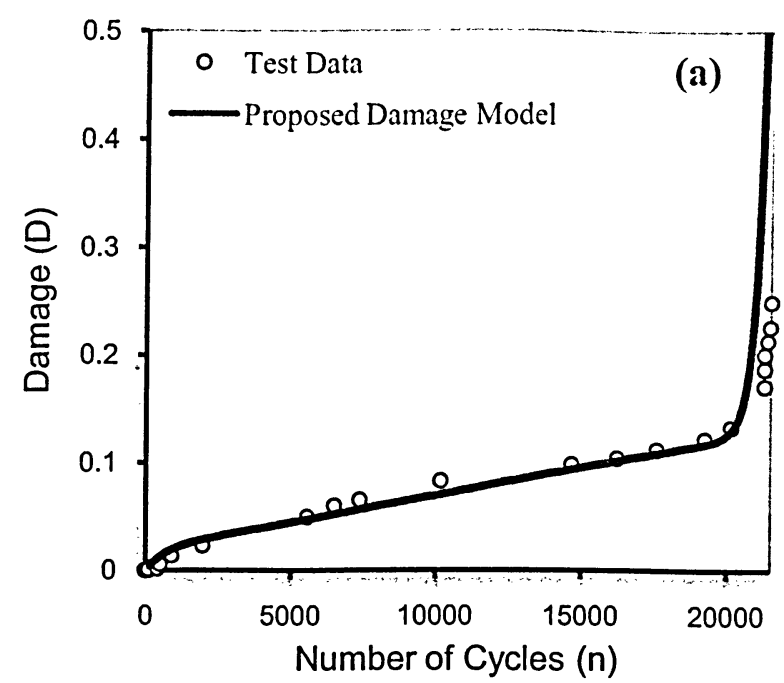

\begin{tabular}{|l|r|}
\hline$\Delta \sigma(M P a)$ & 69.20 \\
\hline$E_{o}(G P a)$ & 15.90 \\
\hline$N_{f}$ & 21500 \\
\hline$A g e$ & 50 \\
\hline \hline$E_{f}(G P a)$ & 17.90 \\
\hline$V_{f}$ & 0.85 \\
\hline$f$ & 0.25 \\
\hline
\end{tabular}

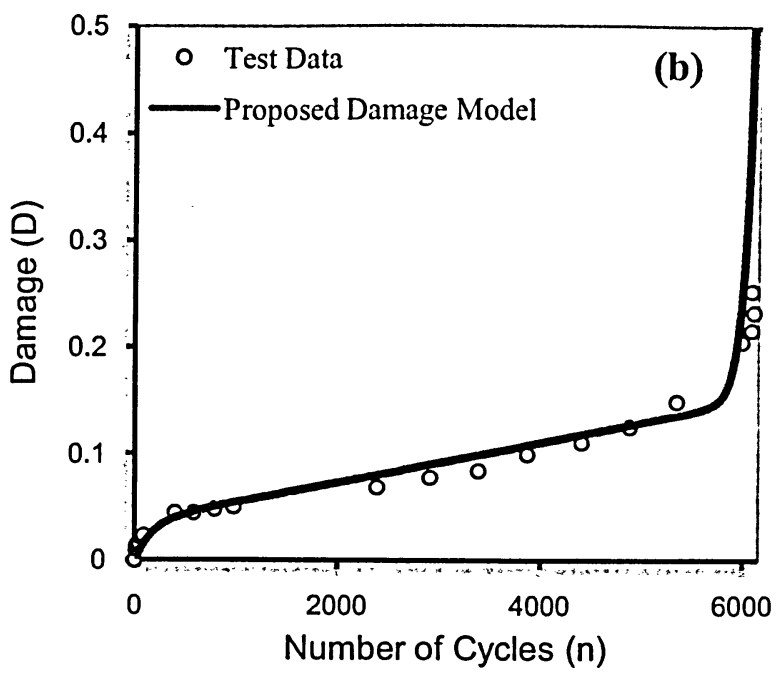

\begin{tabular}{|l|r|}
\hline$\triangle \sigma(M P a)$ & 73.50 \\
\hline$E_{0}(G P a)$ & 15.90 \\
\hline$N_{f}$ & 6150 \\
\hline$A g e$ & 50 \\
\hline \hline$E_{f}(G P a)$ & 16.70 \\
\hline$V_{f}$ & 0.85 \\
\hline$f$ & 0.25 \\
\hline
\end{tabular}

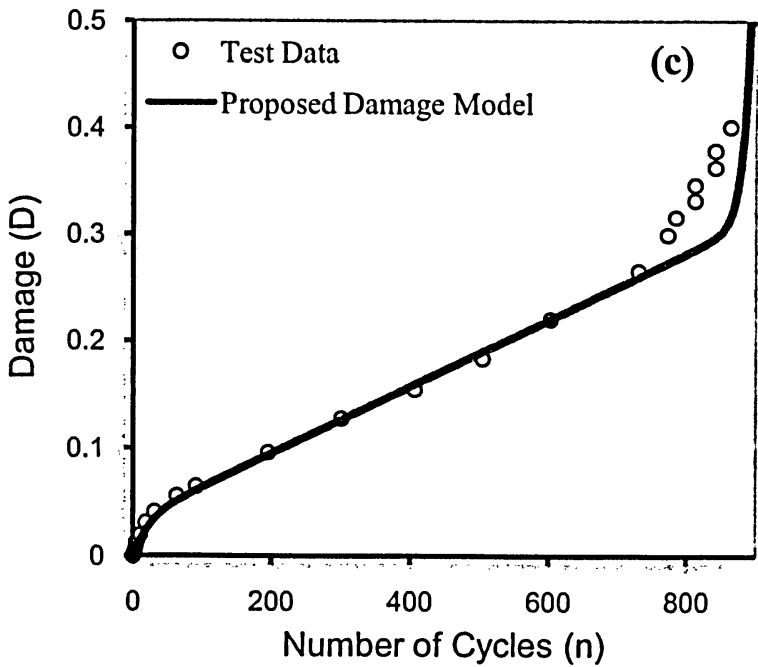

\begin{tabular}{|l|r|}
\hline$\triangle \sigma(\mathrm{MPa})$ & 80.90 \\
\hline$E_{0}(\mathrm{GPa})$ & 15.90 \\
\hline$N_{f}$ & 900 \\
\hline Age & 50 \\
\hline \hline$E_{f}(\mathrm{GPa})$ & 15.90 \\
\hline$V_{f}$ & 0.85 \\
\hline$f$ & 0.55 \\
\hline
\end{tabular}

Fig. 5.4: Griffin et al. [32] test data compared with predicted damage based on the proposed fatigue damage model, Eq. (4.13). 


\subsection{Test Data by Zioupos et al.}

Zioupos and Casinos [25] investigated the accumulation of fatigue microdamage in human cortical bone of two different ages. Specimens came from the mid-distal part of the diaphyses of two female human femurs obtained during routine autopsies. Twelve samples were cut from the femur of a 27-year-old female (height $1.60 \mathrm{~m}$. weight $69.5 \mathrm{~kg}$ ) and eight samples from a 56-year-old female (height $1.65 \mathrm{~m}$, weight $65 \mathrm{~kg}$,). All specimens were cut on a longitudinal-tangential plane of the bones. The modulus of elasticity of the specimens were measured in a water bath at room temperature by 3-point bending in a screw-driven INSTRON 1122 materials testing machine. Afterwards the samples were turned to a dumbbell shape having a $4 \mathrm{~mm} \times 2 \mathrm{~mm}$ uniform cross-section over an approximately $15 \mathrm{~mm}$ long central region, with shoulders of $15 \mathrm{~mm}$ radius. During the whole preparation the samples were immersed in water at room temperature. For short intervals (minutes) between tests the specimens were kept in full strength Ringer's solution in the refrigerator at $4^{\circ} \mathrm{C}$, while for longer time intervals (hours) they were kept wrapped in water soaked cloth in airtight containers at $-20^{\circ} \mathrm{C}$.

Mechanical properties of the test specimens are listed in Table 5.3. The input and calculated values for various cyclic stress magnitudes are listed in this table.

Table 5.3: Test data and parameters from Zioupos and Casinos [25].

\begin{tabular}{|c|c|c|c|c|c|c|c|c|c|c|}
\hline \multirow{3}{*}{$\begin{array}{c}\text { Data } \\
\#\end{array}$} & \multicolumn{4}{|c|}{ Test Specimen Parameters } & \multirow{2}{*}{\multicolumn{3}{|c|}{ Input Parameters }} & \multirow{2}{*}{\multicolumn{3}{|c|}{ Calculated Parameters }} \\
\hline & \multirow{2}{*}{$\begin{array}{c}\text { Age } \\
\text { (years) }\end{array}$} & \multirow{2}{*}{$\begin{array}{c}\Delta \boldsymbol{\sigma} \\
(M P a)\end{array}$} & \multirow{2}{*}{$\begin{array}{c}E_{0} \\
(M P a)\end{array}$} & \multirow{2}{*}{$N_{f}$} & & & & & & \\
\hline & & & & & $E_{f}(M P a)$ & $\alpha$ & $f$ & $r$ & $V_{f}$ & $E_{f}^{*}$ \\
\hline 1 & 56 & 70 & 11.1 & 8053 & 12.43 & 1.00 & 0.25 & 0.631 & 0.88 & 0.99 \\
\hline 2 & 56 & 72.8 & 13.2 & 5677 & 13.86 & 1.00 & 0.25 & 0.552 & 0.88 & 0.93 \\
\hline 3 & 56 & 78.4 & 13.5 & 942 & 14.18 & 1.00 & 0.40 & 0.581 & 0.88 & 0.93 \\
\hline 4 & 56 & 83 & 13.9 & 124 & 13.90 & 1.00 & 0.40 & 0.597 & 0.88 & 0.88 \\
\hline 5 & 56 & 86.3 & 12.2 & 339 & 13.42 & 1.00 & 0.28 & 0.707 & 0.88 & 0.97 \\
\hline 6 & 56 & 93.1 & 13.7 & 17 & 14.66 & 1.00 & 0.35 & 0.680 & 0.88 & 0.94 \\
\hline 7 & 27 & 84.6 & 14.9 & 209480 & 19.07 & 1.15 & 0.10 & 0.568 & 0.75 & 0.96 \\
\hline 8 & 27 & 87.5 & 14.9 & 127590 & 18.63 & 1.15 & 0.15 & 0.587 & 0.75 & 0.94 \\
\hline 9 & 27 & 91.4 & 16.7 & 11863 & 20.88 & 1.15 & 0.25 & 0.547 & 0.75 & 0.94 \\
\hline 10 & 27 & 100.7 & 17.3 & 402 & 22.14 & 1.15 & 0.30 & 0.582 & 0.75 & 0.96 \\
\hline 11 & 27 & 104.8 & 15.1 & 392 & 19.63 & 1.15 & 0.50 & 0.694 & 0.75 & 0.98 \\
\hline
\end{tabular}


Figures 5.5-5.8 compare the predicted D-n curves for various cyclic stress magnitudes with the corresponding experimental data conducted by Zioupos and Casinos [25]. Comparison of experimental data and predicted curves show how successfully the proposed damage model assesses damage in cortical bone.

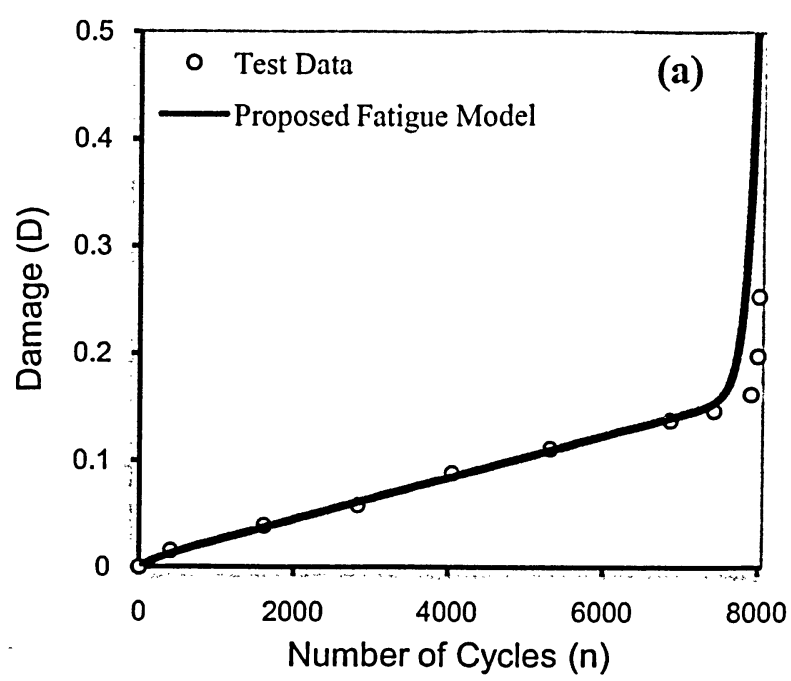

\begin{tabular}{|l|r|}
\hline$\Delta \sigma(M P a)$ & 70.00 \\
\hline$E_{0}(G P a)$ & 11.10 \\
\hline$N_{f}$ & 8053 \\
\hline Age & 56 \\
\hline \hline$E_{f}(G P a)$ & 12.43 \\
\hline$V_{f}$ & 0.88 \\
\hline$f$ & 0.25 \\
\hline
\end{tabular}

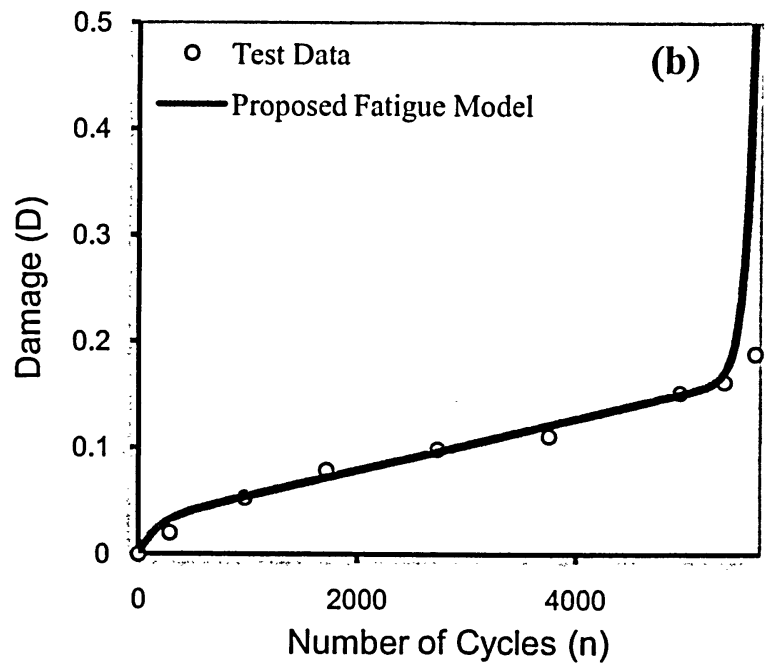

\begin{tabular}{|l|r|}
\hline$\triangle \sigma(M P a)$ & 72.80 \\
\hline$E_{0}(G P a)$ & 13.20 \\
\hline$N_{f}$ & 5677 \\
\hline Age & 56 \\
\hline \hline$E_{f}(G P a)$ & 13.86 \\
\hline$V_{f}$ & 0.88 \\
\hline$f$ & 0.25 \\
\hline
\end{tabular}

Fig. 5.5: Zioupos and Casinos [25] test data compared with predicted damage based on the proposed fatigue damage model, Eq. (4.13). 


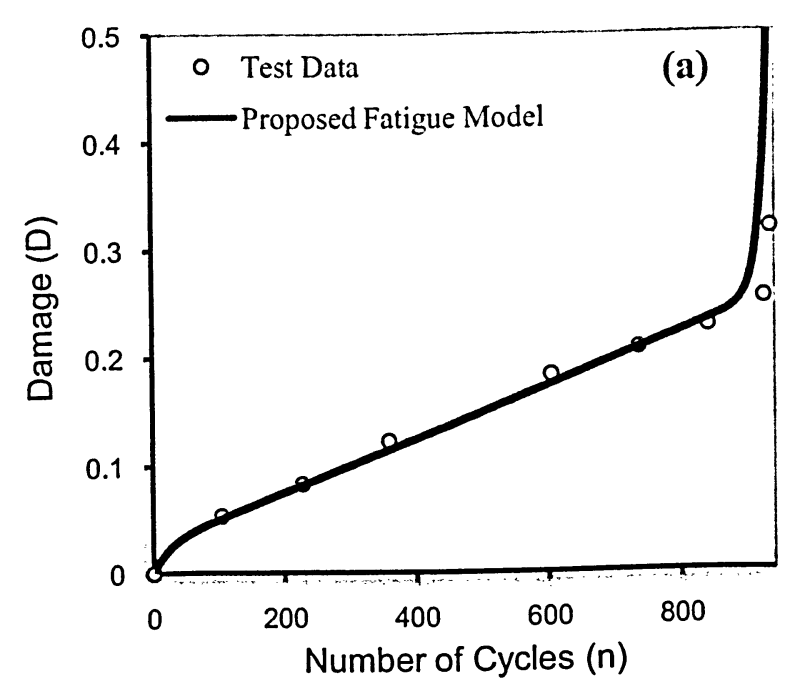

\begin{tabular}{|l|r|}
\hline$\triangle \sigma(M P a)$ & 78.40 \\
\hline$E_{o}(G P a)$ & 13.50 \\
\hline$N_{f}$ & 942 \\
\hline Age & 56 \\
\hline \hline$E_{f}(G P a)$ & 14.18 \\
\hline$V_{f}$ & 0.88 \\
\hline$f$ & 0.4 \\
\hline
\end{tabular}

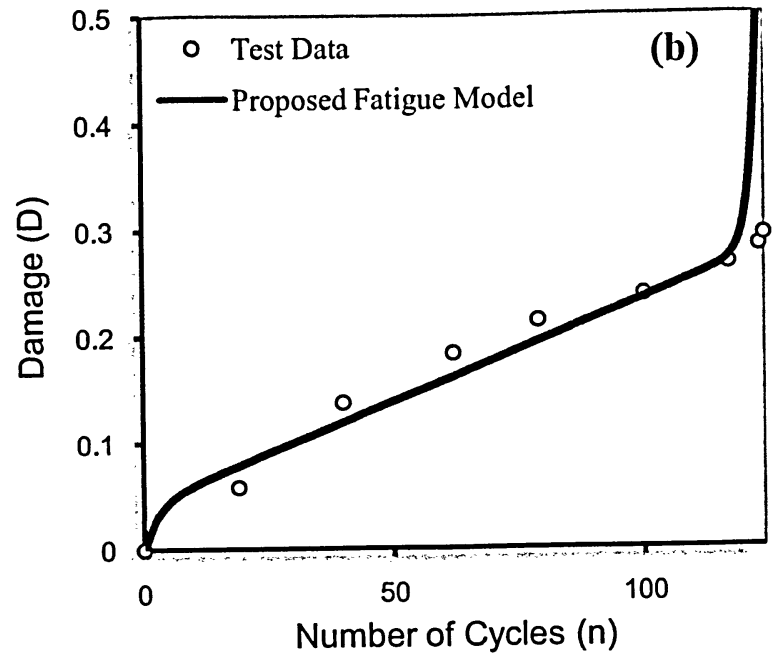

\begin{tabular}{|l|r|}
\hline$\Delta \sigma(M P a)$ & 83.00 \\
\hline$E_{o}(G P a)$ & 13.90 \\
\hline$N_{f}$ & 124 \\
\hline$A g e$ & 56 \\
\hline \hline$E_{f}(G P a)$ & 13.90 \\
\hline$V_{f}$ & 0.88 \\
\hline$f$ & 0.4 \\
\hline
\end{tabular}

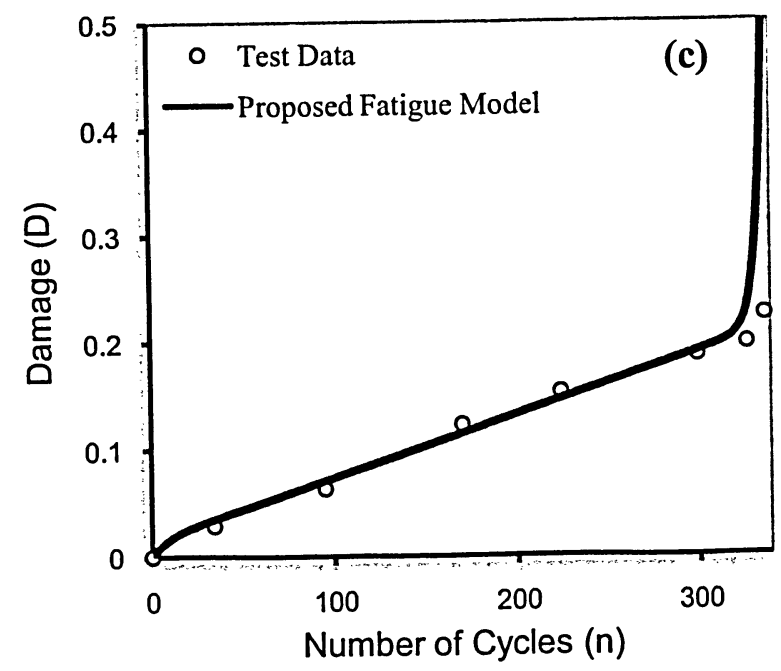

\begin{tabular}{|l|r|}
\hline$\Delta \sigma(M P a)$ & 86.30 \\
\hline$E_{0}(G P a)$ & 12.20 \\
\hline$N_{f}$ & 339 \\
\hline$A g e$ & 56 \\
\hline \hline$E_{f}(G P a)$ & 13.42 \\
\hline$V_{f}$ & 0.88 \\
\hline$f$ & 0.28 \\
\hline
\end{tabular}

Fig. 5.6: Zioupos and Casinos [25] test data compared with predicted damage based on the proposed fatigue damage model, Eq. (4.13). 


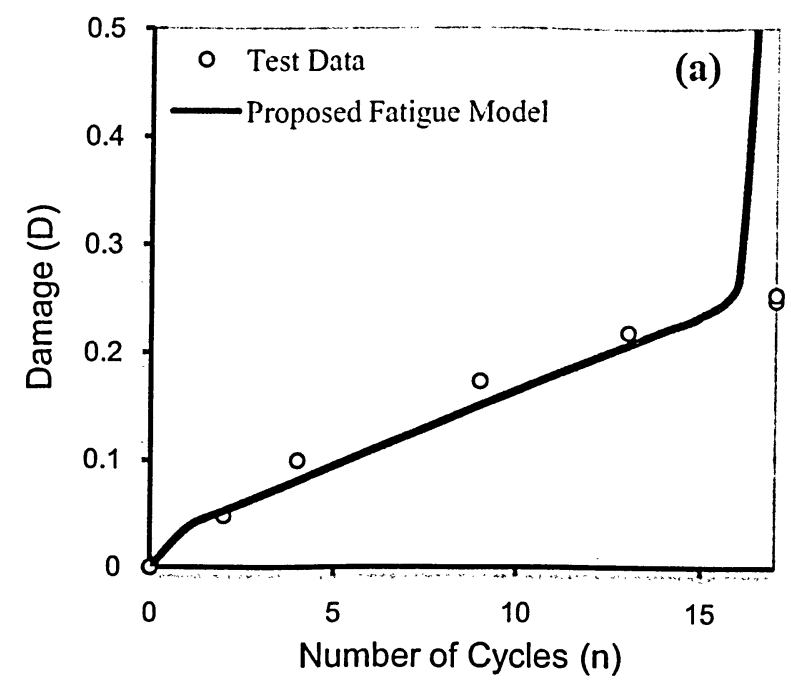

\begin{tabular}{|l|r|}
\hline$\triangle \sigma(M P a)$ & 93.10 \\
\hline$E_{0}(G P a)$ & 13.70 \\
\hline$N_{f}$ & 17 \\
\hline Age & 56 \\
\hline \hline$E_{f}(G P a)$ & 14.66 \\
\hline$V_{f}$ & 0.88 \\
\hline$f$ & 0.35 \\
\hline
\end{tabular}

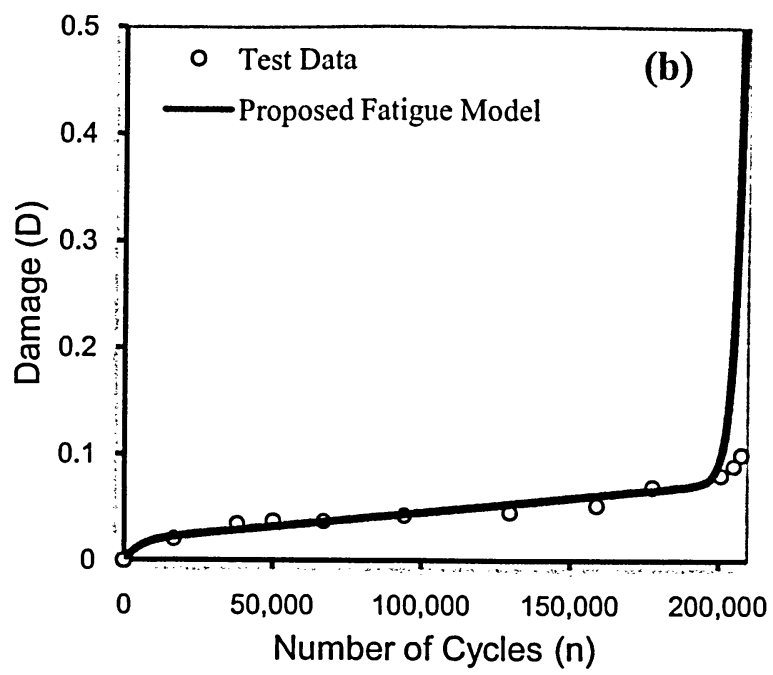

\begin{tabular}{|l|r|}
\hline$\triangle \sigma(M P a)$ & 84.60 \\
\hline$E_{0}(G P a)$ & 14.90 \\
\hline$N_{f}$ & 209480 \\
\hline Age & 27 \\
\hline \hline$E_{f}(G P a)$ & 19.07 \\
\hline$V_{f}$ & 0.75 \\
\hline$f$ & 0.1 \\
\hline
\end{tabular}

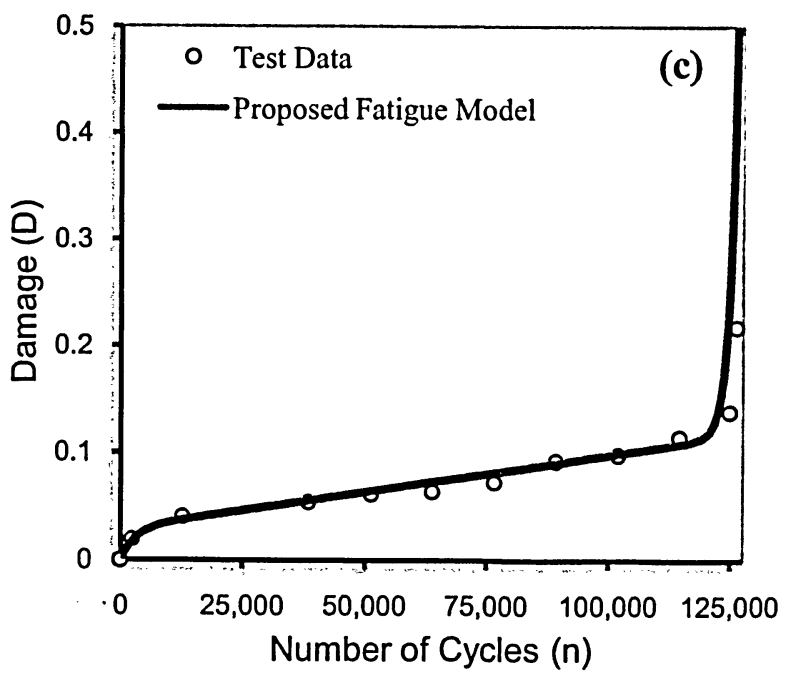

\begin{tabular}{|l|r|}
\hline$\triangle \sigma(\mathrm{MPa})$ & 87.50 \\
\hline$E_{0}(\mathrm{GPa})$ & 14.90 \\
\hline$N_{f}$ & 127590 \\
\hline Age & 27 \\
\hline \hline$E_{f}(\mathrm{GPa})$ & 18.63 \\
\hline$V_{f}$ & 0.75 \\
\hline$f$ & 0.15 \\
\hline
\end{tabular}

Fig. 5.7: Zioupos and Casinos [25] test data compared with predicted damage based on the proposed fatigue damage model, Eq. (4.13). 


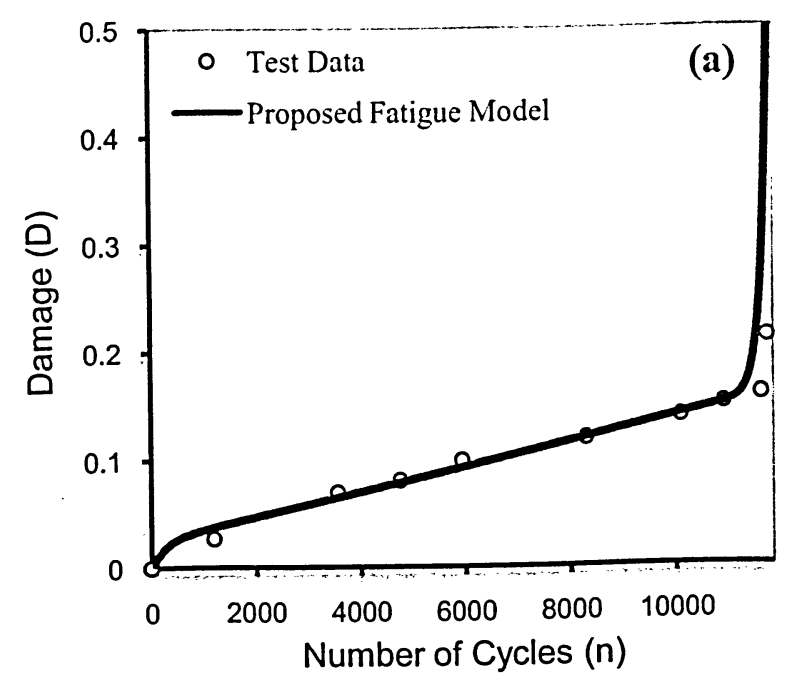

\begin{tabular}{|l|r|}
\hline$\triangle \sigma(M P a)$ & 91.40 \\
\hline$E_{o}(G P a)$ & 16.70 \\
\hline$N_{f}$ & 11863 \\
\hline$A g e$ & 27 \\
\hline \hline$E_{f}(G P a)$ & 20.88 \\
\hline$V_{f}$ & 0.75 \\
\hline$f$ & 0.25 \\
\hline
\end{tabular}

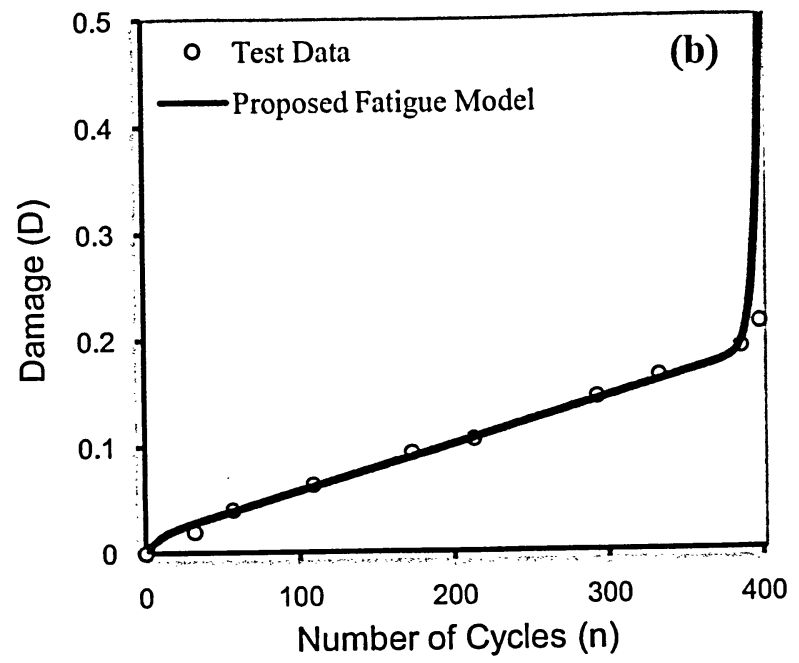

\begin{tabular}{|l|r|}
\hline$\triangle \sigma(\mathrm{MPa})$ & 100.70 \\
\hline$E_{0}(\mathrm{GPa})$ & 17.30 \\
\hline$N_{f}$ & 402 \\
\hline Age & 27 \\
\hline \hline$E_{f}(\mathrm{GPa})$ & 22.14 \\
\hline$V_{f}$ & 0.75 \\
\hline$f$ & 0.3 \\
\hline
\end{tabular}

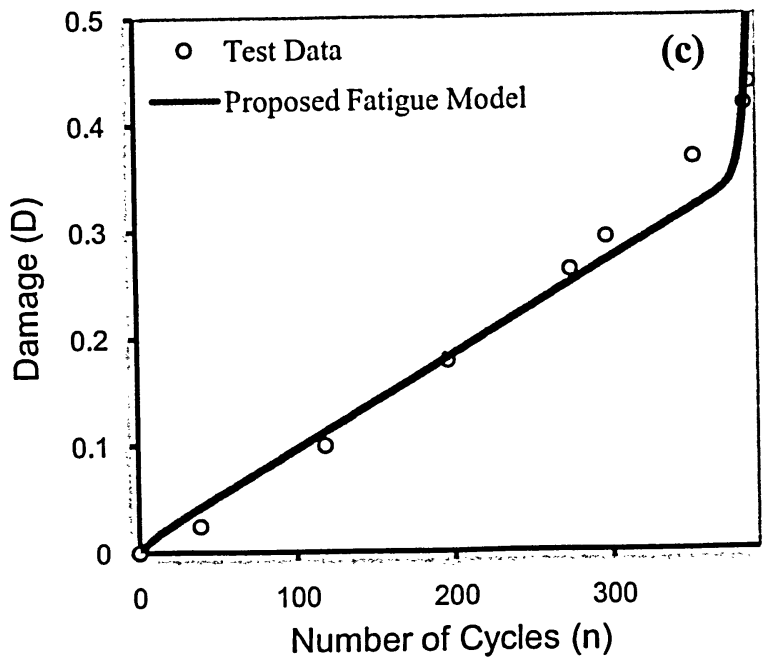

\begin{tabular}{|l|r|}
\hline$\triangle \sigma(M P a)$ & 104.80 \\
\hline$E_{o}(G P a)$ & 15.10 \\
\hline$N_{f}$ & 392 \\
\hline Age & 27 \\
\hline \hline$E_{f}(G P a)$ & 19.63 \\
\hline$V_{f}$ & 0.75 \\
\hline$f$ & 0.5 \\
\hline
\end{tabular}

Fig. 5.8: Zioupos and Casinos [25] test data compared with predicted damage based on the proposed fatigue damage model, Eq. (4.13). 


\subsubsection{Effect of Cyclic Stress Magnitude}

Equation (4.13) has a linear part which accounts for the stage II of the D-n curve. Slope of stage II is of great significance in studying fatigue damage mechanisms. This slope varies with the magnitude of the applied cyclic stress. Fig. 5.9 presents linear part of $D-n / N_{f}$ curve $\left(n / N_{f} \cong 0.15\right.$ to 0.9$)$ of Zioupos and Casinos [25] fatigue test data for 27-year-old and 56year-old bone specimens. As the applied cyclic stress magnitude increases the slope of the linear portion (stage II) also increases. This slope and its dependence on the applied cyclic stress have been taken into account in the proposed damage model (equation (4.13)) by introducing stage II damage as $r \cdot f \cdot \frac{n}{N_{f}}$, where $r$ is normalized cyclic stress multiplied by $f$ the shear strength along the cement line interface.

Fig. 5.10 presents the diagrams of slope $m$ versus stress magnitude for a set of bone specimens tested for ages 27 and 56. Fig. 5.10a shows a sharper trend of variation of $m$ with stress magnitude for age 27 while this trend is less steep for age of 56 shown in Fig. 5.10b. This may verify the impact of age and stress on the rate of damage in stage II of D-n/ $N_{f}$ diagrams.

Presumably with higher donor ages the cement line interfacial strength degrades which results in more moderate variation of the slope of fatigue curve in stage II with cyclic stress magnitudes. The regression line of data points for 56-year-old samples (c) presents a more moderate change of the slope $m$ with stress magnitude. Fig. 5.10c shows the drop in slope $m$ as donor age increases. 

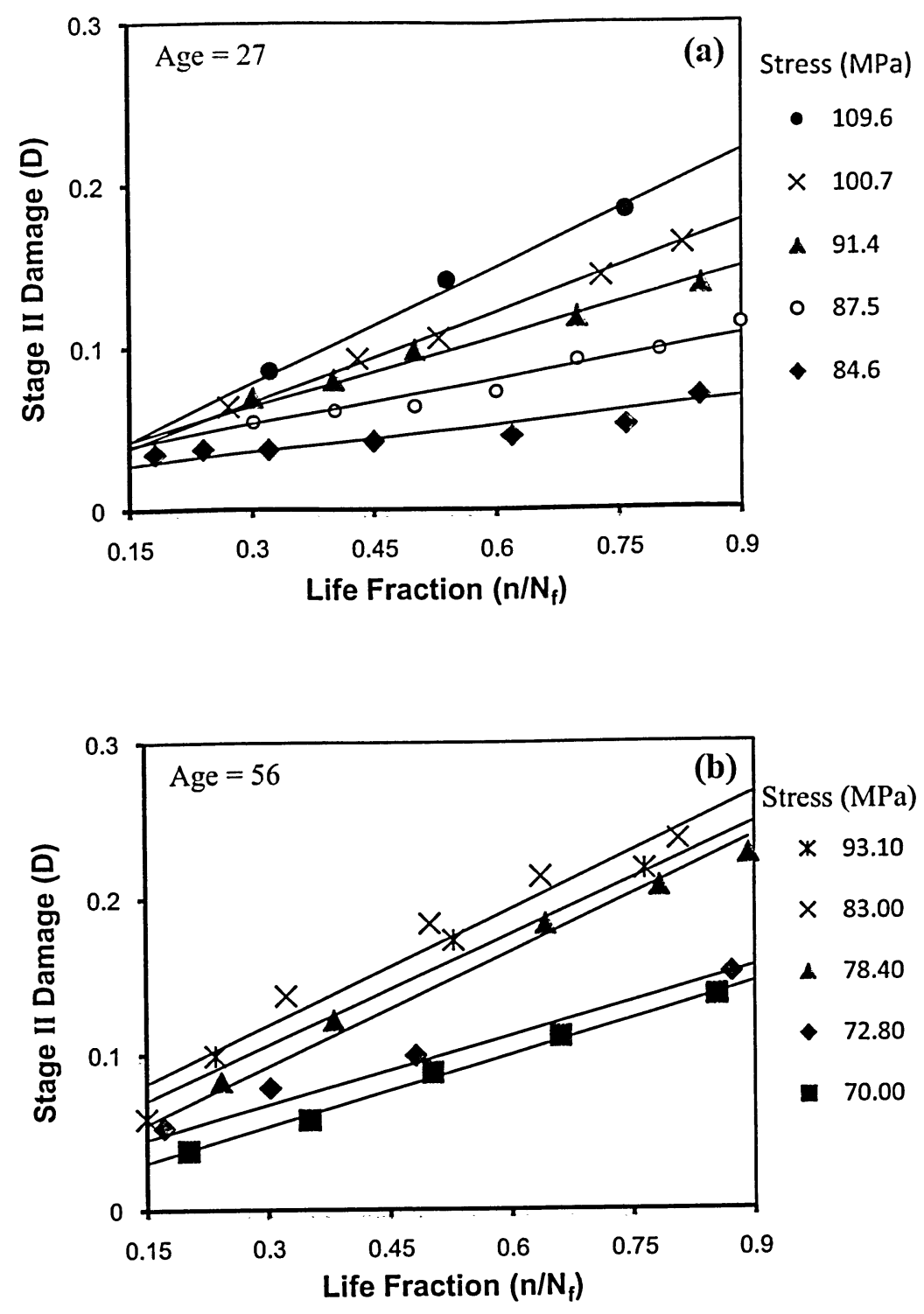

Fig. 5.9: Effect of stress magnitude on fatigue damage- $n / N_{f}$ diagrams for test specimens of a) 27-year-old and b) 56-year-old donor ages. 

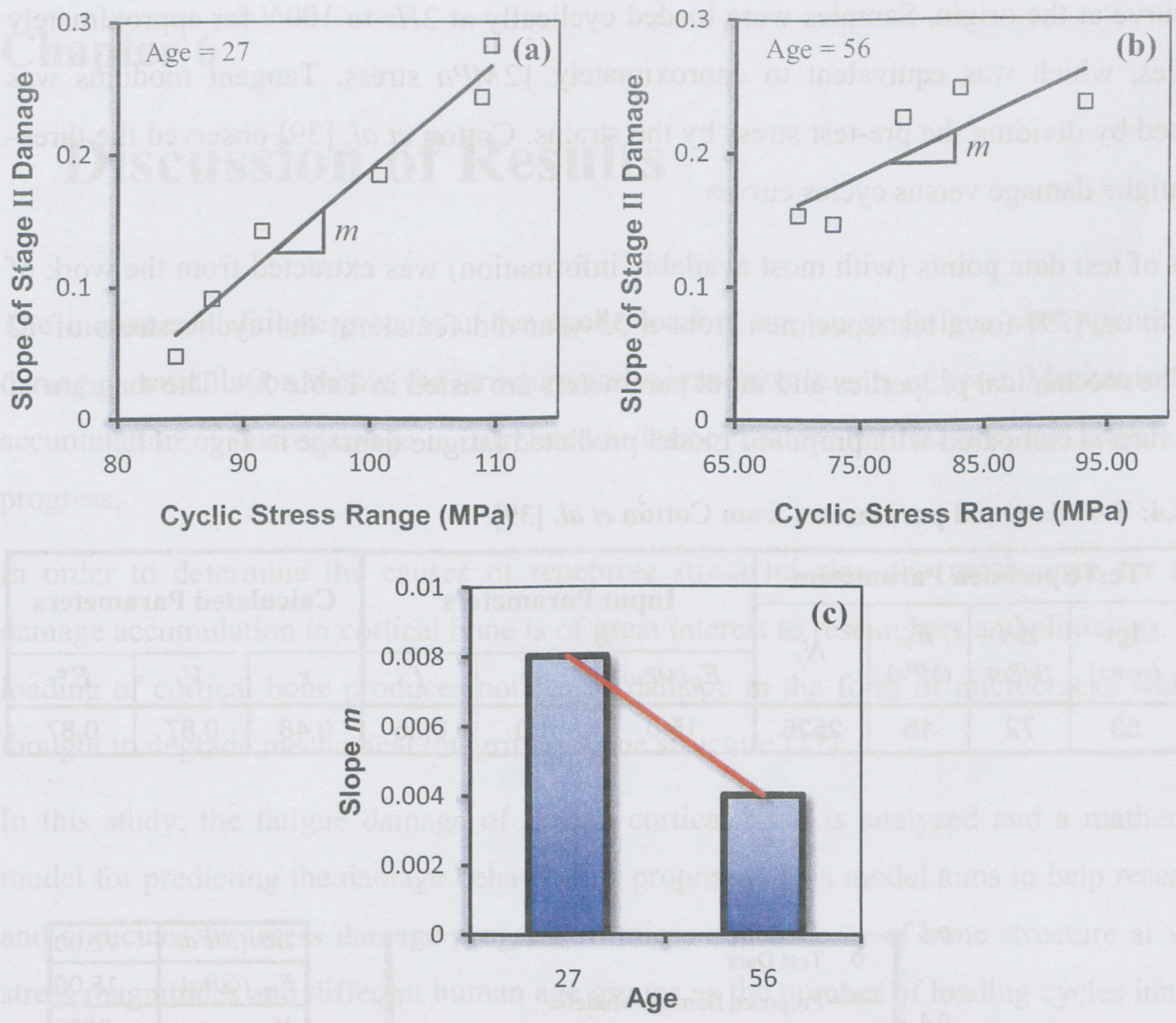

Fig. 5.10: Variation of slope $\boldsymbol{m}$ with cyclic stress magnitudes for cortical bone test specimens from donor age of a) 27 years, b) 56 years, c) presents slope $m$ vs. age diagram for above ages.

\subsection{Test Data by Cotton et al.}

Cotton et al. [39] cut samples from the mid-shaft cortex of the femur, in a sequential manner around the diaphysis. All samples were longitudinally oriented, with a rectangular cross section of approximately $8 \mathrm{~mm}^{2}$. Samples were fully immersed during testing, and the temperature was maintained at $37^{\circ} \mathrm{C}$ with Ringer's solution circulating between the test chamber and a larger reservoir. Thymol crystals were added to the bath to avert bacterial growth.

A fatigue-rated extensometer was used to determine strain. Before fatigue tests, each sample was tested to determine its undamaged tangent modulus $E^{*}$, which is the slope of the stress- 
strain curve at the origin. Samples were loaded cyclically at $2 \mathrm{~Hz}$ to $100 \mathrm{~N}$ for approximately 20 cycles, which was equivalent to approximately $12 M P a$ stress. Tangent modulus was calculated by dividing the pre-test stress by the strains. Cotton et al. [39] observed the threestage fatigue damage versus cycles curves.

One set of test data points (with most available information) was extracted from the work of Cotton et al. [39] for a test specimen from a 53-year-old female at the cyclic stress of 72 MPa. The mechanical properties and input parameters are listed in Table 5.4. The diagram of the test data is compared with proposed model predicted fatigue damage in Fig. 5.11.

Table 5.4: Test data and parameters from Cotton et al. [39].

\begin{tabular}{|c|c|c|c|c|c|c|c|c|c|c|}
\hline \multirow{3}{*}{$\begin{array}{c}\text { Data } \\
\#\end{array}$} & \multicolumn{4}{|c|}{ Test Specimen Parameters } & \multirow{2}{*}{\multicolumn{3}{|c|}{ Input Parameters }} & \multirow{2}{*}{\multicolumn{3}{|c|}{ Calculated Parameters }} \\
\hline & \multirow{2}{*}{$\begin{array}{c}\text { Age } \\
\text { (years) }\end{array}$} & \multirow{2}{*}{$\begin{array}{c}\Delta \sigma \\
(\mathrm{MPa})\end{array}$} & \multirow{2}{*}{$\begin{array}{c}E_{0} \\
(M P a)\end{array}$} & \multirow{2}{*}{$N_{f}$} & & & & & & \\
\hline & & & & & $E_{f}(M P a)$ & $\alpha$ & $f$ & $r$ & $V_{f}$ & $E_{f}^{*}$ \\
\hline 1 & 53 & 72 & 15 & 2526 & 15.0 & 1.0 & 0.35 & 0.48 & 0.87 & 0.87 \\
\hline
\end{tabular}

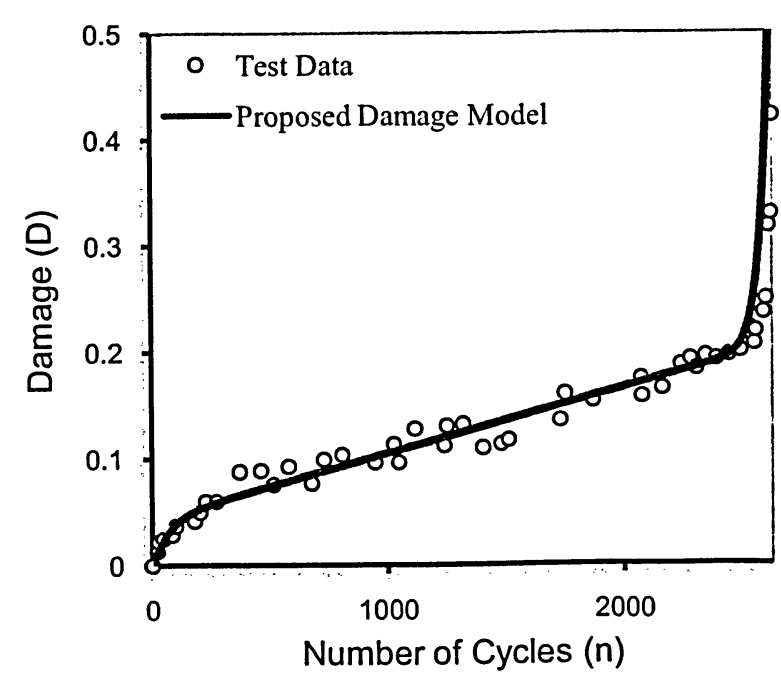

\begin{tabular}{|l|r|}
\hline$\triangle \sigma(M P a)$ & 72.00 \\
\hline$E_{0}(G P a)$ & 15.00 \\
\hline$N_{f}$ & 2626 \\
\hline$A g e$ & 53 \\
\hline \hline$E_{f}(G P a)$ & 15.00 \\
\hline$V_{f}$ & 0.87 \\
\hline$f$ & 0.35 \\
\hline
\end{tabular}

Fig. 5.11: Cotton et al. test data [39] compared with predicted damage based on the proposed fatigue damage model, Eq. (4.13). 


\section{Chapter 6}

\section{Discussion of Results}

The incremental failure process under cyclic loading known as fatigue corresponds to the damage accumulation within the structure and micro-constituents of bone. Mechanically, the accumulation of microdamage is correlated to loss of material stiffness as the fatigue cycles progress.

In order to determine the causes of repetitive stress injuries, the mechanism for fatigue damage accumulation in cortical bone is of great interest to researchers and clinicians. Cyclic loading of cortical bone produces noticeable damage in the form of microcracks which are thought to degrade mechanical integrity of bone structure [32].

In this study, the fatigue damage of human cortical bone is analyzed and a mathematical model for predicting the damage behaviour is proposed. This model aims to help researchers and clinicians to assess damage progress in micro-constituents of bone structure at various stress magnitudes and different human age groups as the number of loading cycles increases. Bone is a natural composite material consisting of secondary osteons as fibres and interstitial bone as matrix. Therefore, fatigue damage behaviour in composite materials has been used as a fundamental concept to develop the proposed damage model for cortical bone.

There have been some attempts to model the fatigue damage of cortical bone on the bases of stiffness loss [25], [38], [24], and [39], most of these damage models fail to correlate the mechanical and physiological properties of bone as an influential parameter to the accumulated damage. The proposed damage model successfully incorporates several influential bone mechanical and histological properties in to a mathematical relationship for cost effective and accurate prediction of fatigue damage of human cortical bone samples. These important parameters are osteon volume fraction, donor age, cyclic stress magnitude interfacial strength coefficient, mechanical properties of micro-constituents and more importantly damage evolution of bone structure over three stages of fatigue damage progress curve. 


\subsection{Evaluation Results and Diagrams}

In the previous chapter, the evaluation of the proposed damage model for tensile fatigue of human cortical bone (equation (4.13)) showed the capability of this model to predict fatigue damage for cortical bone samples. In most test data, the model passed through and connected the test data points with very high accuracy. The capability of the proposed model in accurately assessing fatigue damage in the micro-constituents of bone structure verifies the validity of the proposed damage model.

The Pattin et al. [24] test data of human cortical bone are of great importance as they clearly present the composite nature of cortical bone by having three stages of fatigue damage progress. The proposed damage model not only predicted the damage curves of Pattin's data with high accuracy by successfully presenting three stages of fatigue damage progress (see Figures 5.2 and 5.3), it also showed its capability of taking into account the effect of donor age as an influential factor (donor ages of 18,21 and 39 years). For the first time, the effect of age and histological properties of bone are employed in the fatigue damage model of cortical bone.

The proposed model strongly predicted the fatigue damage behaviour for the Griffin et al. [24] test data (Fig. 5.4). At the same age and bone secant modulus, applied stress is the main controlling factor changing the fatigue damage curve. The proposed model successfully shows this correlation by employing such factors as ratio $r$ (normalized applied cyclic stress) and cyclic stress range $\Delta \sigma$ throughout the mathematical model.

The Zioupos and Casinos [25] experimental data need a special attention as they include bone fatigue test data for two age groups of 27 years (young age) and 56 years (middle/old age). Bone specimens of these two age groups were fatigue tested under various cyclic stresses. The proposed damage model successfully predicted the damage for different stress magnitudes and age groups (see Figures 5.5-5.8). Osteon volume fraction is assumed equal for test specimens of the same donor age. In reality bone physiological and mechanical parameters other than age may slightly affect the volume fraction value. The variation of slope of D-n curves with stress magnitude is into account by the proposed damage model which shows very good agreement to the test data. 
The diagram of the data of Cotton at al. [39] also confirms the proposed model strength in prediction of the fatigue damage accumulation in human cortical bone (Fig. 5.11). The proposed damage model shows three stages of fatigue damage by utilising cortical bone mechanical and histological parameters such as: volume fraction, age, mechanical properties of constituents, cyclic stress amplitude and cement line interfacial strength coefficient.

\subsection{Comparison of Fatigue Damage Models}

In this section, the proposed damage model is benchmarked against three previously proposed fatigue damage models for human cortical bone. The models show decay of stiffness of bone micro-constituents as the number of fatigue cycles progress. The fatigue damage models discussed and compared in this chapter were developed based on the stiffness reduction in cortical bone as load cycle progressed. These models have been developed following the experimental results of fatigue tests on human cortical bone specimens. Thus, they all have some coefficients of curve fitting to test data which restricts the capability of these models for damage assessment of cortical bone and make this analysis costly and difficult to apply and use in clinical researches.

On the other hand, the compared models lack inclusion of important mechanical and histological properties of cortical bone specimens which are influential factors on fatigue damage response. The proposed damage model in this study effectively utilizes these factors to predict the fatigue damage response of cortical bone. These parameters are osteon volume fraction, donor age, cement line interfacial strength, cyclic stress amplitude and mechanical properties of cortical bone constituents. The proposed damage model also intuitively predicts the three phases of fatigue damage response common in most composites including cortical bone. An important advantage of the proposed damage model compared with other fatigue damage models of cortical bone is that all of its parameters are readily available from the mechanical and histological properties of cortical bone. This means that there is no coefficient to be obtained from costly experiments or estimated from curve fitting techniques. 


\subsubsection{Damage Model by Pattin et al.}

Pattin et al. [24] developed a damage assessment model to evaluate fatigue of cortical bone under tensile loading. They found that secant modulus degradation for tests conducted under tensile loading varied linearly with life cycles. The rate of modulus degradation was higher for specimens cycled at higher effective strain ranges. Extensive reduction occurred during the first quarter of fatigue life. Modulus degradation dropped and damage linearly increased over $70 \%$ of fatigue life in stage II of damage progress. A sharp decrease in modulus over the last $5 \%$ of fatigue life resulted in failure of cortical bone. The three stages of damage progress versus fatigue cycles are formulated as:

$$
1-\frac{E(n)}{E_{0}}=\alpha_{0}+\alpha_{1}\left[\log \left(\frac{n}{N_{f}}\right)\right]
$$

where $\alpha_{0}, \alpha_{1}$ : dimensionless parameters for each tensile fatigue test dependent on the effective strain range at which each test was performed,

$E(n)$ : secant modulus at cycle $\mathrm{n}, \mathrm{GPa}$,

$E_{0}$ : maximum secant modulus, $\mathrm{GPa}$,

$n$ : cycle number,

$N_{f}$ : fatigue life.

The least squares fit of $\alpha_{0}$ and $\alpha_{1}$ as functions of effective strain range $\left(\Delta \sigma / E^{*}\right)$ are [24]:

$$
\begin{aligned}
& \alpha_{0}=300.33\left(\frac{\Delta \sigma}{E^{*}}-0.002688\right) \\
& \alpha_{1}=247.70\left(\frac{\Delta \sigma}{E^{*}}-0.002951\right)
\end{aligned}
$$

where $E^{*}$ is the initial modulus of elasticity prior to fatigue testing,

Substituting equations (6.2) and (6.3) into equation (6.1), the damage model for cortical bone under tensile loading results in:

$$
D=300.33\left(\frac{\Delta \sigma}{E^{*}}-0.002688\right)+247.70\left(\frac{\Delta \sigma}{E^{*}}-0.002951\right)\left[\log \left(\frac{n}{N_{f}}\right)\right]
$$

where $D=\left(1-E(n) / E_{0}\right)$ and is damage due to stiffness degradation. 
Pattin's fatigue damage model was found to be in good agreement with conducted experimental fatigue data of age groups of 18, 21 and 39 (see Fig. 6.1). This model, however, fails to take into account the bone mechanical and physiological properties such as bone density, remodelling effect, age-related volume fraction of osteons and cement lines interfacial strength.

Figures 6.1-6.3 compare the results of the proposed damage model with the Pattin et al.'s model. Both models are relatively in good agreement at donor age of 18. As donor age increases, Pattin's model at different cyclic stresses show a more deviation from experimentally obtained damage-cycles data. It is also noticeable that Pattin's model fails to follow three stages of damage progress as number of cycles increases.

In Figures 6.2 and 6.3, the deviation of Pattin's damage model from two other sets of experimental data reported by Cotton et al. [39] and Griffin et al.[32] is more pronounced.

The proposed damage model, however, in Figures 6.1-6.3 shows very good agreement with experimental fatigue damage data reported by Pattin et al. [24], Cotton et al. [39] and Griffin et al.[32] for various cyclic stress magnitudes and donor ages. 


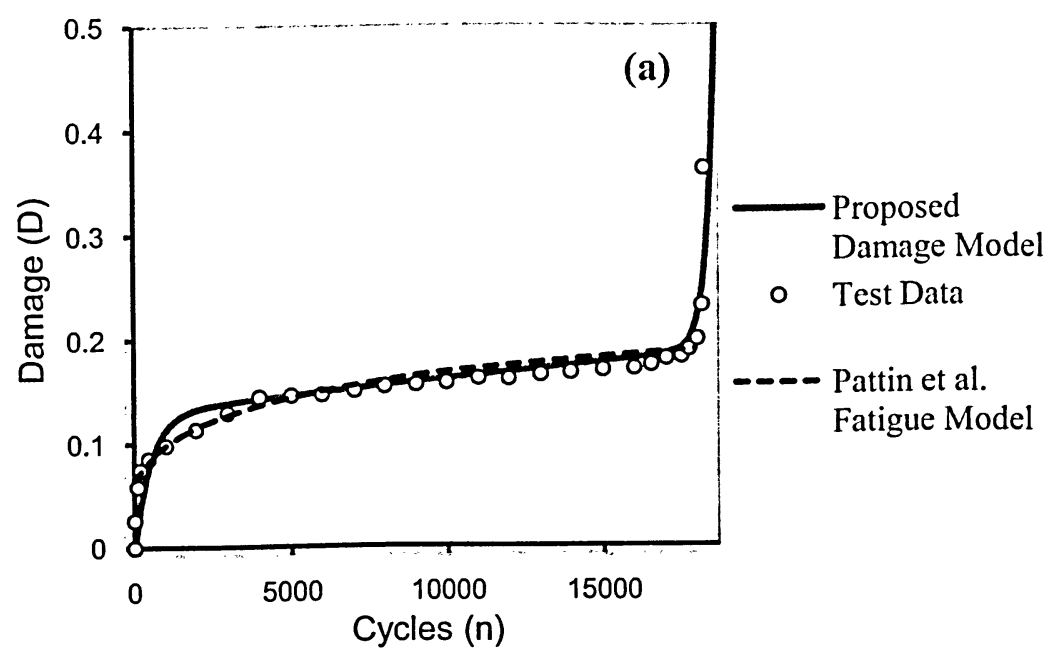

\begin{tabular}{|l|r|}
\hline$\triangle \sigma(M P a)$ & 70.70 \\
\hline$E^{*}(G P a)$ & 21.38 \\
\hline$N_{f}$ & 18658 \\
\hline Age & 18 \\
\hline
\end{tabular}

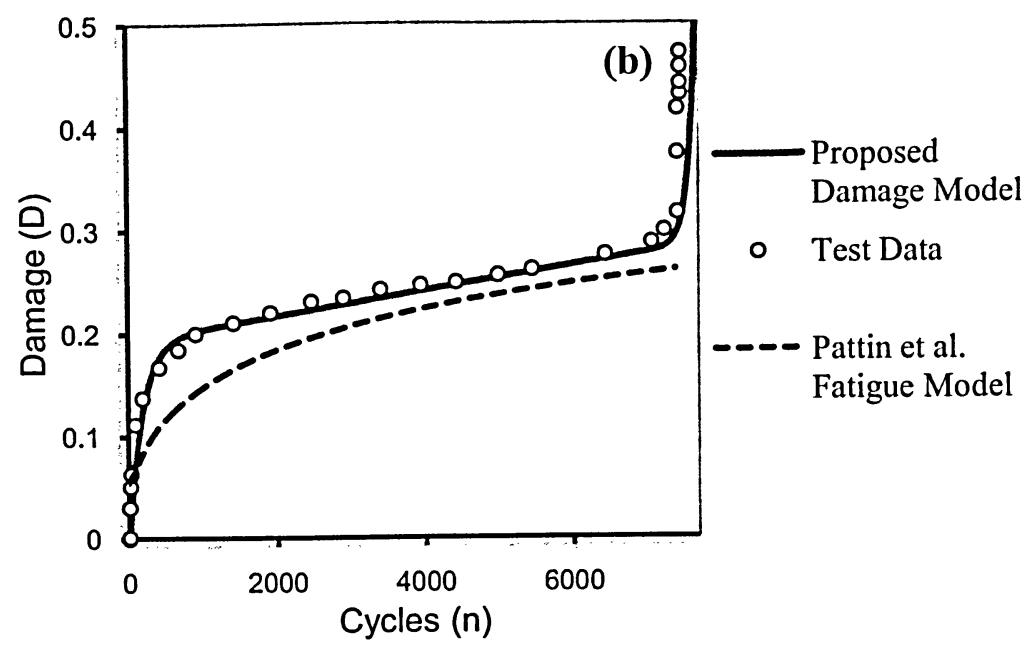

\begin{tabular}{|l|r|}
\hline$\Delta \sigma(M P a)$ & 82.30 \\
\hline$E^{*}(G P a)$ & 23.20 \\
\hline$N_{f}$ & 7688 \\
\hline Age & 21 \\
\hline
\end{tabular}

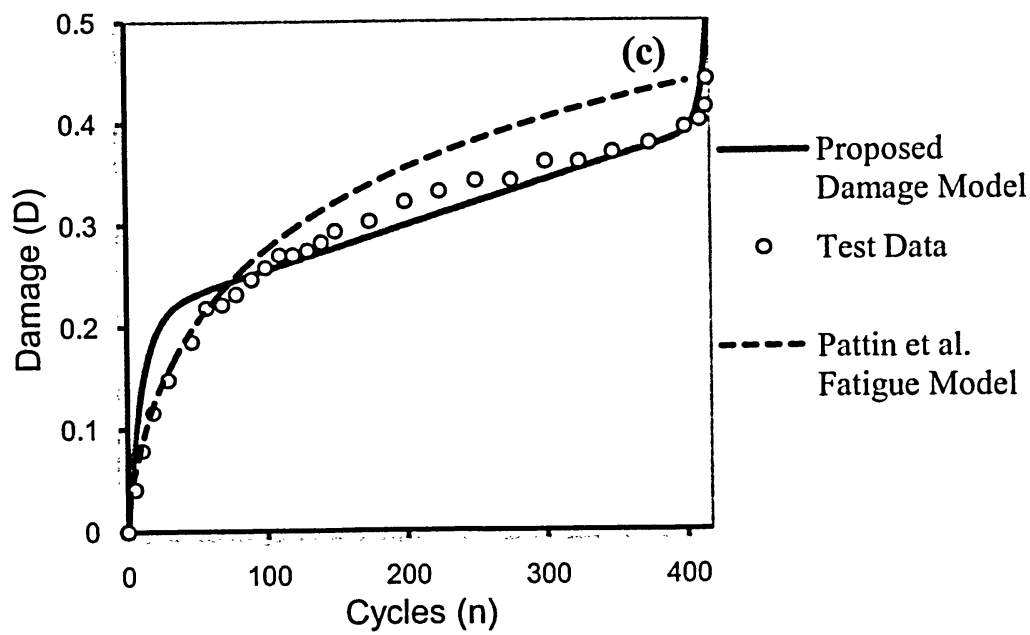

\begin{tabular}{|l|r|}
\hline$\triangle \sigma(M P a)$ & 83.00 \\
\hline$E^{*}(G P a)$ & 20.03 \\
\hline$N_{f}$ & 417 \\
\hline Age & 39 \\
\hline
\end{tabular}

Fig. 6.1: Proposed damage model is compared to fatigue model of Pattin et al. using their test data in [24]. 


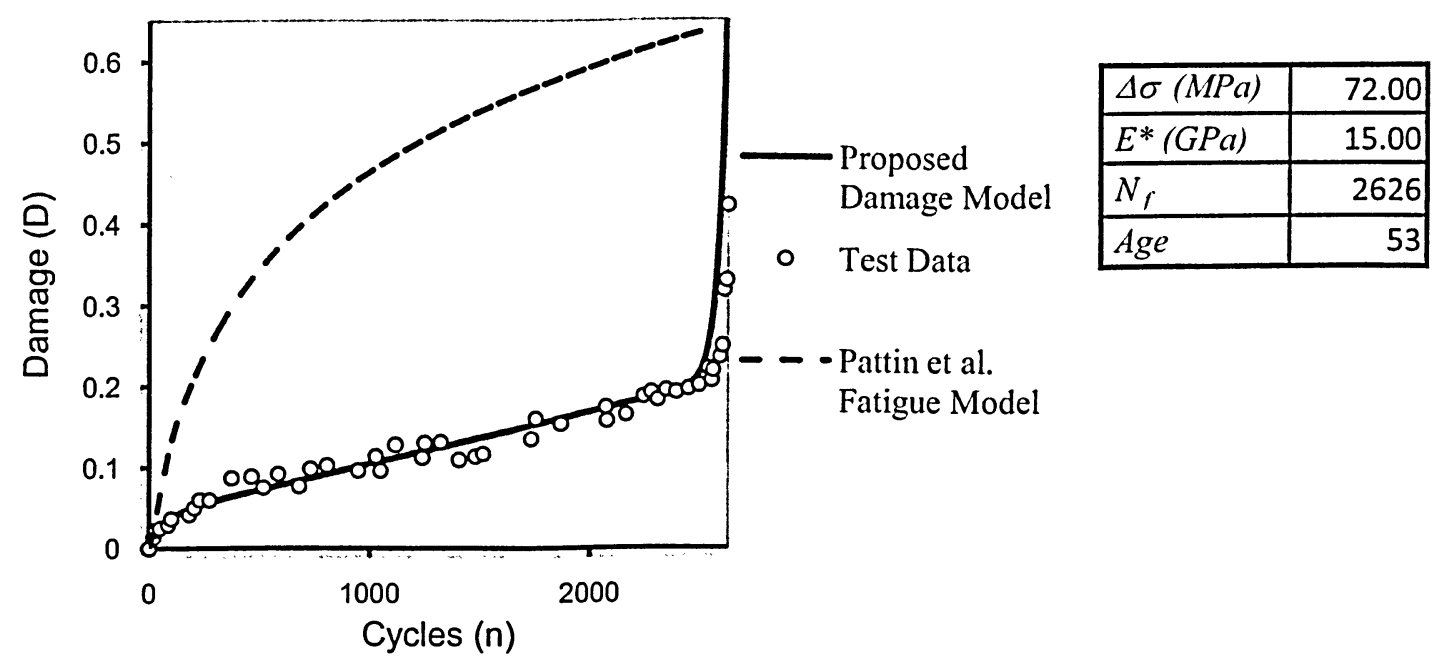

Fig. 6.2: Proposed damage model is compared to fatigue model of Pattin et al. in [24] using test data from Cotton et al. [39].

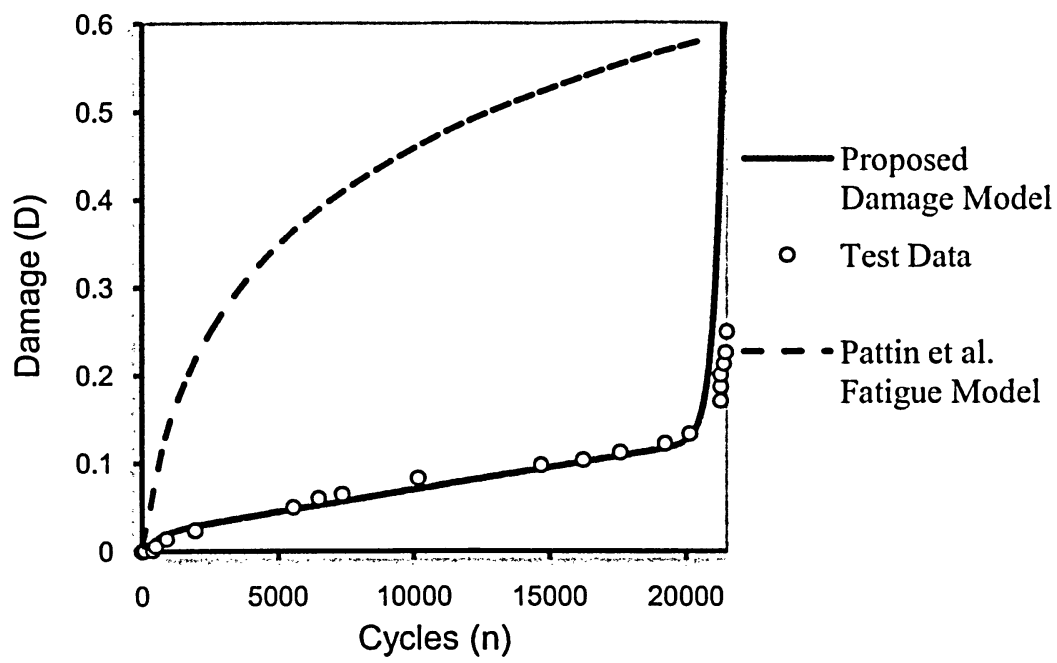

\begin{tabular}{|l|r|}
\hline$\Delta \sigma(M P a)$ & 69.20 \\
\hline$E^{*}(G P a)$ & 15.90 \\
\hline$N_{f}$ & 21500 \\
\hline Age & 50 \\
\hline
\end{tabular}

Fig. 6.3: Proposed damage model is compared to fatigue model of Pattin et al. in [24] using test data from Griffin et al. [32]. 


\subsubsection{Damage Model by Zioupos et al.}

Zioupos and Casinos [25] postulated that the development of damage in human cortical bone can be empirically described by exploiting the fact that $\mathrm{D}$ (Damage) is approximately a linear function of the fractional lifetime $n / N_{f}$ up to $n / N_{f}=0.90$. They found that a linear regression between D and $n / N_{f}$ (where $n / N_{f} \leq 0.9$ ) produced a line that intercepted the right-hand axis of diagram D versus $n / N_{f}$ at $D_{t}$ :

$$
D(n)=D_{t}\left(n / N_{f}\right)
$$

The relation between the damage $D_{t}$ with the imposed stress was expressed by:

$$
D_{t}=L \sigma^{M}
$$

An empirical relationship between $\sigma$ and $N_{f}$ is also given as [25]:

$$
N_{f}=F \sigma^{-\beta}
$$

By substituting equations (6.5) and (6.6) into (6.7), Zioupos and Casinos [25] proposed a damage model as:

$$
D(n)=L F^{-1} \sigma^{M+\beta} n
$$

Equation (6.8) provides a simple expression of fatigue damage as a function of the applied cyclic stress and number of fatigue cycles. Table 6.1 tabulates the calculated values for the constants $L, M, F$ and $\beta$.

Table 6.1: Constants of the equation (6.8) by Zioupos and Casinos [25].

\begin{tabular}{lcccc}
\hline & $\boldsymbol{L}$ & $\boldsymbol{M}$ & $\boldsymbol{F}$ & $\boldsymbol{\beta}$ \\
\hline 27-year-old female & $10^{-8.36}$ & 3.85 & $10^{62}$ & 29.5 \\
56-year-old female & $10^{-3.51}$ & 1.51 & $10^{36.5}$ & 17.8 \\
Pooled human data & $10^{-5.00}$ & 2.20 & $10^{41.8}$ & 19.7 \\
\hline
\end{tabular}

Zioupos fatigue damage model is highly dependent on experimental coefficients of $L, M, F$ and $\beta$. This in turn diminishes the capability of such damage model in assessing of damage in cortical bones with different mechanical properties under various stress magnitudes as obtaining coefficients of their model is costly and difficult. 

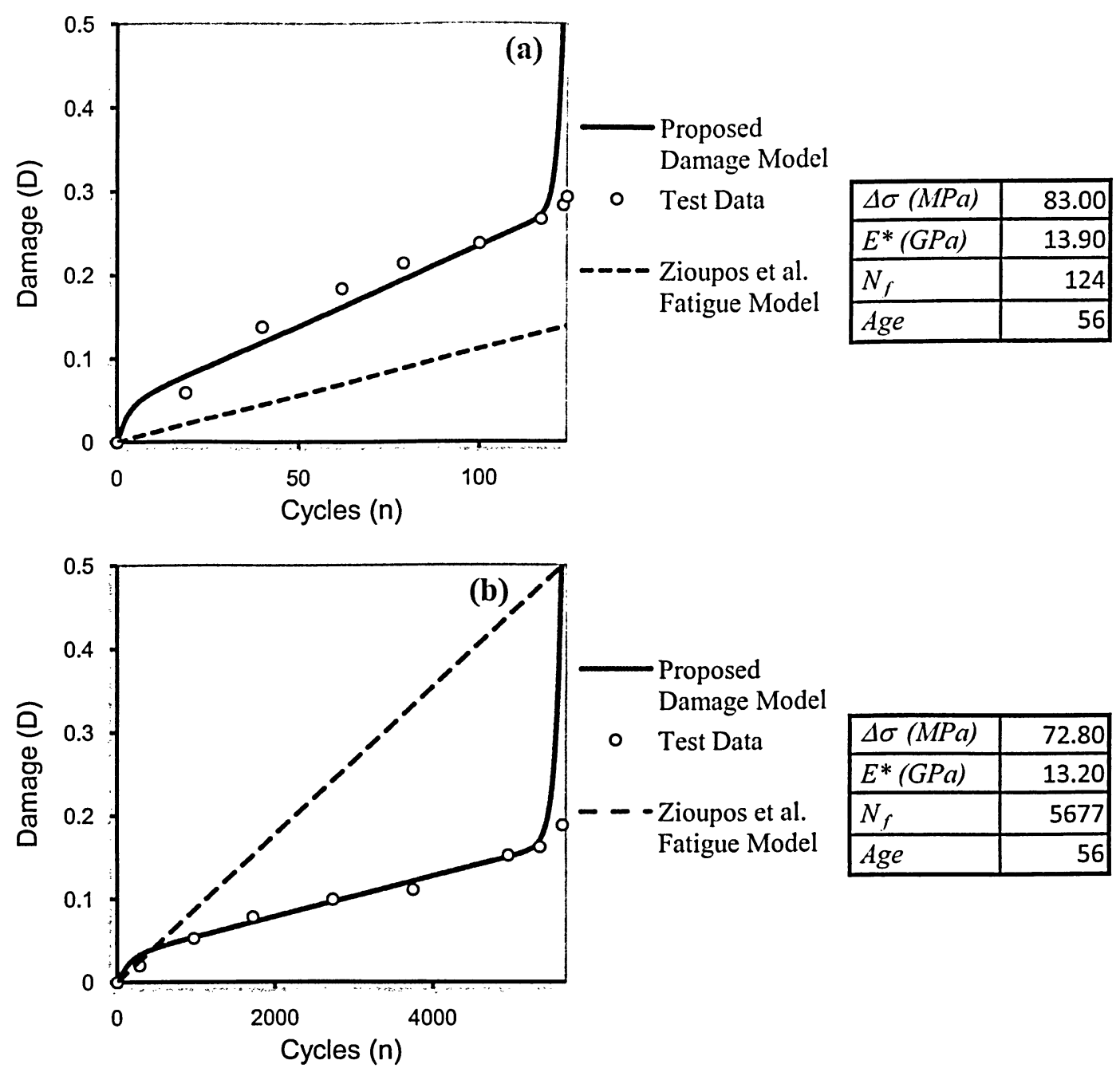

\begin{tabular}{|l|r|}
\hline$\triangle \sigma(M P a)$ & 72.80 \\
\hline$E^{*}(G P a)$ & 13.20 \\
\hline$N_{f}$ & 5677 \\
\hline Age & 56 \\
\hline
\end{tabular}

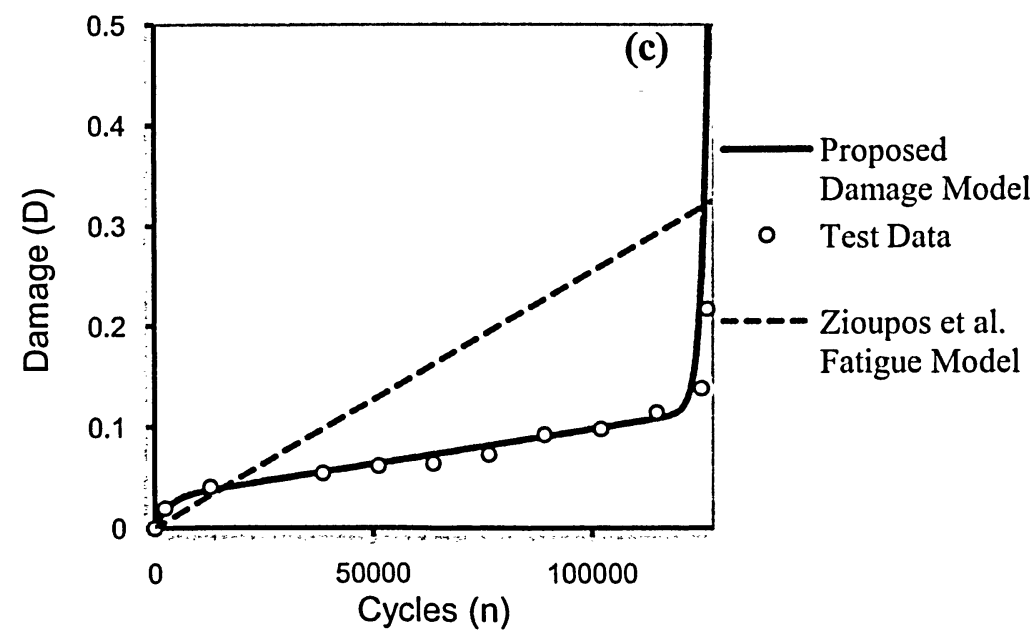

\begin{tabular}{|l|r|}
\hline$\triangle \sigma(M P a)$ & 87.50 \\
\hline$E^{*}(G P a)$ & 14.90 \\
\hline$N_{f}$ & 127590 \\
\hline Age & 27 \\
\hline
\end{tabular}

Fig. 6.4: Proposed damage model is compared to fatigue model of Zioupos and Casinos using their test data in [25]. 


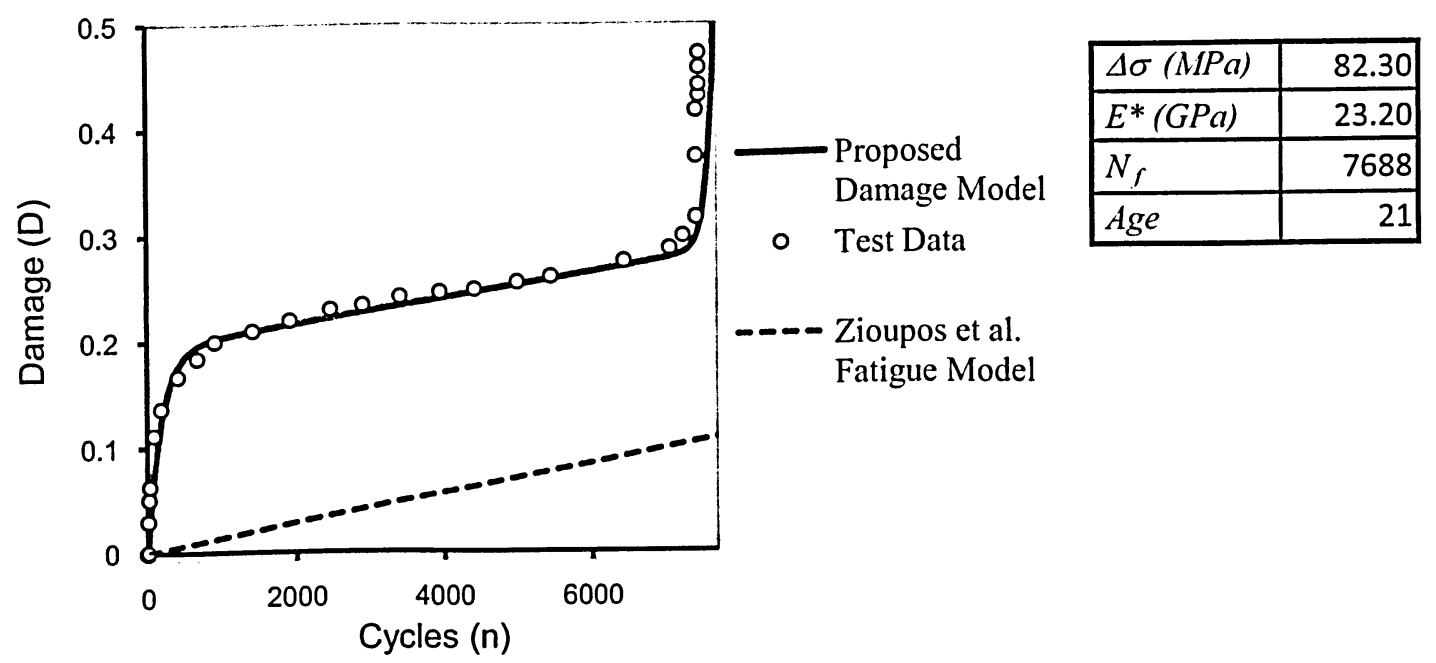

Fig. 6.5: Proposed damage model is compared to fatigue model of Zioupos and Casinos in [25] using test data from Pattin et al. [24].

Fig. 6.4 compares the results of the proposed damage model, fatigue damage model of Zioupos and Cosinos [25] and the experimental data from Zioupos and Cosinos [25]. They tried to model the damage response of cortical bone with a linear equation as the majority of the D-n curve is linear. However, the factors affecting the damage response are not available in their model which leads to poor results in predicting the fatigue response. This is more pronounced in Fig. 6.5 when comparing Zioupos fatigue damage model with experimental results from others.

\subsubsection{Damage Model by Griffin et al.}

The theory behind the fatigue damage model by Griffin et al. [32] is similar to that of the proposed model in this thesis. Griffin et al. [38] assumed that cortical bone could be modelled as a fibre-matrix composite. The fibres were considered complete osteons, and the matrix was the interstitial bone, composed of primary bone and remodelled osteon fragments. They also assumed that damage could be partitioned into components that independently affect the fibres and the matrix and the "damaged modulus" could be obtained from an isostrain rule of mixtures similar to that used in modeling ceramic matrix composites:

$$
E=E_{m} V_{m}\left(1-D_{m}\right)+E_{f} V_{f}\left(1-D_{f}\right)
$$


Griffin et al. [32] postulated that similar to ceramic matrix composites loaded in tension, the loss of stiffness is caused primarily by cracks that form in the interstitial matrix. They found that the initial damage is caused by a system of microcracks in the matrix. The number of these cracks does not increase indefinitely but approaches a limit known as the saturation damage state. They believed these cracks were self-limiting in length. Therefore, damage to the matrix evolves as:

$$
\frac{d D_{m}}{d n}=K_{1}\left(D_{s}-D_{m}\right)
$$

Term $D_{m}$ represents the matrix damage, $n$ is the cycle number, $K_{l}$ is the parameter of the rate of matrix damage, and $D_{s}=K_{2} \sigma_{a}^{q}$ represents the saturation level of matrix damage as functions of applied stress $\sigma_{a}$ and material constants of $K_{2}$ and $q$.

Griffin et al. [32] further presumed that matrix damage results in load transfer from the matrix to the osteons and should eventually lead to the tensile failure of the osteons. They postulated that as the osteons break, the load is further redistributed to neighbouring osteons, producing positive feedback and resulting in osteon failure at an ever increasing rate. Therefore, the fibre damage, $D_{f}$ was defined as:

$$
\frac{d D_{f}}{d n}=\left(K_{3} D_{s}-K_{4} D_{m}\right)
$$

where $K_{3}$ : coefficient of the rate of osteonal damage,

$K_{4}$ : parameter of the damage due to interaction of the osteon and matrix.

Griffin, based on equations (6.10) and (6.11), proposed $D_{m}$ and $D_{f}$ as

$$
\begin{aligned}
& D_{m}=K_{2} \sigma_{a}^{q}\left[1-\exp \left(-K_{1}\left(\frac{n}{N_{f}}\right)\right)\right] \\
& D_{f}=\frac{K_{2} K_{4} \sigma_{a}^{q}}{K_{1}+K_{3}}\left[K_{3} \exp \left(-K_{1}\left(\frac{n}{N_{f}}\right)\right)+K_{1} \exp \left(K_{3}\left(\frac{n}{N_{f}}\right)\right)-\left(K_{1}+K_{3}\right)\right]
\end{aligned}
$$

Combining equations (6.9), (6.12) and (6.13) one can calculate secant modulus drop and consequently bone fatigue damage at each load cycle up to the fatigue life of the specimen. 
Griffin et al. [32] listed the values for the constants of the equations (6.12) and (6.13) by curve fitting the data of Pattin et al. [24] but did not provide a method to approximately estimate these constants if test results were not available.

A significant drawback of this damage model is the tediousness of the method to obtain coefficient required in equations (6.12) and (6.13). The existence of many constants in this model makes it inapplicable for many applications including clinical researches. In fatigue damage assessment of cortical bone, a damage model with readily available parameters from the mechanical and physiological properties of bone and bone donor information is very desirable.

Physiological parameters such as donor age, volume fraction of osteons, interfacial strength factor $f$ and more importantly the effect of cyclic stress magnitude were not taken into account in Griffin's model.

Fig. 6.6 shows the comparison of the damage model of Griffin et al. [32] and the proposed damage model in this work for the test data performed by Griffin et al. [32]. Their fatigue damage model has the advantage of predicting three stages of the fatigue behaviour seen in cortical bones. The proposed damage model shows a better agreement with experimental data. More specifically, the damage response predicted by the proposed damage model in the third stage follows the trend of the experimental results contrary to the results of the fatigue damage model by Griffin. The slope of the linear portion of the damage curve (stage II) is more accurately estimated by the proposed damage model as it is clearly noticeable in Fig. 6.6c.

Complication of using Griffin's model due to numerous coefficients avoids one to employ this model in assessing other sets of fatigue data from other laboratories. This is mainly due to variation of coefficients and experimental errors involved in obtaining these experimentally based constants. 


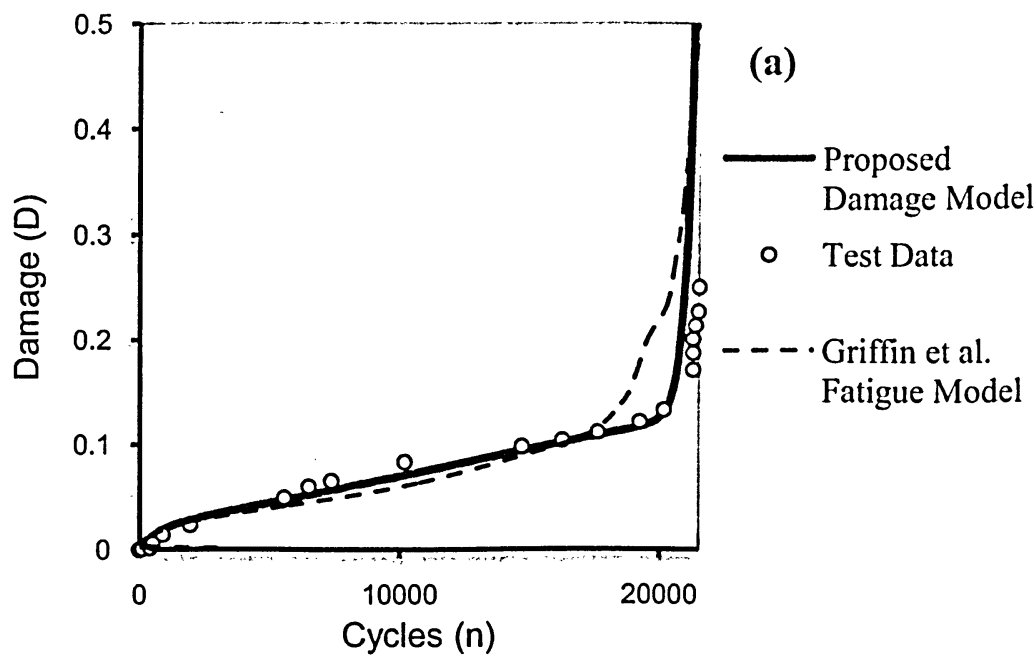

\begin{tabular}{|l|r|}
\hline$\Delta \sigma(M P a)$ & 69.20 \\
\hline$E^{*}(G P a)$ & 15.90 \\
\hline$N_{f}$ & 21500 \\
\hline Age & 50 \\
\hline
\end{tabular}

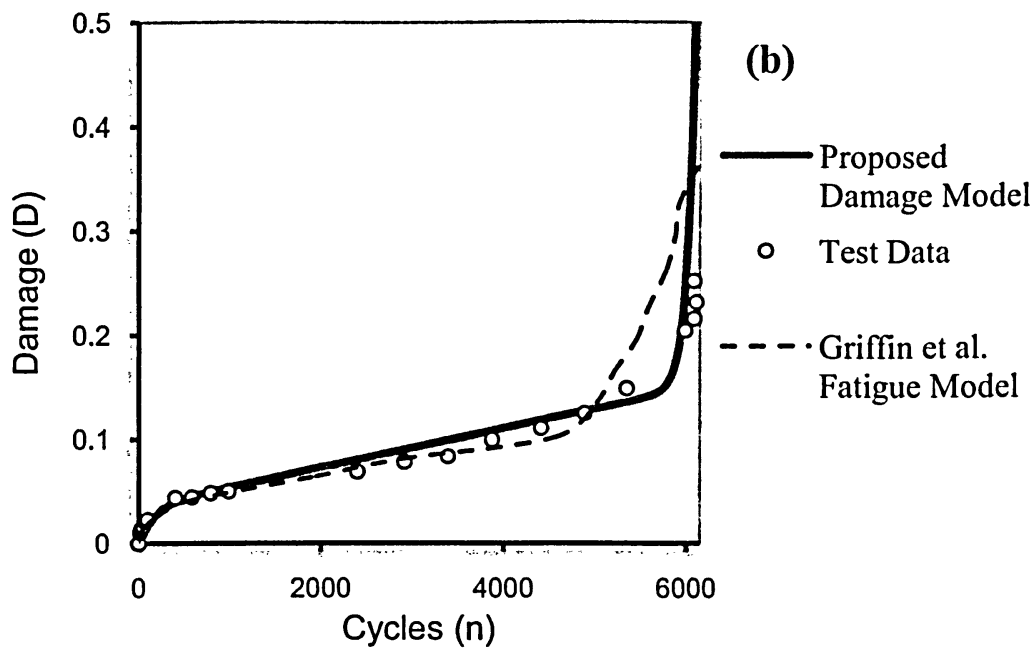

\begin{tabular}{|l|r|}
\hline$\Delta \sigma(M P a)$ & 73.50 \\
\hline$E^{*}(G P a)$ & 15.90 \\
\hline$N_{f}$ & 6150 \\
\hline$A g e$ & 50 \\
\hline
\end{tabular}

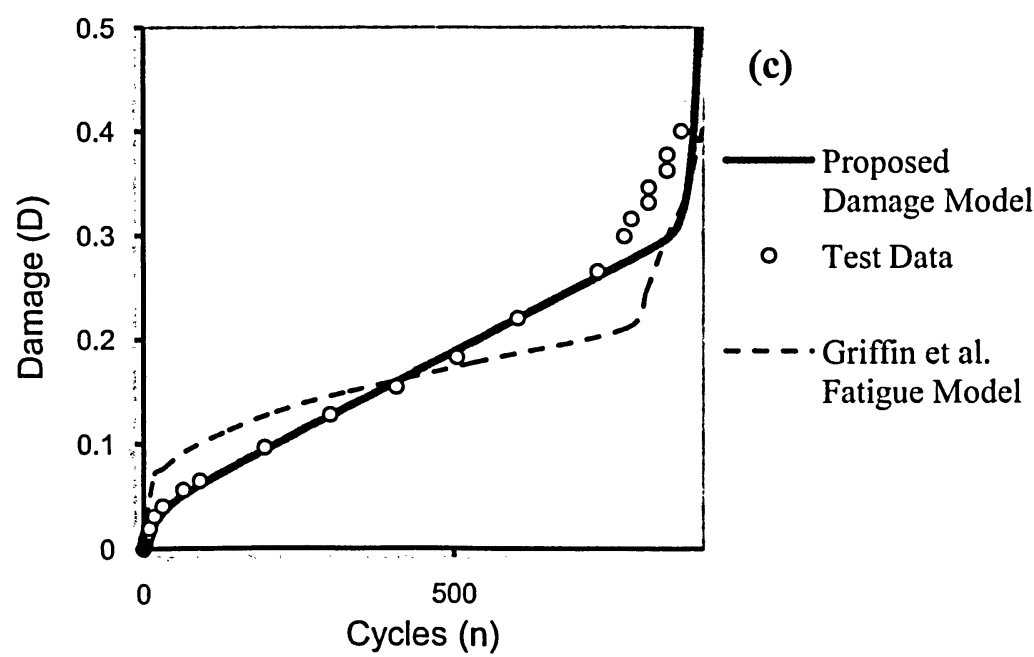

\begin{tabular}{|l|r|}
\hline$\Delta \sigma(M P a)$ & 80.90 \\
\hline$E^{*}(G P a)$ & 15.90 \\
\hline$N_{f}$ & 900 \\
\hline$A g e$ & 50 \\
\hline
\end{tabular}

Fig. 6.6: Proposed damage model is compared to fatigue model of Griffin $e t$ al. using their test data in [32]. 


\section{Chapter 7}

\section{Conclusion and Recommendations}

\subsection{Conclusions}

Fatigue damage accumulation of cortical bone was studied in this work. Stiffness loss in bone and bone constituents were used as a damage index to model the response of fatigue damage as number of cycles progressed. Bone is stronger in compression than in tension and as such a model for tensile fatigue response can unconservatively predict the shortest fatigue life of bone under real loading conditions. The proposed damage model in this thesis considered bone as a natural composite material consisting of Osteon fibres embedded in interstitial bone separated by cement lines. Predicted fatigue damage results were found in good agreement with many experimentally obtained damage results of human cortical bone. The proposed damage equation also showed a higher degree of success in damage assessment of cortical bone samples tested by different laboratories as compared to other earlier developed damage models.

The damage response of human cortical bone is found to be very similar to many composites as it shows three stages of fatigue life. Stage $I$ is characterized by a substantial stiffness drop due to microcrack initiation in the bone matrix. In stage II, microcracks turn into a gentle linear progress for most of the fatigue life. The interfacial strength of cement lines, separating osteons and interstitial bone, is found to be very influential on the slope of this linear part. In stage III, a sudden drop in the stiffness leads to a rapid accumulation of damage in cortical bone up to the failure point (100\% damage). Moreover, the overall damage was considered to be the collective damage to matrix and fibres. Damage functions for matrix and fibres were developed using sufficient mathematical functions as the cornerstone of the proposed damage model.

The proposed damage model, for the first time, successfully correlated the mechanical and histological properties of human cortical bone with damage accumulation of bone 
constituents. The parameters used in the proposed damage model are mostly readily available and very inexpensive to obtain. Contrary to other earlier damage models, which extensively were defined by curve-fitting coefficients obtained through costly and difficult experiments, the proposed damage model is constructed based on the physics of damage progress in micro-constituents of bone structure and employs few parameters. The proposed damage model includes both mechanical and histological parameters in cortical bone specimen such as osteon volume fraction, donor age, cyclic stress magnitude, secant modulus of osteons, cement line interfacial strength and other bone constituent mechanical properties.

A computer program was also developed to assess fatigue damage of cortical bone by the proposed damage model and evaluate the proposed model with experimental data extracted from the literature. The model was found to successfully predict the damage response of cortical bone specimens with high accuracy.

The proposed damage model proves to be very easy to implement as it uses few parameters which are not costly to obtain. It accurately predicts the fatigue damage response of human cortical bone under tensile fatigue loading. This makes the proposed damage model an effective tool for clinical researchers and pathologists to obtain fatigue damage information for human cortical bone samples.

\subsection{Recommendations}

One of the complications involved with damage analysis of cortical bone is the lack of material properties (e.g., stiffness) of the Osteons. Osteons exist in different mineral arrangements which greatly affect their stiffness values. Further future investigation is required to precisely and accurately compute this important property of the bone. Moreover, the effects of biological parameters (age, nutrition, physical activity) are crucial on damage assessment of cortical bones. Contrary to other materials with stable mechanical properties, these biological parameters greatly affect the mechanical properties of cortical bone. For example, the effect of age was studied and implemented on the volume fraction of osteons and cement-line interfacial strength. Further work is required to extend this work to other mechanical and histological properties for more accurate assessment of fatigue damage response of human cortical bone. 
Remodelling phenomenon in live cortical bone tissues affects the quality or density of bone and volume fraction of secondary osteons. Conducting an experimental study on remodelling phenomenon in live tissues is difficult and costly but important and essential. It is worthy to control physical activities (physiological loads) applied on live bone tissues and evaluate damage induced by daily physical activities. Such investigations are presently in the stage of animal testing and it is required to be extended for human bone in future. The present thesis aims a future extension of the proposed damage model for live human cortical bone tissues involving both biological and mechanical parameters. 


\section{Appendix A}

\section{Computer Code for Model Evaluation}

The following computer program (Maple version 10.0) was used to assess the proposed damage model in this thesis with the experimental data (listed in Appendix B).

$* * * * * * * * * *$ Program code starts here $* * * * * * * * * *$

Data points stored in a text file with the name fileName will be read to 'TestData' variable. The text file has two columns of numbers; first column is $\mathbf{n}$ (cycle) and the second is $D$ (Damage). However, the first four numbers are Stress Range $(\Delta \sigma, \mathrm{Pa})$, Initial Modulus of Elasticity (E0, Pa), Fatigue Life (Nf) and Age.

$>$ restart; with(plots); with(plots);

$>$ fileName := "Cotton53-72";

> TestData $:=$ readdata (cat(fileName, ".txt"), 2);

Storing bone specimen mechanical properties into their respective variables. Here $\sigma$ represents cyclic stress range.

$>\operatorname{sigma}:=\operatorname{op}(1$, TestData $)[1] /(0.1 \mathrm{e} 7)$;

$E[0]:=\operatorname{op}(1$, TestData $)[2] /(0.1 \mathrm{e})$;

$\mathrm{N}[\mathrm{f}]:=\mathrm{op}(2$, TestData $)[1]$;

Age :=op (2, TestData) [2] ;

Storing the rest of the values to "tData" and plotting the points into "ptl1" variable.

$>$ tData $:=[o p(3 \ldots$ nops (TestData), TestData) ]; 
$>$ plt1 := pointplot(tData) ;

Calculating the graph maximum and minimum scale for the damage axis.

$>$ upLimit $:=$ evalf [1] (op (nops (tData), tData)[2])+.2;

uplimit := 'if'(upLimit >1.0, 1.0, upLimit);

Calculating the proposed damage model parameters or inputting them from previous calculations. ' $C$ ', here, is ' $E^{*} f$ ' (fibres contribution to stiffness drop) and ' $s$ ' represents ' $f$ ' (interfacial strength parameter) in the proposed damage model.

$>r:=100 *$ sigma/E $[0]$;

$>$ alpha $:=1.0 ; \mathrm{V}[f]:=\operatorname{alpha}(1-\exp (-4 *(1 / 100) *$ Age $)-$ $.20 * 10^{\wedge}(-3) *$ Age) ;

$>E[f]:=19000$;

$s:=.4 ;$

$C:=E[f] * V[f] / E[0]$

Executing the proposed damage model and plotting the values against the test data.

$>\mathrm{Dg}:=(1-\mathrm{C}) *_{r} *\left((1-\mathrm{s}) *\left(1-\exp \left(-(1 / 2) * \operatorname{sigma}{ }^{\mathrm{n}} / \mathrm{N}[f]\right)\right)\right.$ $+s * n / N[f])+C *(r * s * n / N[f]+(1-s) * \exp (s i g m a *(n / N[f]-$ 1)) );

$>$ plt2 := plot (Dg, $n=0 \ldots N[f], y=0 \ldots$ uplimit);

$>$ display $(\{p l t 1$, plt2\}, labelfont $=$ [TIMES, BOLD, 12], labeldirections = [horizontal, vertical], labels $=[" n$ (Number of Cycles)", "D (Damage)"], thickness = 2 , symbolsize $=15$, tickmarks $=[10,10])$; 
Finally exporting the results of model assessment to a new text file for later analysis.

$>$ modelData $:=[[0,0]]$;

$>$ for $j$ by round $((1 / 100) * N[f])$

to $N[f]-1$

do

modelData $:=[o p(m o d e l D a t a),[j, \operatorname{eval}(\mathrm{Dg}, \mathrm{n}=$ $1.0 * j)]]$

end do;

$>$ modelData $:=[o p(m o d e l D a t a),[N[f]$, eval $(\mathrm{Dg}, \mathrm{n}=$ $\mathrm{N}[\mathrm{f}])]$ ] ;

$>$ writedata (cat(fileName, "-Model.txt"), modelData);

$* * * * * * * * * *$ Program code ends here $* * * * * * * * * *$ 


\section{Appendix B}

\section{Test Data}

In this appendix, all the test data used in the assessment of the proposed damage model are tabulated and presented for reference and possible future use. There has been a great effort to extract these data through personal communications with laboratories and from the original literature as accurately as possible. Tests were performed on human cortical bone of different age donors. They were also different in initial bone modulus of elasticity $\left(E_{0}\right)$, magnitude of applied cyclic stress range $(\Delta \sigma)$ and finally the number of cycles to failure $\left(N_{f}\right)$. Tables of data provide stiffness reduction damage as number of fatigue cycles progressed.

Data of Zioupos and Casinos [25].

\begin{tabular}{|c|c|c|c|c|c|}
\hline $\begin{array}{l}\text { Donor Age } \\
\Delta \sigma(M P a)\end{array}$ & $\begin{array}{l}27 \\
84.6\end{array}$ & $\begin{array}{l}\text { Donor Age } \\
\Delta \sigma(\mathrm{MPa})\end{array}$ & $\begin{array}{l}27 \\
87.5\end{array}$ & $\begin{array}{l}\text { Donor Age } \\
\Delta \sigma(M P a)\end{array}$ & $\begin{array}{l}27 \\
91.4\end{array}$ \\
\hline$E_{0}(\mathrm{GPa})$ & 14.9 & $E_{0}(G P a)$ & 14.9 & $E_{0}(G P a)$ & 16.7 \\
\hline$N_{f}$ & 209480 & $N_{f}$ & 127590 & $N_{f}$ & 11863 \\
\hline$n$ (cycle) & $D$ (damage) & $n$ (cycle) & $D$ (damage) & $n$ (cycle) & $D$ (damage) \\
\hline 0 & 0 & 0 & 0 & 0 & 0 \\
\hline 16675 & 0.020399592 & 2360 & 0.01949961 & 1185 & 0.026639467 \\
\hline 38000 & 0.034439311 & 12695 & 0.040439191 & 3560 & 0.069358613 \\
\hline 50212 & 0.03701926 & 38481 & 0.054178916 & 4754 & 0.080338393 \\
\hline 67034 & 0.03701926 & 51266 & 0.061378772 & 5934 & 0.098698026 \\
\hline 94140 & 0.042239155 & 63833 & 0.063898722 & 8293 & 0.11849763 \\
\hline 129710 & 0.04547909 & 76554 & 0.072958541 & 10089 & 0.138597228 \\
\hline 158849 & 0.051718966 & 89211 & 0.092218156 & 10913 & 0.150356993 \\
\hline 177765 & 0.06899862 & 102034 & 0.09797804 & 11625 & 0.158636827 \\
\hline 200703 & 0.080458391 & 114691 & 0.114357713 & 11741 & 0.212215756 \\
\hline 204997 & 0.089338213 & 124974 & 0.138537229 & & \\
\hline 207448 & 0.099418012 & 126250 & 0.217375652 & & \\
\hline
\end{tabular}


Data of Zioupos and Casinos [25] continued

\begin{tabular}{|c|c|c|c|c|c|}
\hline Donor Age & 27 & Donor Age & 27 & Donor Age & 27 \\
\hline$\Delta \sigma(M P a)$ & 100.7 & $\Delta \sigma(M P a)$ & 104.8 & $\Delta \sigma(M P a)$ & 108.8 \\
\hline$E_{0}(\mathrm{GPa})$ & 17.3 & $E_{0}(G P a)$ & 15.1 & $E_{0}(G P a)$ & 15.4 \\
\hline$N_{f}$ & 402 & $N_{f}$ & 392 & $N_{f}$ & 87 \\
\hline$n$ (cycle) & $D$ (damage) & $n$ (cycle) & $D$ (damage) & $n$ (cycle) & $D$ (damage) \\
\hline 0 & 0 & 0 & 0 & 0 & 0 \\
\hline 32 & 0.019439611 & 39 & 0.024119518 & 9 & 0.026339473 \\
\hline 57 & 0.0400192 & 118 & 0.098698026 & 28 & 0.085558289 \\
\hline 109 & 0.06347873 & 196 & 0.177656447 & 47 & 0.141717166 \\
\hline 173 & 0.093178136 & 274 & 0.261534769 & 66 & 0.184676306 \\
\hline 213 & 0.105717886 & 297 & 0.291954161 & 86 & 0.239755205 \\
\hline 293 & 0.143817124 & 353 & 0.366532669 & & \\
\hline 333 & 0.16349673 & 385 & 0.415971681 & & \\
\hline 386 & 0.189656207 & 388 & 0.435891282 & & \\
\hline 398 & 0.212455751 & & & & \\
\hline
\end{tabular}


Data of Zioupos and Casinos [25] continued

\begin{tabular}{|c|c|c|c|c|c|}
\hline Donor Age & 27 & Donor Age & 27 & Donor Age & 56 \\
\hline$\Delta \sigma(\mathrm{MPa})$ & 109.6 & $\Delta \sigma(M P a)$ & 119.6 & $\Delta \sigma(M P a)$ & 70 \\
\hline$E_{0}(G P a)$ & 13.1 & $E_{0}(G P a)$ & 15.6 & $E_{0}(G P a)$ & 11.1 \\
\hline & 342 & $N_{f}$ & 13 & $N_{f}$ & 8053 \\
\hline$n$ (cycle) & $D$ (damage) & $n$ (cycle) & $D$ (damage) & $n$ (cycle) & $D$ (damage) \\
\hline 0 & 0 & 0 & 0 & 0 & 0 \\
\hline 41 & 0.024839503 & 1 & 0.019319614 & 405 & 0.014699706 \\
\hline 89 & 0.088078238 & 1 & 0.034799304 & 1619 & 0.038399232 \\
\hline 137 & 0.137877242 & 2 & 0.070918582 & 2825 & 0.057958841 \\
\hline 191 & 0.183116338 & 2 & 0.070918582 & 4049 & 0.088138237 \\
\hline 264 & 0.227335453 & 3 & 0.103797924 & 5320 & 0.111177776 \\
\hline 315 & 0.257094858 & 4 & 0.138837223 & 6870 & 0.137577248 \\
\hline 336 & 0.276474471 & 5 & 0.168836623 & 7424 & 0.147057059 \\
\hline \multirow[t]{9}{*}{339} & 0.307913842 & 6 & 0.188756225 & 7906 & 0.162596748 \\
\hline & & 7 & 0.216115678 & 7992 & 0.197936041 \\
\hline & & 8 & 0.239695206 & 8003 & 0.253674927 \\
\hline & & 9 & 0.272334553 & & \\
\hline & & 10 & 0.291714166 & & \\
\hline & & 11 & 0.311033779 & & \\
\hline & & 12 & 0.320273595 & & \\
\hline & & 13 & 0.333293334 & & \\
\hline & & 13 & 0.475370493 & & \\
\hline
\end{tabular}


Data of Zioupos and Casinos [25] continued

\begin{tabular}{|c|c|c|c|c|c|}
\hline $\begin{array}{l}\text { Donor Age } \\
\Delta \sigma(M P a)\end{array}$ & $\begin{array}{l}56 \\
70\end{array}$ & $\begin{array}{l}\text { Donor Age } \\
\Delta \sigma(M P a)\end{array}$ & $\begin{array}{l}56 \\
72.8\end{array}$ & $\begin{array}{l}\text { Donor Age } \\
\Delta \sigma(M P a)\end{array}$ & $\begin{array}{l}56 \\
78.4\end{array}$ \\
\hline$E_{0}(G P a)$ & 15.7 & $E_{0}(G P a)$ & 13.2 & $E_{0}(G P a)$ & 13.5 \\
\hline$N_{f}$ & 690 & $N_{f}$ & 5677 & $N_{f}$ & 942 \\
\hline$n$ (cycle) & $D$ (damage) & $n$ (cycle) & $D$ (damage) & $n$ (cycle) & $D$ (damage) \\
\hline 0 & 0 & 0 & 0 & 0 & 0 \\
\hline 35 & 0.020339593 & 284 & 0.019739605 & 104 & 0.052858943 \\
\hline 139 & 0.062758745 & 972 & 0.052798944 & 228 & 0.08297834 \\
\hline 209 & 0.088618228 & 1718 & 0.078538429 & 359 & 0.122037559 \\
\hline 332 & 0.117597648 & 2736 & 0.098878022 & 605 & 0.183236335 \\
\hline 484 & 0.14201716 & 3750 & 0.111177776 & 737 & 0.207595848 \\
\hline 609 & 0.162356753 & 4947 & 0.151916962 & 841 & 0.22799544 \\
\hline 664 & 0.172616548 & 5352 & 0.162236755 & 926 & 0.253314934 \\
\hline 685 & 0.188336233 & 5632 & 0.188396232 & 936 & 0.318233635 \\
\hline
\end{tabular}


Data of Zioupos and Casinos [25] Continued

\begin{tabular}{|c|c|c|c|c|c|}
\hline Donor Age & 56 & Donor Age & 56 & Donor Age & 56 \\
\hline$\Delta \sigma(M P a)$ & 83 & $\Delta \sigma(M P a)$ & 86.3 & $\Delta \sigma(M P a)$ & 93.1 \\
\hline$E_{0}(G P a)$ & 13.9 & $E_{0}(G P a)$ & 12.2 & $E_{0}(G P a)$ & 13.7 \\
\hline$N_{f}$ & 124 & $N_{f}$ & 339 & $N_{f}$ & 17 \\
\hline$n$ (cycle) & $D$ (damage) & $n$ (cycle) & $D$ (damage) & $n$ (cycle) & $D$ (damage) \\
\hline 0 & 0 & 0 & 0 & 0 & 0 \\
\hline 19 & 0.058558829 & 34 & 0.028139437 & 2 & 0.048179036 \\
\hline 40 & 0.13751725 & 95 & 0.062938741 & 4 & 0.099118018 \\
\hline 62 & 0.183536329 & 170 & 0.122457551 & 9 & 0.172796544 \\
\hline 79 & 0.213775724 & 224 & 0.151796964 & 13 & 0.218455631 \\
\hline 100 & 0.237295254 & 299 & 0.186176276 & 17 & 0.248455031 \\
\hline 117 & 0.266934661 & 326 & 0.197276054 & 17 & 0.253674927 \\
\hline 123 & 0.283254335 & 336 & 0.22451551 & & \\
\hline 124 & 0.293034139 & & & & \\
\hline
\end{tabular}


Data of Griffin et al. [32]

\begin{tabular}{|c|c|c|c|c|c|}
\hline Donor Age & 50 & Donor Age & 50 & Donor Age & 50 \\
\hline$\Delta \sigma(M P a)$ & 69.2 & $\Delta \sigma(M P a)$ & 73.5 & $\Delta \sigma(M P a)$ & 80.9 \\
\hline$E_{0}(G P a)$ & 15.9 & $E_{0}(G P a)$ & 15.9 & $E_{0}(\mathrm{GPa})$ & 15.9 \\
\hline$N_{f}$ & 21500 & $N_{f}$ & 6150 & $N_{f}$ & 900 \\
\hline$n$ (cycle) & $D$ (damage) & $n$ (cycle) & $D$ (damage) & $n$ (cycle) & $D$ (damage) \\
\hline 0 & 0 & 0 & 0 & 0 & 0 \\
\hline 8 & 0.000116669 & 13 & 0.010087374 & 2 & 0.003103103 \\
\hline 52 & 0.000116669 & 22 & 0.013242784 & 3 & 0.005255255 \\
\hline 90 & 0.000758349 & 32 & 0.014137231 & 4 & 0.008308308 \\
\hline 394 & 0.001341695 & 42 & 0.015950971 & 5 & 0.011636637 \\
\hline 499 & 0.005745953 & 62 & 0.020920121 & 7 & 0.015265265 \\
\hline 886 & 0.013796121 & 92 & 0.022733861 & 10 & 0.019444444 \\
\hline 1955 & 0.02321715 & 399 & 0.043753365 & 18 & 0.030930931 \\
\hline 5546 & 0.049526032 & 581 & 0.04425028 & 31 & 0.040365365 \\
\hline 6483 & 0.060317923 & 787 & 0.047852913 & 64 & 0.055880881 \\
\hline 7367 & 0.065130524 & 981 & 0.050014493 & 91 & 0.064414414 \\
\hline 10165 & 0.083097565 & 2395 & 0.068673651 & 195 & 0.096196196 \\
\hline 14686 & 0.097564533 & 2915 & 0.078015653 & 301 & 0.127977978 \\
\hline 16248 & 0.103981333 & 3387 & 0.083779867 & 407 & 0.15477978 \\
\hline 17608 & 0.111973166 & 3870 & 0.099581763 & 504 & 0.183458458 \\
\hline 19235 & 0.122123378 & 4407 & 0.110712659 & 602 & 0.220645646 \\
\hline 20151 & 0.133352778 & 4885 & 0.125098348 & 730 & 0.265615616 \\
\hline 21286 & 0.171270235 & 5346 & 0.148975113 & 773 & 0.299724725 \\
\hline 21286 & 0.187312236 & 5987 & 0.204654437 & 784 & 0.316016016 \\
\hline 21286 & 0.20064168 & 6087 & 0.215810178 & 812 & 0.331506507 \\
\hline 21380 & 0.213475281 & 6087 & 0.252432813 & 812 & 0.346246246 \\
\hline 21468 & 0.226308881 & 6107 & 0.231959916 & 842 & 0.362512513 \\
\hline \multirow[t]{2}{*}{21493} & 0.249730203 & & & 842 & 0.378028028 \\
\hline & & & & 864 & 0.400750751 \\
\hline
\end{tabular}


Data of Pattin et al.[24]

\begin{tabular}{|c|c|c|c|c|c|}
\hline Donor Age & 18 & $\begin{array}{l}\text { Donor Age } \\
\Lambda \sigma(M P a)\end{array}$ & $\begin{array}{l}21 \\
82.3\end{array}$ & $\begin{array}{l}\text { Donor Age } \\
\Delta \sigma(M P a)\end{array}$ & $\begin{array}{l}24 \\
110\end{array}$ \\
\hline$\Delta \sigma(M P a)$ & 70.7 & $\begin{array}{l}\Delta \sigma(M P a) \\
E_{0}(G P a)\end{array}$ & $\begin{array}{l}82.3 \\
19.261\end{array}$ & $\begin{array}{l}\Delta \sigma(I I P a) \\
E_{0}(G P a)\end{array}$ & 22.26 \\
\hline$E_{0}(G P a)$ & 19.98 & $E_{0}(G P a)$ & $\begin{array}{l}19.261 \\
7688\end{array}$ & $\mathrm{~N}_{f}$ & \\
\hline$N_{f}$ & 18658 & $N_{f}$ & 7688 & $N_{f}$ & 60 \\
\hline$n$ (cycle) & $D$ (damage) & $n$ (cycle) & $D$ (damage) & $n$ (cycle) & $D$ (damage) \\
\hline 1 & 0 & 0 & 0 & 0 & 0 \\
\hline 6 & 0.0260413 & 3 & 0.000905379 & 2 & 0.015977277 \\
\hline 115 & 0.0587961 & 12 & 0.030019528 & 3 & 0.04912125 \\
\hline 186 & 0.0743968 & 21 & 0.05059471 & 4 & 0.088443103 \\
\hline 453 & 0.0850159 & 31 & 0.063287769 & 5 & 0.133392508 \\
\hline 999 & 0.0982132 & 88 & 0.11200071 & 6 & 0.164849991 \\
\hline 1958 & 0.1133275 & 188 & 0.137014024 & 7 & 0.191248003 \\
\hline 2972 & 0.1290726 & 413 & 0.16722883 & 8 & 0.221232026 \\
\hline 3989 & 0.1440356 & 673 & 0.184218001 & 9 & 0.242588319 \\
\hline 4997 & 0.1453477 & 905 & 0.19971596 & 10 & 0.271240902 \\
\hline 5967 & 0.146135 & 1414 & 0.209817149 & 11 & 0.289774543 \\
\hline 6997 & 0.1498088 & 1920 & 0.219989348 & 12 & 0.302822652 \\
\hline 7956 & 0.1534827 & 2464 & 0.230569856 & 13 & 0.319119475 \\
\hline 8973 & 0.1553196 & 2894 & 0.23431564 & 14 & 0.335416297 \\
\hline 9942 & 0.1566317 & 3399 & 0.242197763 & 15 & 0.352831528 \\
\hline 10983 & 0.1616177 & 3942 & 0.245943547 & 16 & 0.37305166 \\
\hline 11947 & 0.1610928 & 4421 & 0.248961477 & 18 & 0.394549973 \\
\hline 12989 & 0.1650291 & 4993 & 0.255334635 & 20 & 0.410846796 \\
\hline 13950 & 0.1668966 & 5451 & 0.260962187 & 22 & 0.429948518 \\
\hline 14969 & 0.1692584 & 6433 & 0.275217468 & 24 & 0.447363749 \\
\hline 15961 & 0.1710953 & 7057 & 0.287608734 & 26 & 0.465347062 \\
\hline 16501 & 0.1739819 & 7231 & 0.299236641 & 29 & 0.482762294 \\
\hline 16985 & 0.1800175 & 7408 & 0.316119297 & 32 & 0.499609444 \\
\hline 17476 & 0.1818545 & 7408 & 0.374418605 & 35 & 0.514770105 \\
\hline 17707 & 0.1889398 & 7408 & 0.417575004 & 40 & 0.531066927 \\
\hline 17971 & 0.1981244 & 7446 & 0.431830286 & 44 & 0.546795668 \\
\hline 18134 & 0.2310091 & 7446 & 0.44196698 & 48 & 0.56252441 \\
\hline \multirow[t]{2}{*}{18180} & 0.3621308 & 7446 & 0.457731227 & 54 & 0.576584413 \\
\hline & & 7446 & 0.471986508 & 59 & 0.591176993 \\
\hline
\end{tabular}


Data of Pattin et al. [24] continued

\begin{tabular}{|c|c|c|c|c|c|}
\hline Donor Age & 39 & Donor Age & 39 & Donor Age & 43 \\
\hline$\Delta \sigma(M P a)$ & 55 & $\Delta \sigma(M P a)$ & 83 & $\Delta \sigma(M P a)$ & 63.7 \\
\hline$E_{0}(G P a)$ & 20.62 & $E_{0}(G P a)$ & 18.04 & $E_{0}(\mathrm{GPa})$ & 22.45 \\
\hline$N_{f}$ & 16331 & $N_{f}$ & 417 & $N_{f}$ & 23992 \\
\hline$n$ (cycle) & $D$ (damage) & $n$ (cycle) & $D$ (damage) & $n$ (cycle) & $D$ (damage) \\
\hline 0 & 0 & 0 & 0 & 0 & 0 \\
\hline 68 & 0.007936489 & 5 & 0.040539036 & 953 & 0.030765134 \\
\hline 159 & 0.017922306 & 10 & 0.079028845 & 1964 & 0.032788922 \\
\hline 466 & 0.026809748 & 18 & 0.116449493 & 2891 & 0.035007989 \\
\hline 695 & 0.045009078 & 29 & 0.148257044 & 3923 & 0.037031777 \\
\hline 1015 & 0.045221301 & 46 & 0.185588596 & 4824 & 0.036854252 \\
\hline 1531 & 0.045115999 & 57 & 0.218732598 & 5804 & 0.037031777 \\
\hline 1888 & 0.044698034 & 68 & 0.221940082 & 6792 & 0.039534884 \\
\hline 2954 & 0.0524223 & 78 & 0.231829825 & 7798 & 0.039623646 \\
\hline 3953 & 0.052316998 & 89 & 0.246174407 & 9892 & 0.04329842 \\
\hline 4319 & 0.052316998 & 99 & 0.258024279 & 11566 & 0.047061956 \\
\hline 4986 & 0.044698034 & 109 & 0.270408731 & 13655 & 0.051571099 \\
\hline 6005 & 0.044803335 & 119 & 0.270408731 & 14767 & 0.05796201 \\
\hline 7094 & 0.044701274 & 129 & 0.274863571 & 15812 & 0.066252441 \\
\hline 8061 & 0.052955287 & 139 & 0.282258603 & 16850 & 0.075661282 \\
\hline 9060 & 0.044701274 & 149 & 0.293662991 & 17951 & 0.085070122 \\
\hline 10041 & 0.044913497 & 174 & 0.30310725 & 18945 & 0.098988106 \\
\hline 10952 & 0.04441129 & 199 & 0.322530349 & 19507 & 0.110651518 \\
\hline 12011 & 0.052135556 & 224 & 0.332330995 & 20682 & 0.126841825 \\
\hline 12325 & 0.052240857 & 250 & 0.342755318 & & \\
\hline 12985 & 0.051924953 & 275 & 0.342755318 & & \\
\hline 13965 & 0.061342142 & 300 & 0.360663771 & & \\
\hline 14350 & 0.061342142 & 324 & 0.360663771 & & \\
\hline 14984 & 0.052240857 & 348 & 0.36984074 & & \\
\hline 15261 & 0.05244174 & 374 & 0.378572224 & & \\
\hline 15525 & 0.060997077 & 399 & 0.394698742 & & \\
\hline 15746 & 0.068635482 & 410 & 0.40164829 & & \\
\hline 15846 & 0.086356905 & 414 & 0.414834614 & & \\
\hline 16078 & 0.108662992 & 415 & 0.441830939 & & \\
\hline 16098 & 0.197524452 & & & & \\
\hline 16158 & 0.249374883 & & & & \\
\hline
\end{tabular}


Data of Cotton et al.[39]

\begin{tabular}{|c|c|c|c|c|c|}
\hline & & $\begin{array}{l}\text { Donor Age } \\
\Delta \sigma(M P a) \\
E_{0}(G P a) \\
N_{f} \\
n \text { (cycle) }\end{array}$ & $\begin{array}{l}53 \\
72 \\
15 \\
2626 \\
D \text { (damage) }\end{array}$ & & \\
\hline 0 & 0 & 1249 & 0.130208333 & 2600 & 0.248697917 \\
\hline 21 & 0.013020833 & 1319 & 0.131510417 & 2610 & 0.317708333 \\
\hline 27 & 0.022135417 & 1404 & 0.109375 & 2621 & 0.329427083 \\
\hline 48 & 0.024739583 & 1479 & 0.11328125 & 2626 & 0.421875 \\
\hline 85 & 0.028645833 & 1511 & 0.1171875 & & \\
\hline 101 & 0.036458333 & 1730 & 0.135416667 & & \\
\hline 181 & 0.041666667 & 1751 & 0.16015625 & & \\
\hline 203 & 0.049479167 & 1868 & 0.153645833 & & \\
\hline 230 & 0.059895833 & 2071 & 0.174479167 & & \\
\hline 272 & 0.059895833 & 2077 & 0.157552083 & & \\
\hline 374 & 0.087239583 & 2162 & 0.165364583 & & \\
\hline 464 & 0.088541667 & 2242 & 0.1875 & & \\
\hline 518 & 0.075520833 & 2279 & 0.192708333 & & \\
\hline 582 & 0.092447917 & 2306 & 0.18359375 & & \\
\hline 678 & 0.076822917 & 2343 & 0.1953125 & & \\
\hline 731 & 0.098958333 & 2391 & 0.192708333 & & \\
\hline 806 & 0.102864583 & 2445 & 0.196614583 & & \\
\hline 945 & 0.096354167 & 2493 & 0.200520833 & & \\
\hline 1025 & 0.11328125 & 2536 & 0.221354167 & & \\
\hline 1046 & 0.096354167 & 2552 & 0.20703125 & & \\
\hline 1116 & 0.127604167 & 2557 & 0.21875 & & \\
\hline 1238 & 0.111979167 & 2589 & 0.235677083 & & \\
\hline
\end{tabular}




\section{References}

[1] Hollister, S.J., 2001, “Bone Structure”, BME/ME 456 Biomechanics, University of Michigan.

[2] Lian, J.B., and Stein, G.S., 1996, “Osteoblast biology”, pp. 23-59.

[3] Hayes, W.C., 1986, “Bone mechanics: From tissue mechanical properties to an assessment of structural behaviour:", In Y.C. Fung (Ed.), Frontiers in Biomechanics, 196-209. New York: Schmid-Schonbein.

[4] Lakes, R.S., 1993, “Materials with structural hierarchy".

[5] Martin, R.B., Burr, D.B., 1998, "Skeletal Tissue Mechanics”, Springer Verlag, New York.

[6] Schaffler, M.B., Jepsen, K.J., 2000, "Fatigue and repair in bone", International Journal of Fatigue 22 (10), pp. 839-846.

[7] Ascenzi, A., Bonucci, E., 1970, "The mechanical properties of the osteon in relation to its structural organisation", Chemistry and molecular biology of the intercellular matrix, Academic Press, New York.

[8] Martin, R.B., Gibson, V.A., Stover, S.M., Gibeling, J.C., Griffin, L.V., 1996, “Osteonal structure in the equine third metacarpus", Bone 19, 165-171.

[9] Weiner, S., Traub, W., 1992, "Bone structure: from angstroms to microns", The FASEB journal, 6:879-885.

[10] Forwood, M.R., \& Turner, C.H., 1995. "Skeletal adaptations to mechanical usage.", Bone, 17, $197 \mathrm{~s}-205 \mathrm{~s}$.

[11] Katz, J. L. 2000, “Mechanics of Hard Tissue” The Biomedical Engineering Handbook: Second Edition. Ed. Joseph D. Bronzino, Boca Raton: CRC Press LLC.

[12] Currey, J.D., 2002. “Bones, Structure and Mechanics”, Princeton University Press, Princeton, NJ.

[13] Reilly, D.T, Burstein, A.H, 1975, "The elastic and ultimate properties of compact bone tissue.", Journal of biomechanics 8:393-405.

[14] Carter, D.R., Caler, W.E., 1983, "Cycle-dependent and time-dependent bone fracture with repeated loading.", Journal of biomechanical engineering 105:166-70. 
[15] Burstein, A.H., Currey, J.D., Frankel, V.H., Railly. D.T., 1972, “The ultimate properties of bone tissue: the effects of yielding" Journal of biomechanics 5:35-44.

[16] Carter, D.R., Hayes, W.C. and Schurman, D.J., 1976. "Fatigue life of compact bone -II. Effect of microstructure and density." J. Biomechanics 9, pp. 211-218.

[17] Riggs, C.M., Lanyan, L.E., Boyde, A., 1993, "Functional association between collagen fibre orientation and locomotor strain direction in cortical bone of equine radius.", Anatomy and embryology 187:231-38.

[18] Kotha, S.P., Guzelsu, N., 2007, “Tensile behaviour of cortical bone: Dependence of organic matrix material properties on bone mineral content", Journal of Biomechanics 40-1 pp. 36-45

[19] Currey, J.D., 1962, “Stress concentrations in bone.”, Quarterly journal of microscopical science 103:111-33.

[20] O’Brien, F.J., Taylor, D., Clive Lee, T., 2003, “Microcrack accumulation at different intervals during fatigue testing of compact bone", Journal of Biomechanics 36 973-980.

[21] Currey, J.H, Brear, K., 1974, “Tensile yield in bone.”, Calcified tissue research 15:173-79.

[22] Burr, D.B., Turner, C.H., Naick , P., 1998. "Does microdamage accumulation affect the mechanical properties of bone?", Journal of Biomechanics 31, pp. 337-345.

[23] Railly, G.C., 2001. “Observations of microdamage around osteocyte lacunae in bone.”, Journal of biomechanics $33: 1131-34$.

[24] Pattin, C.A., Caler, W.E., Carter, D.R., 1996. “Cyclic mechanical property degradation during fatigue loading of cortical bone.”, Journal of Biomechanics 29 (1), pp. 69-79.

[25] Zioupos, P., Casinos, A.. 1998. "Cumulative damage and the response of human bone in twostep loading fatigue.", Journal of Biomechanics 31 (9), pp. 825-833.

[26] Hiller, L.P., Stover, S.M., Gibson, V.A., Gibeling, J.C., Prater, C.S., Hazelwood, S.J., Yeh, O.C., Martin, R.B., 2003, “Osteon pullout in the equine third metacarpal bone: Effects of ex vivo fatigue", Journal of Orthopaedic Research 21 (3), pp. 481-488.

[27] Burr, D.B., Martin, R.B., Schaffler, M.B. and Radin, E.L., 1985. "Bone remodelling in response to in vivo fatigue microdamage.", Journal of Biomechanics 18, pp. 189-200.

[28] Swanson, S.A., Freeman M.A., Day, W.H., 1971, "The fatigue properties of human cortical bone.", Pergamon Press, Med. and Bio. Engineering, 9:23-32.

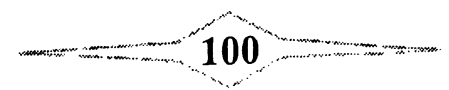


[29] Schaffler, M.B., Choi, K., Milgrom, C., 1995, "Aging and matrix microdamage accumulation in human compact bone.", Bone 17 (6), pp. 521-525.

[30] George, W.T., Vashishth D., 2005, "Damage mechanisms and failure modes of cortical bone under components of physiological loading”, Journal of Orthopaedic Research 23, 1047-1053

[31] Diab, T., Sit, S., Kim, D., Rho, J., Vashishth, D., 2005, “Age-dependent fatigue behaviour of human cortical bone", European Journal of Morphology, 42(1/2): 53 - 59.

[32] Griffin ,L.V., Gibeling, J.C., Martin ,R.B., Gibson ,V.A., Stover ,S.M., 1997, “Model of flexural fatigue damage accumulation for cortical bone", Journal of Orthopaedic Research 15 (4), 607-614.

[33] Ramakrishnan V., Jayaraman N., 1993, "Mechanistically based fatigue-damage evolution model for brittle matrix fibre-reinforced composites", Journal of materials science 28, 55925602.

[34] Maat, G.J.R., Maes, A., Aarents, M.J., Nagelkerke, N.J.D., 2006, “Histological age prediction from the femur in a contemporary Dutch sample”, Journal of Forensic Sciences 51 (2), pp. 230237.

[35] Taylor, D., Lee, T.C., 2003, "Microdamage and mechanical behaviour: Predicting failure and remodelling in compact bone”. Journal of Anatomy, 203 (2), pp. 203-211

[36] Bigley, R.F., Griffin, L.V., Christensen, L., Vandenbosch, R., 2006, “Osteon interfacial strength and histomorphometry of equine cortical bone.", Journal of Biomechanics 39 () 16291640.

[37] Katz, J.L., 1981, “Composite material models for cortical bone. In: Cowin (1981)”, pp. 171-84

[38] Griffin ,L.V., Gibeling, J.C., Martin ,R.B., Gibson ,V.A., Stover ,S.M., 1999, "The effects of testing methods on the flexural fatigue life of human cortical bone", Journal of Biomechanics $32,105-109$.

[39] Cotton, J.R., Winwood, K., Zioupos, P., Taylor, M., 2005. "Damage rate is a predictor of fatigue life and creep strain rate in tensile fatigue of human cortical bone samples", Journal of Biomechanical Engineering 127 (2), pp. 213-219.

[40] Carter, D.R. Hayes, W.C., 1976. "Fatigue life of compact bone -I effects of stress amplitude, temperature and density". Journal of biomechanics Vol. 9, pp. 27-34. 
[41] Taylor, D. and Prendergast, P.J. 1997. “A model for fatigue crack propagation and remodelling in compact bone", Proceedings Of The Institution of Mechanical Engineers Part H - Journal of Engineering in Medicine 211(5), 369-375.

[42] Pidaparti, R.M., Wang, Q.Y., Burr, D.B.; 2001. “Modeling fatigue damage evolution in bone”, Bio-Medical Materials and Engineering 11 (2), pp. 69-78.

[43] Reifsnider, K. 1991, "Fatigue of composite materials", Amsterdam, Elsevier. 